\title{
Nuclear chiral doublet bands data tables
}

\author{
B.W. Xiong \\ State Key Laboratory of Nuclear Physics and Technology, School of Physics, Peking University, Beijing 100871, China. \\ Y.Y. Wang* \\ School of Physics and Nuclear Energy Engineering and International Research Center for Nuclei and Particles in the Cosmos, Beihang \\ University, Beijing 100191, China.
}

\begin{abstract}
Since the prediction of nuclear chirality in 1997, tremendous progresses both theoretically and experimentally have been achieved. Experimentally, 59 chiral doublet bands in 47 chiral nuclei (including 8 nuclei with multiple chiral doublets) $\checkmark$ have been reported in $A \sim 80,100,130$, and 190 mass regions. The spins, parities, energies, ratios of the magnetic dipole transition strengths to the electric quadrupole transition strengths, and related references for these nuclei are compiled and listed in Table 1. For these nuclei with the magnetic dipole transition strengths and the electric quadrupole transition strengths measured, the corresponding results are given in Table 2. A brief discussion is provided after the presentation of energy $E$, energy difference $\Delta E$, energy staggering parameter $S(I)$, rotational frequency $\omega$, kinematic moment of inertia $\mathcal{J}^{(1)}$, dynamic moment of inertia $\mathcal{J}^{(2)}$, and ratio of the magnetic dipole transition strength to the electric quadrupole transition strength $B(M 1) / B(E 2)$ versus spin $I$ in each mass region.
\end{abstract}

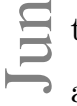

Keywords: Chirality; Chiral doublet bands; Multiple chiral doublets; Spin; Parity; Energy; Electromagnetic transition probability.

*flyyuan@buaa.edu.cn(Y.Y. Wang) 


\section{Contents}

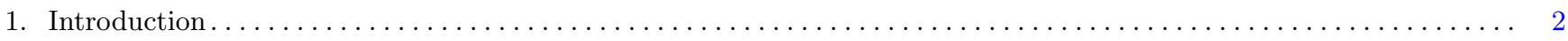

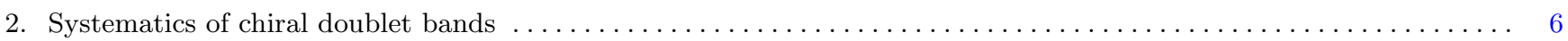

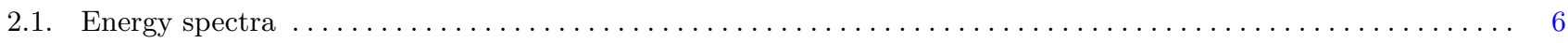

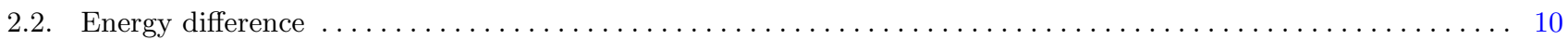

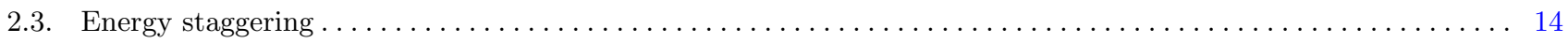

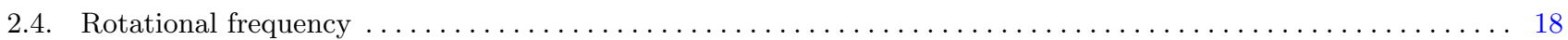

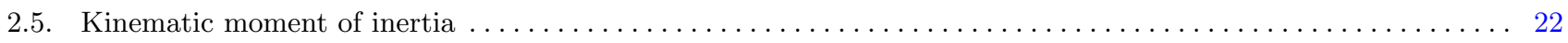

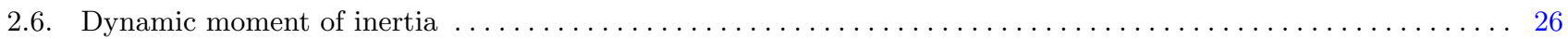

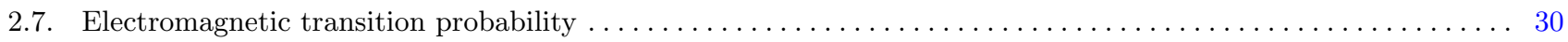

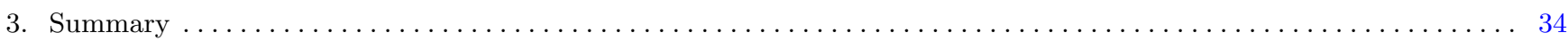

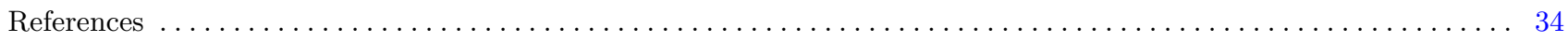

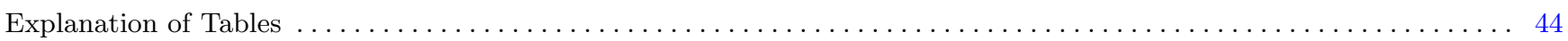

Tables

1. Chiral doublet bands. See page 44 for Explanation of Tables for details. $\ldots \ldots \ldots \ldots \ldots \ldots \ldots \ldots \ldots \ldots \ldots$

2. Chiral doublet bands with $B(M 1)$ and $B(E 2)$ values. See page 44 for Explanation of Tables for details..... 66

\section{Introduction}

Chirality commonly exists in nature, such as the macroscopic spirals of snail shells, the microscopic handedness of certain molecules, and human hands [1]. In geometry, a figure is chiral if it cannot be mapped onto its mirror image by rotations and translations alone. In particle physics, chirality is a dynamic property distinguishing between the parallel and anti-parallel orientations of the intrinsic spin with respect to the momentum of the massless particle. In chemistry, the study of chirality is a very active topic appearing in inorganic, organic, physical, biochemistry, and supramolecular chemistry.

The chirality in nuclear physics was originally suggested by Frauendorf and Meng in 1997 [2]. The physics mechanism of nuclear chirality is illustrated in Fig. 1. For a rotational nucleus with specific triaxial deformation, the collective angular momentum favors alignment along the intermediate axis, which in this case has the largest moment of inertia, while the angular momentum vectors of the valence particles (holes) favor alignment along the nuclear short (long) axis. The three mutually perpendicular angular momenta can be arranged to form two systems with opposite chirality, namely left- and right-handedness. These two systems are transformed into each other by the chiral operator which combines time reversal and spatial rotation of $180^{\circ}, \chi=\mathcal{T} \mathcal{R}(\pi)$. The spontaneous breaking of chiral symmetry thus happens in the body-fixed reference frame. In the laboratory reference frame, with the restoration of chiral symmetry due to quantum tunneling, the so-called chiral doublet bands, i.e., a pair of $\Delta I=1$ bands (normally near degenerate) with the same parity, are expected to be observed in triaxial nuclei $[2,3]$.

The nuclear chirality, originally suggested in Ref. [2] and vigorously investigated over the past few years from both 

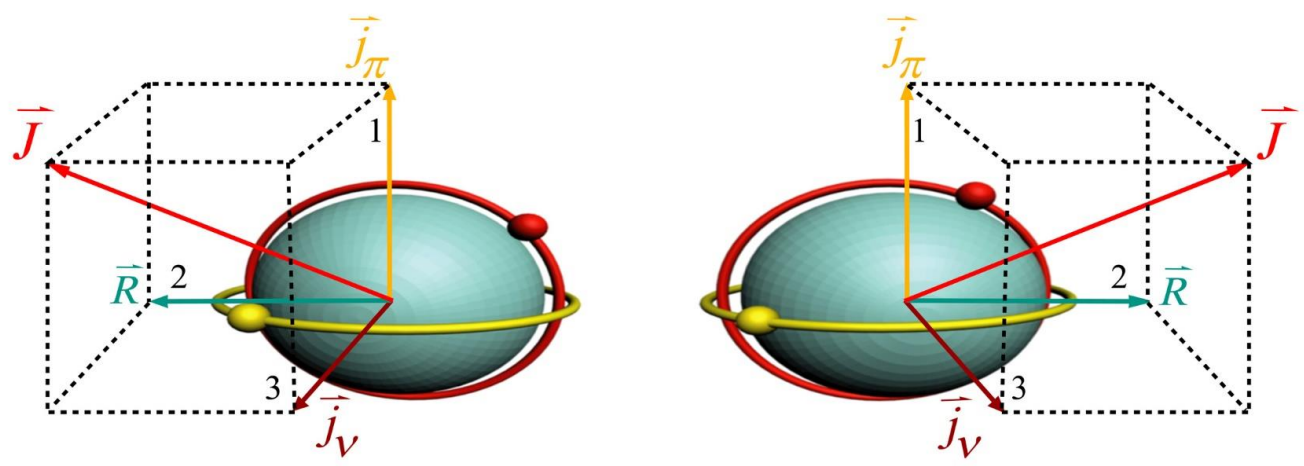

Fig. 1: (Color online) Left- and right-handed chiral systems for a triaxial odd-odd nucleus [3].

the theoretical and experimental standpoint, continues to be the subject of intense discussion. The nuclear chirality has become one of the hot topics in current nuclear physics frontiers, as discussed in recent review articles [3-10].

Theoretically, the nuclear chirality is firstly predicted by particle rotor model (PRM) and tilted axis cranking (TAC) approach in a single- $j$ shell [2]. Later on, various approaches have been developed to describe the nuclear chiral doublet bands.

Depending on the number of valence nucleons and the orbits occupied, 1-particle-1-hole PRM [2, 11-15], 2-quasiparticles PRM [16-19], and $n$-particle- $n$-hole PRM [20-22] have been developed. The varieties of PRM include core quasiparticle coupling model [23-25], interacting boson fermion-fermion model [26-29], the generalized coherent state model [30], the angular momentum projection method [31], and pair truncated shell model [32, 33].

The TAC adopted in Ref. [2] is based on a single- $j$ mean field. Combining the spherical Woods-Saxon single-particle energies and the deformed part of the Nilsson potential, chiral rotation has been studied by the Strutinsky shell correction TAC method [34]. More microscopically, TAC based on covariant density functional theory (CDFT) [35, 36] has been introduced and applied to the studies of chirality. The self-consistent Skyrme Hartree-Fock cranking model has also been developed [37].

To go beyond mean field approximation to describe the chiral partners, one can incorporate the quantum correlations by means of random phase approximation $[38,39]$ or collective Hamiltonian $[40,41]$. By taking into account the quantum fluctuation along the collective degree of freedom, the collective Hamiltonian goes beyond the mean field approximation and restores the broken symmetry.

The attempts to understand the chiral doublet bands by the projected shell model (PSM) [42] have been performed in Ref. [43]. Although the observed energy spectra and transitions have been well reproduced in PSM, it is a big challenge to examine the chiral geometry of angular momentum due to the complication that the projected basis is defined in the laboratory frame and forms a nonorthogonal set. Recently, the chiral geometry of the angular momentum is investigated within the framework of PSM. The geometry of the angular momentum is analyzed in terms of the distributions of its components on the three intrinsic axes $(K$ plot) as well as the distributions of its tilted angles in the intrinsic frame (azimuthal plot) [44, 45].

The PRM is a quantal model consisting of the collective rotation and the intrinsic single-particle motions, the energy splitting and quantum tunneling between the doublet bands can be obtained directly. The rigid rotor with quadrupole 
deformation parameters $\beta$ and $\gamma$ is assumed [3].

Starting from an effective nucleon-nucleon interaction with Lorentz invariance, the CDFT naturally includes the spinorbit coupling and has achieved great successes in describing many nuclear phenomena in stable and exotic nuclei of the whole nuclear chart [1, 46-49]. It is interesting to search for nuclei with triaxial deformation and configurations having not only one particle and one hole but also several particles and several holes suitable for chirality in CDFT in Ref. [50], the adiabatic and configuration-fixed constrained triaxial CDFT approaches are used to investigate the triaxial shape coexistence and possible chiral doublet bands. A new phenomenon, the existence of multiple chiral doublets (M $\chi \mathrm{D})$, i.e., more than one pair of chiral doublet bands in one single nucleus, is suggested for ${ }^{106} \mathrm{Rh}$. This prediction remains with the time-odd fields included [51], and also holds true for other rhodium isotopes [52].

The first experimental evidence for $\mathrm{M} \chi \mathrm{D}$ is reported in ${ }^{133} \mathrm{Ce}$ in 2013 [53]. Later, a novel type of $\mathrm{M} \chi \mathrm{D}$ with the same configuration is reported in ${ }^{103} \mathrm{Rh}$ [54], which shows that chiral geometry can be robust against the intrinsic excitation. Then, two pairs of positive- and negative-parity doublet bands together with eight strong electric dipole transitions linking their yrast positive- and negative-parity bands, are identified in ${ }^{78} \mathrm{Br}$. This observation reports the first example of chiral geometry in octupole soft nuclei and indicates that nuclear chirality can be robust against the octupole correlations [55]. Recently, five pairs of nearly degenerate rotational bands were identified in ${ }^{136} \mathrm{Nd}$ [56].

It should be mentioned that two pairs of chiral doublet bands in ${ }^{105} \mathrm{Rh}$ have been independently observed in Refs. $[57,58]$, which have been confirmed to be $\mathrm{M} \chi \mathrm{D}$ by adiabatic and configuration-fixed constrained CDFT calculations [59]. Similarly, one pair of chiral doublet bands in ${ }^{107} \mathrm{Ag}$ observed in Ref. [60], which together with nearly degenerate partner bands (though the difference in spin alignment in Fig. 16 needs further clarification) [61], has been claimed to show evidence of $\mathrm{M} \chi \mathrm{D}$ [62]. Similarly, for ${ }^{138} \mathrm{Nd}$, one pair of chiral doublet bands has been suggested in Ref. [63], which together with another pair of partner bands, could be a new candidate of $\mathrm{M} \chi \mathrm{D}[30]$.

Generally speaking, for the description of chiral rotations, three dimensional tilted axis cranking CDFT (3D TACCDFT) is needed. The 3D TAC-CDFT is firstly developed in Ref. [35]. However, because of its numerical complexity, so far, it has been applied only for the magnetic rotation in ${ }^{84} \mathrm{Rb}$. Focusing on the magnetic rotation bands, in 2008 , a completely new computer code for the self-consistent 2D TAC-CDFT has been established [64]. It is based on the non-linear meson-exchange models and includes considerable improvements allowing systematic investigations. Based on a point-coupling interaction, the 2D TAC-CDFT is developed to investigated the magnetic rotation bands [65, 66], antimagnetic rotation bands [67, 68], transitions of nuclear spin orientation [69, 70], and linear alpha cluster bands [71], and demonstrates high predictive power [6,36]. Recently, the first applications of the 3D TAC-CDFT for nuclear chirality is reported in ${ }^{106} \mathrm{Rh}[72]$ and ${ }^{136} \mathrm{Nd}$ [56], respectively.

Originally the observation of two almost degenerate $\Delta I=1$ rotational bands is considered as the fingerprint of chiral doublet bands [2]. With the improvement of experimental techniques, the lifetime measurements for chiral doublet bands become possible. According to the selection rule for the electromagnetic transition in ideal case, there occurs an alternation of stronger and weaker $M 1$ transitions with spin over the degenerate spin range of chiral doublet bands, which can manifest as $B(M 1) / B(E 2)$ staggering as a function of spin $I$. These characteristics are suggested as electromagnetic transition fingerprints of chiral geometry in Ref. [12]. It is pointed out that in ideal chiral doublet bands, the corresponding properties such as the identical or similarity in energies, spin alignments, and electromagnetic transition probabilities, the spin independence of the energy staggering parameters $S(I)$, and the staggering of $B(M 1) / B(E 2)$ are 
summarized as fingerprints of ideal chiral doublet bands [73].

During the last two decades, lots of experimental efforts have been devoted to search for nuclear chirality. Up to now, 59 chiral doublet bands in 47 chiral nuclei (including $8 \mathrm{M} \chi \mathrm{D}$ nuclei) have been reported in the $A \sim 80$ [55, 74], $100[54,57,58,60,75-85], 130$ [38, 53, 56, 63, 86-105], and 190 [106-109] mass regions. The distribution of the observed chiral nuclei in the nuclear chart is given in Fig. 2.

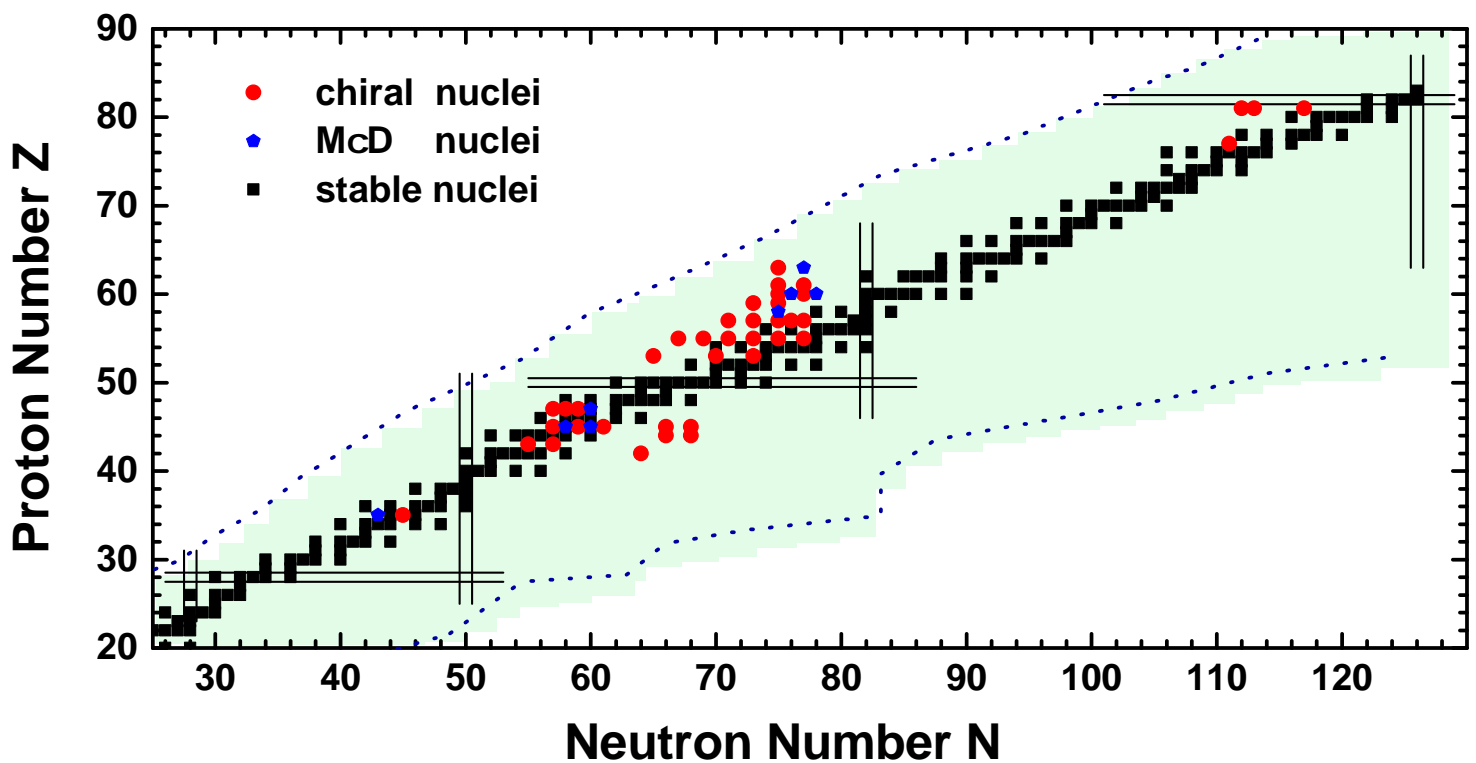

Fig. 2: (Color online) The nuclides with chiral doublet bands (red circles) and $\mathrm{M} \chi \mathrm{D}$ (blue pentagons) observed in the nuclear chart. The black squares represent stable nuclides.

It should be pointed out that the lifetime measurements which are essential to extract the absolute electromagnetic transition probabilities are still rare for the chiral nuclei candidates.

In order to promote the study of chiral symmetry in atomic nuclei, the compilations of the data for chiral doublet bands are highly demanded. This is the purpose of the present paper.

In section 2, the figures are presented for energy $E$, energy difference $\Delta E$, energy staggering parameter $S(I)$, rotational frequency $\omega$, kinematic moment of inertia $\mathcal{J}^{(1)}$, dynamic moment of inertia $\mathcal{J}^{(2)}$, and ratio of the magnetic dipole transition strength to the electric quadrupole transition strength $B(M 1) / B(E 2)$ versus spin $I$ in each mass region. The explanation of the tables for chiral doublet bands is followed. Finally, a brief summary is given. 


\section{Systematics of chiral doublet bands}

\subsection{Energy spectra}

The energy spectra for all chiral doublet bands in $A \sim 80$, 100, 130, and 190 mass regions are given in Fig. 3-6, respectively. For $\mathrm{M} \chi \mathrm{D}$ in ${ }^{78} \mathrm{Br},{ }^{105} \mathrm{Rh},{ }^{107} \mathrm{Ag},{ }^{133} \mathrm{Ce},{ }^{138} \mathrm{Nd}$, and ${ }^{140} \mathrm{Eu}$, the excited chiral doublet bands are shifted by $1.5 \mathrm{MeV}$. For $\mathrm{M} \chi \mathrm{D}$ in ${ }^{103} \mathrm{Rh}$, there are three pairs of chiral doublet bands, one pair of excited bands are shifted by 1.5 $\mathrm{MeV}$, and another are shifted by $3 \mathrm{MeV}$. As for $\mathrm{M} \chi \mathrm{D}$ in ${ }^{136} \mathrm{Nd}$, there are five pairs of chiral doublet bands, the two lowest ones are shift by $-3.0 \mathrm{MeV}$ and $-1.5 \mathrm{MeV}$, respectively, and the next two higher ones are shift by $1.5 \mathrm{MeV}$ and $3.0 \mathrm{MeV}$, respectively.

The fingerprint for chiral doublet bands in energy, i.e., two almost degenerate $\Delta I=1$ rotational bands, is demonstrated in the figures.

The nuclear chirality occurs at the lowest spin $4 \hbar$ in ${ }^{106} \mathrm{Mo}$ and ${ }^{110} \mathrm{Ru}$, and the highest spin $29 \hbar$ in ${ }^{136} \mathrm{Nd}$. The bands in ${ }^{106} \mathrm{Mo}$ and ${ }^{110} \mathrm{Ru}$ are interpreted as soft chiral vibration $[78,110,111]$. In ${ }^{136} \mathrm{Nd}$, the chiral rotations were interpreted by TAC-CDFT with the assigned configurations [56].

Generally, the energies for the partners are close to each other and tend to be almost completely degenerate at high spins. There are several exceptions: for nuclei ${ }^{112} \mathrm{Ru},{ }^{105} \mathrm{Rh},{ }^{107} \mathrm{Ag},{ }^{136} \mathrm{Nd},{ }^{137} \mathrm{Nd},{ }^{140} \mathrm{Eu}$, and ${ }^{188} \mathrm{Ir}$, crossings between some partner bands exist.

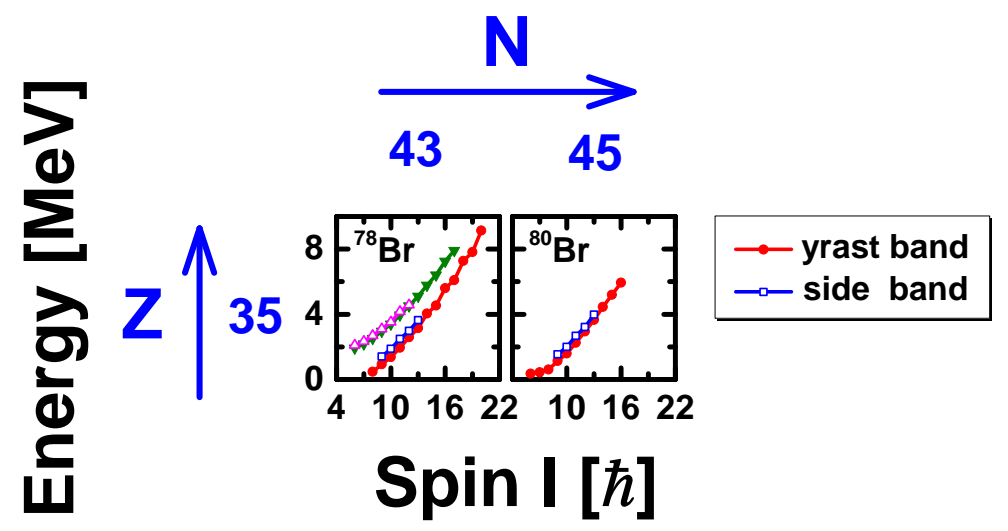

Fig. 3: (Color online) Energies versus spin for chiral doublet bands in $A \sim 80$ mass region. The existence of $\mathrm{M} \chi \mathrm{D}$ is suggested in ${ }^{78} \mathrm{Br}$. The excited chiral doublet bands are shifted by $1.5 \mathrm{MeV}$. 


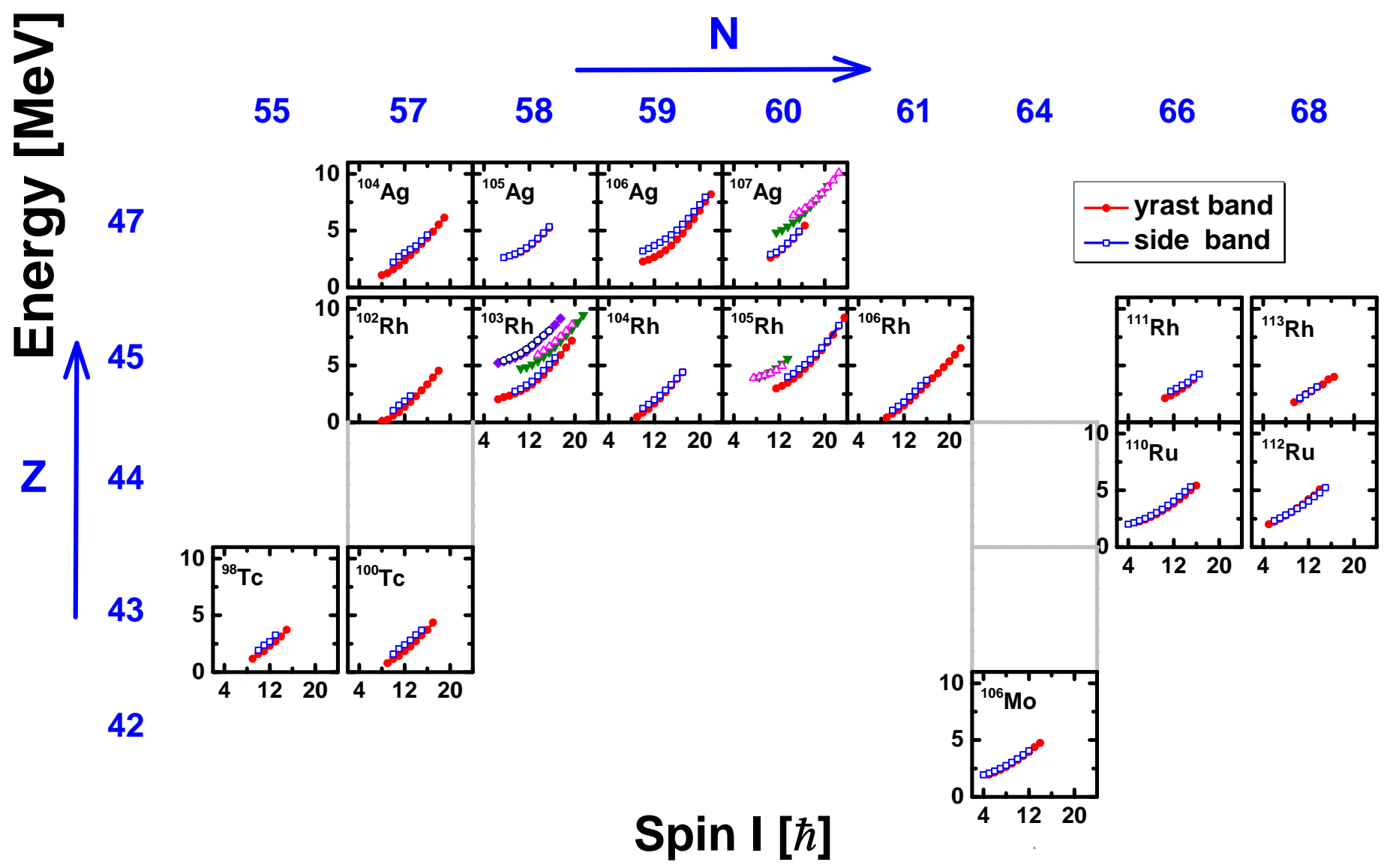

Fig. 4: (Color online) Energies versus spin for chiral doublet bands in $A \sim 100$ mass region. The existence of $\mathrm{M} \chi \mathrm{D}$ are suggested in ${ }^{103} \mathrm{Rh}$, ${ }^{105} \mathrm{Rh}$, and ${ }^{107} \mathrm{Ag}$. One pair of excited bands in ${ }^{103} \mathrm{Rh}$ are shifted by $1.5 \mathrm{MeV}$, and another are shifted by $3 \mathrm{MeV}$. The excited chiral doublet bands in ${ }^{105} \mathrm{Rh}$ and ${ }^{107} \mathrm{Ag}$ are shifted by $1.5 \mathrm{MeV}$. The gray boxes are used to connect the various parts of this figure. 


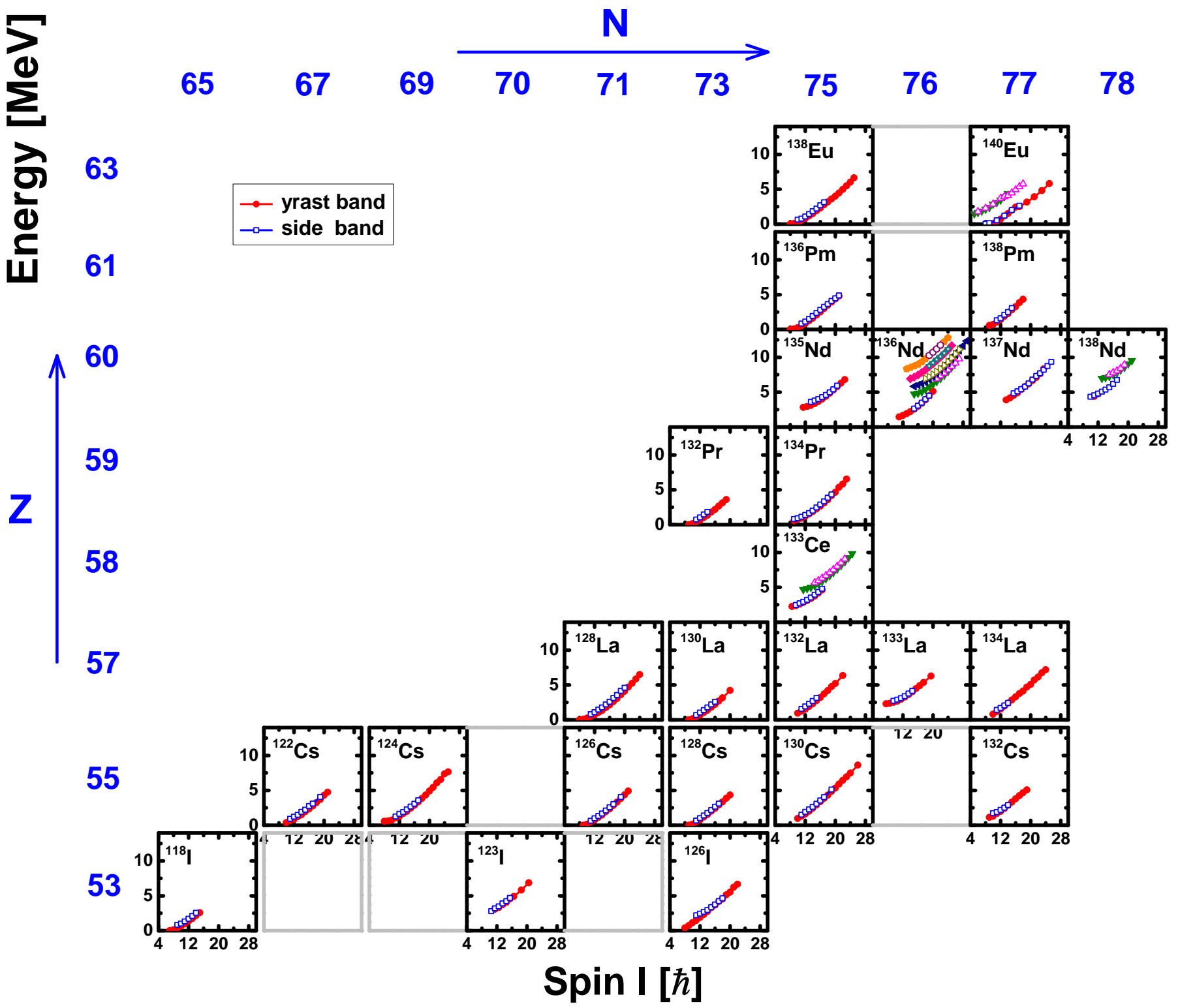

Fig. 5: (Color online) Energies versus spin for chiral doublet bands in $A \sim 130$ mass region. The existences of $\mathrm{M} \chi \mathrm{D}$ are suggested in ${ }^{133} \mathrm{Ce}$, ${ }^{136} \mathrm{Nd},{ }^{138} \mathrm{Nd}$, and ${ }^{140} \mathrm{Eu}$. For ${ }^{136} \mathrm{Nd}$, there are five pairs of chiral doublet bands, the two lowest ones are shift by $-3.0 \mathrm{MeV}$ and $-1.5 \mathrm{MeV}$, respectively, and the next two higher ones are shift by $1.5 \mathrm{MeV}$ and $3.0 \mathrm{MeV}$, respectively. For other $\mathrm{M} \chi \mathrm{D}$, the excited chiral doublet bands are shifted by $1.5 \mathrm{MeV}$. The gray boxes are used to connect the various parts of this figure. 


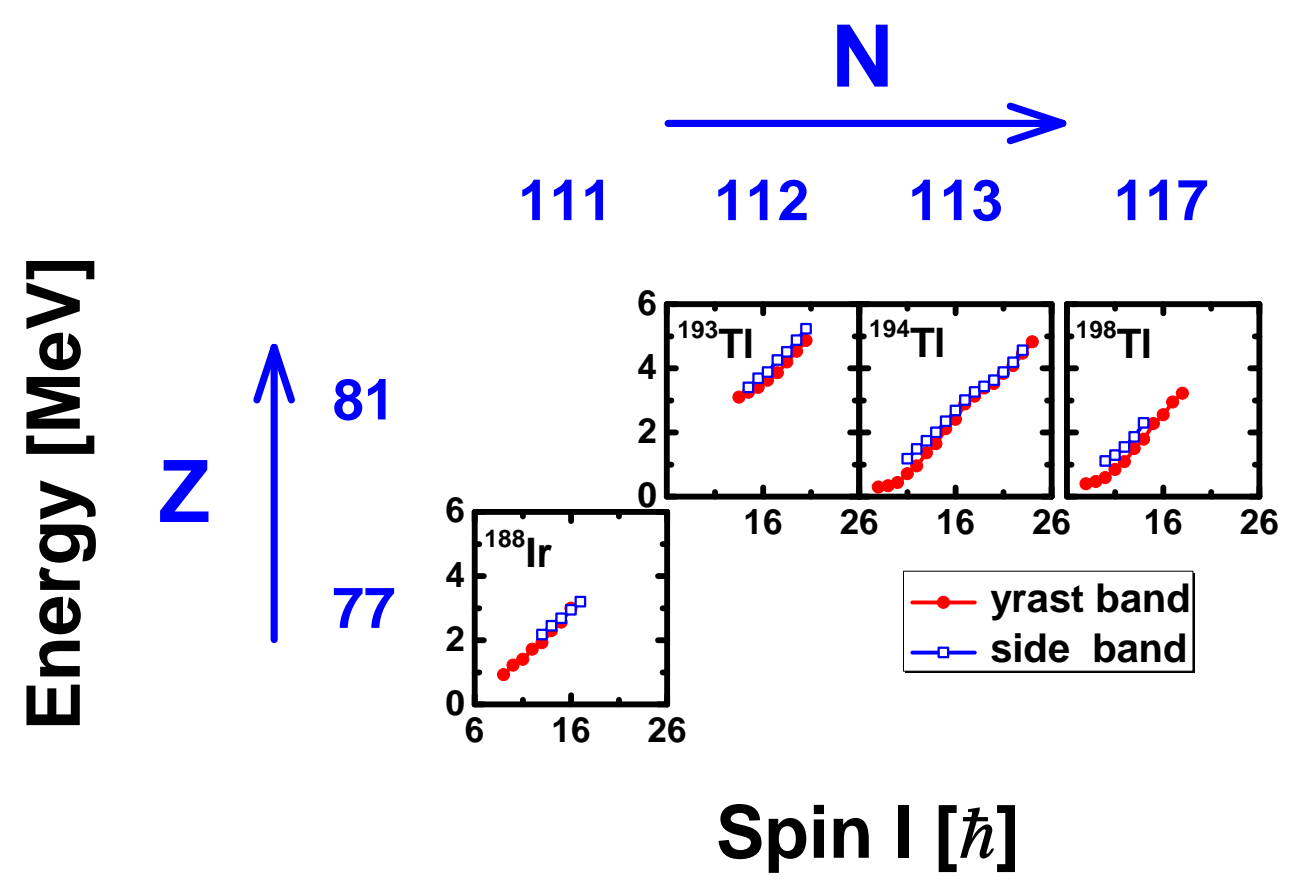

Fig. 6: (Color online) Energies versus spin for chiral doublet bands in $A \sim 190$ mass region. 


\subsection{Energy difference}

The energy differences $\Delta E(I)=E_{\text {side }}(I)-E_{\text {yrast }}(I)$ between yrast band and side band for all chiral doublet bands in $A \sim 80,100,130$, and 190 mass regions are given in Figs. 7- 10, respectively. Although the chiral partner bands have energies close to each other, it is rare to observe a crossing between them. If crossing occurs, carefully examinations for chirality are necessary, as has been done in ${ }^{106} \mathrm{Ag}$ [85].

The energy differences $\Delta E$ are below $600 \mathrm{keV}$ for all chiral doublet bands except for ${ }^{100} \mathrm{Tc},{ }^{102} \mathrm{Rh},{ }^{104} \mathrm{Ag},{ }^{106} \mathrm{Ag}$, ${ }^{107} \mathrm{Ag}$, and ${ }^{126} \mathrm{I}$. Several negative values exist for $\Delta E$ in nuclei ${ }^{112} \mathrm{Ru},{ }^{105} \mathrm{Rh},{ }^{107} \mathrm{Ag},{ }^{136} \mathrm{Nd},{ }^{137} \mathrm{Nd},{ }^{140} \mathrm{Eu}$, and ${ }^{188} \mathrm{Ir}$ which correspond to the crossing between the partner bands in Section 2.1.

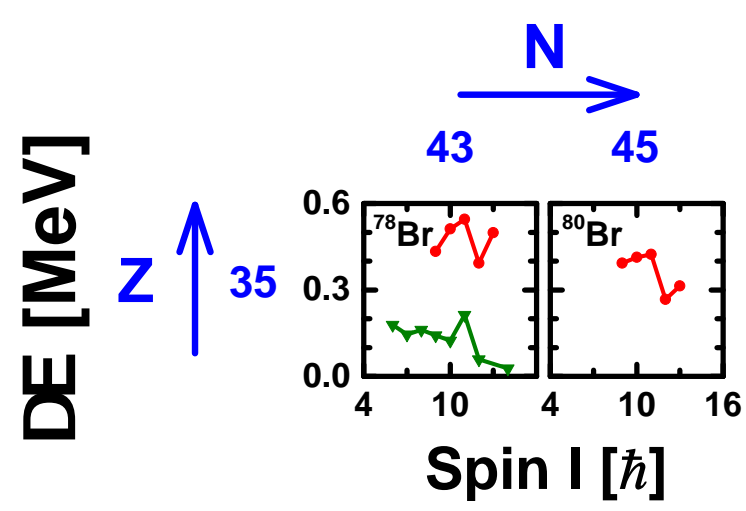

Fig. 7: (Color online) Energy differences between the chiral doublet bands versus spin in $A \sim 80$ mass region. The red and green colors represent the energy differences between the chiral partners and the excited chiral partners, respectively. 


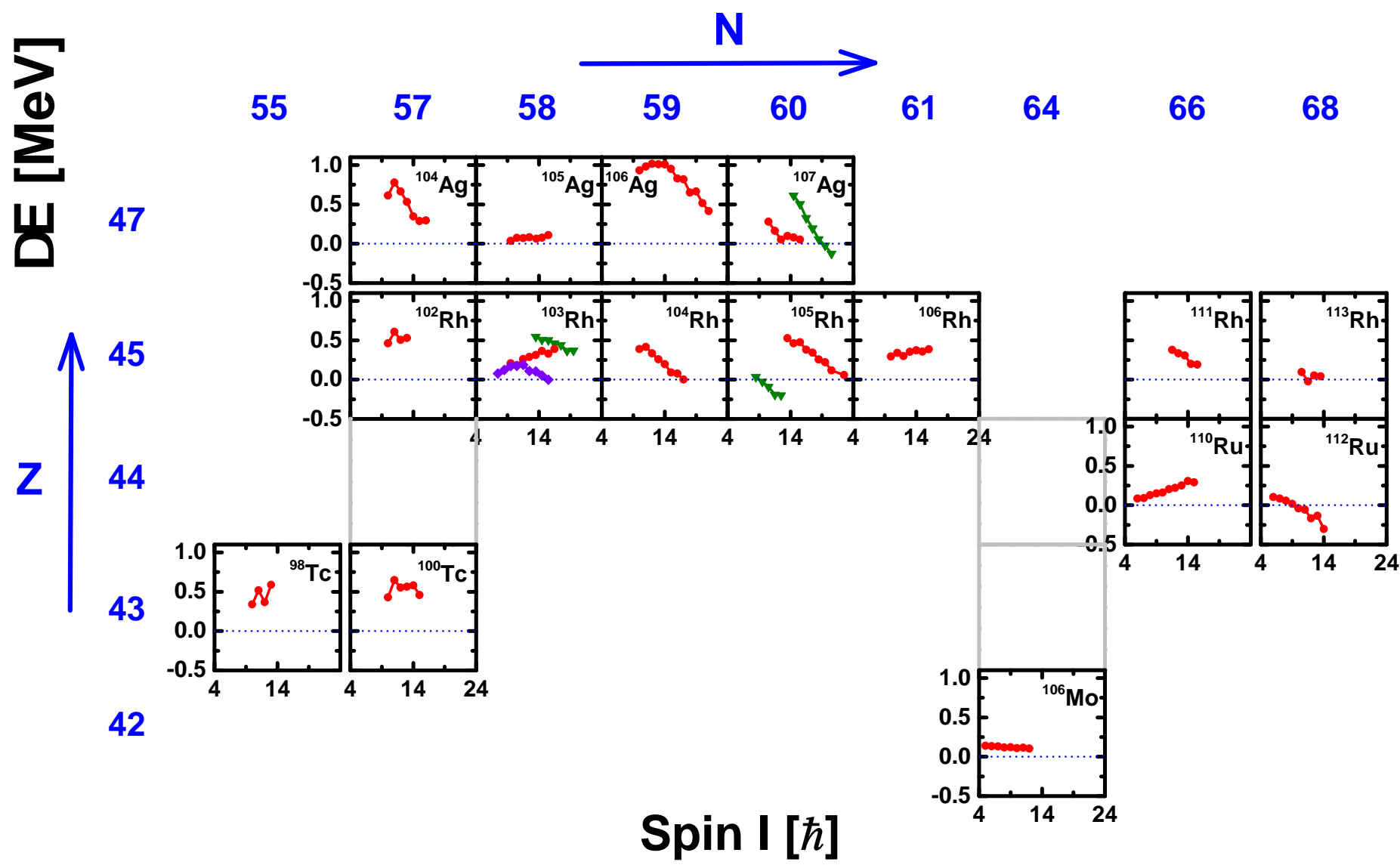

Fig. 8: (Color online) Energy differences between the chiral doublet bands versus spin in $A \sim 100$ mass region. 


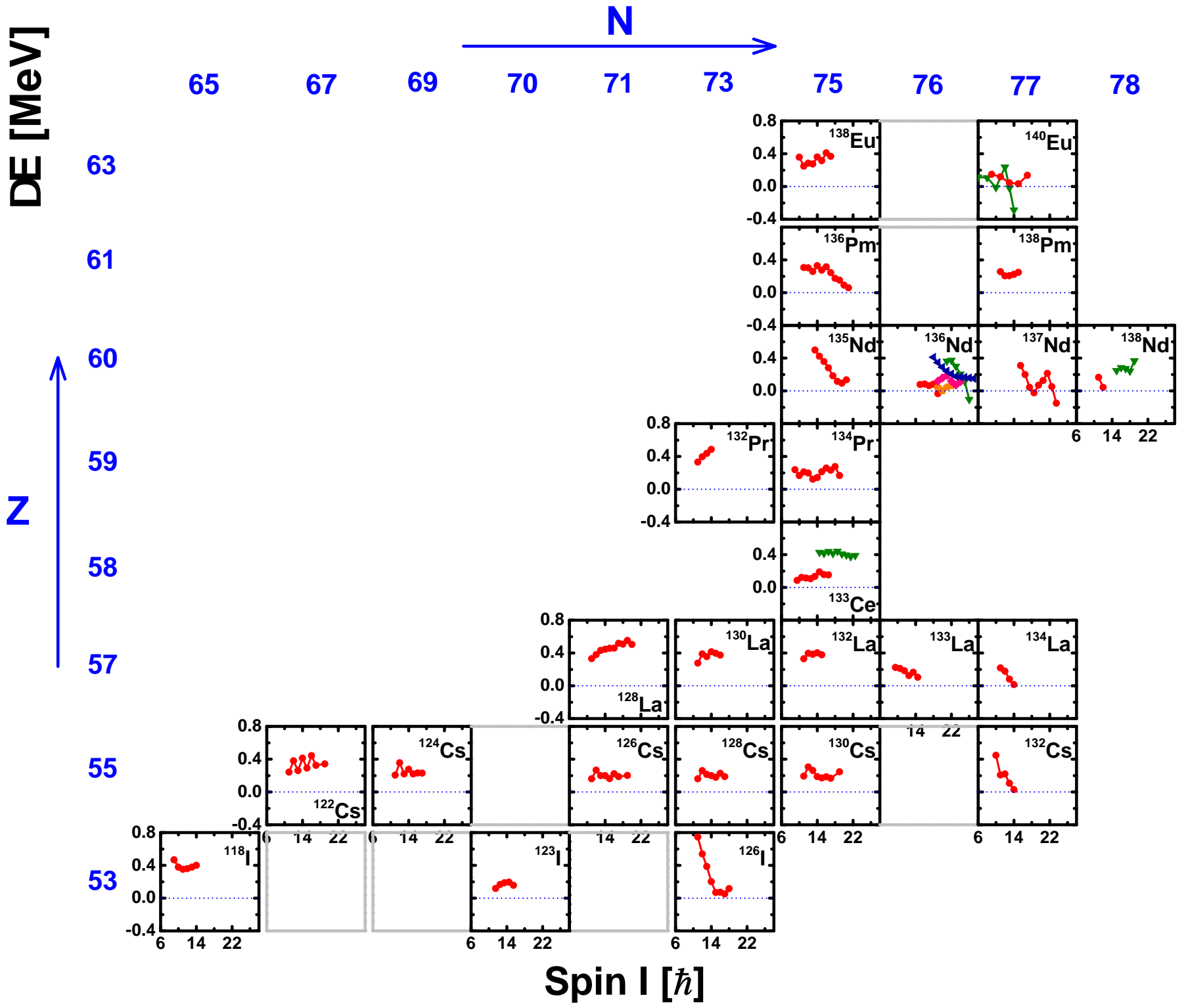

Fig. 9: (Color online) Energy differences between the chiral doublet bands versus spin in $A \sim 130$ mass region. 


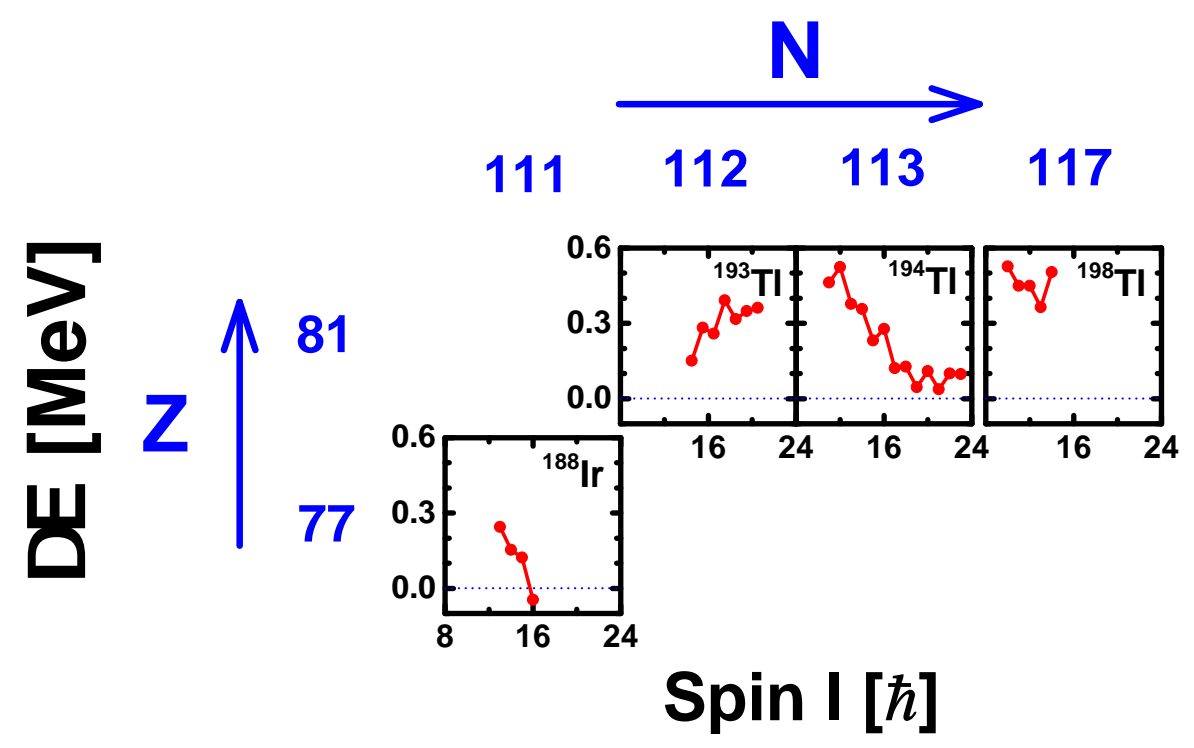

Fig. 10: (Color online) Energy differences between the chiral doublet bands versus spin in $A \sim 190$ mass region. 


\subsection{Energy staggering}

From the energy staggering parameter defined as $S(I)=[E(I)-E(I-1)] / 2 I$, the energy staggering parameters as functions of spin for all chiral doublet bands in $A \sim 80,100,130$, and 190 mass regions are given in Figs. 11-14, respectively.

For the ideal chiral doublet bands, the $S(I)$ values should possess a smooth dependence with spin, and it has been taken as a possible fingerprint.

Normally, the values of $S(I)$ change dramatically at the band head. At certain spin range, they show a smooth dependence with spin. The change of $S(I)$ values is around $20 \mathrm{keV} / \hbar$ in $A \sim 80$ mass region, and decrease to $10 \mathrm{keV} / \hbar$ for most nuclei in $A \sim 100,130$, and 190 mass regions, except for ${ }^{140} \mathrm{Eu},{ }^{194} \mathrm{Tl}$, and ${ }^{198} \mathrm{Tl}$ whose change is around 20 $\mathrm{keV} / \hbar$.

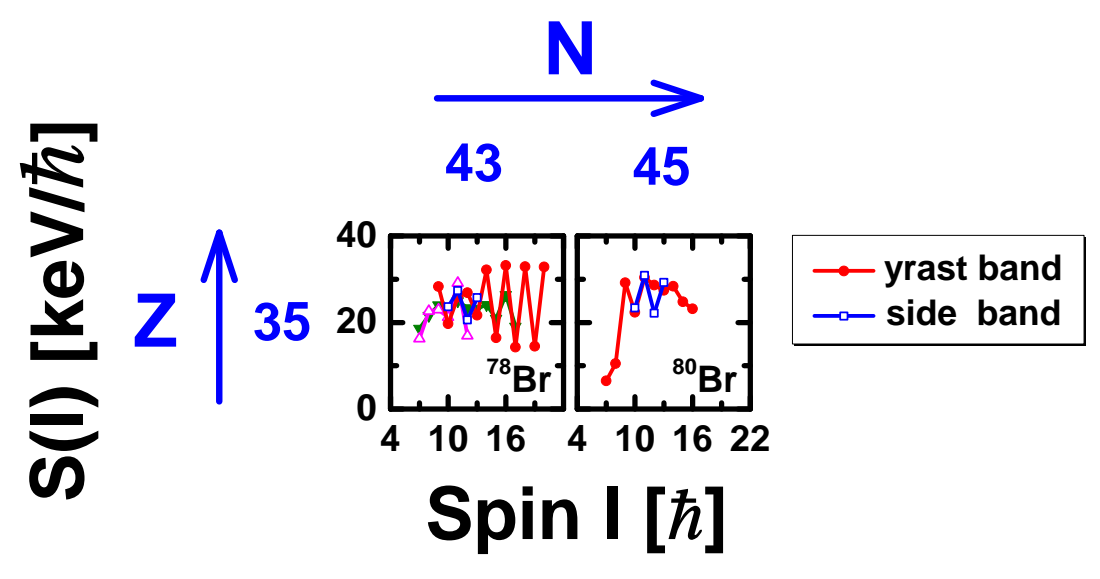

Fig. 11: (Color online) Energy staggering parameters as functions of spin in $A \sim 80$ mass region. 


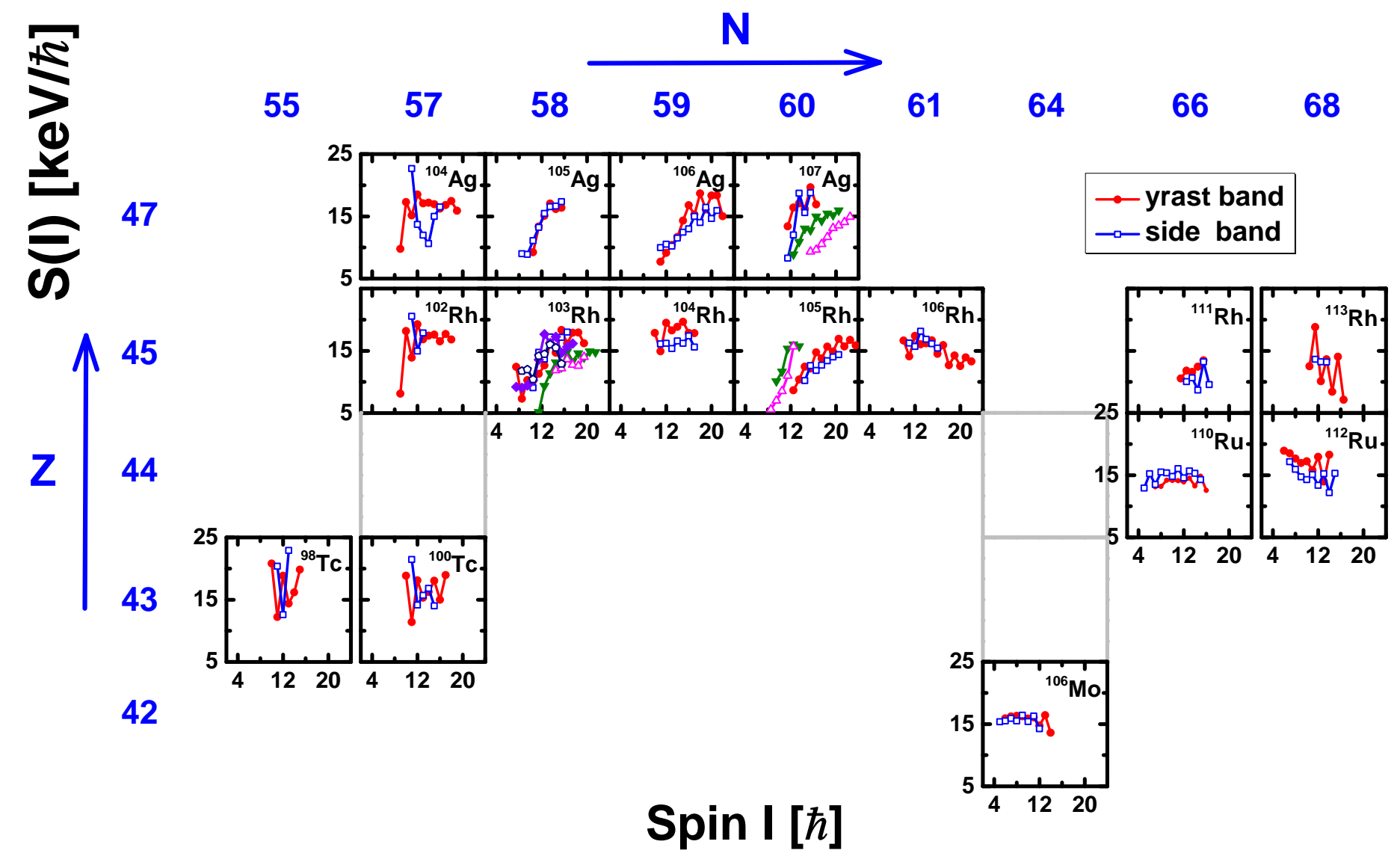

Fig. 12: (Color online) Energy staggering parameters as functions of spin in $A \sim 100$ mass region. 


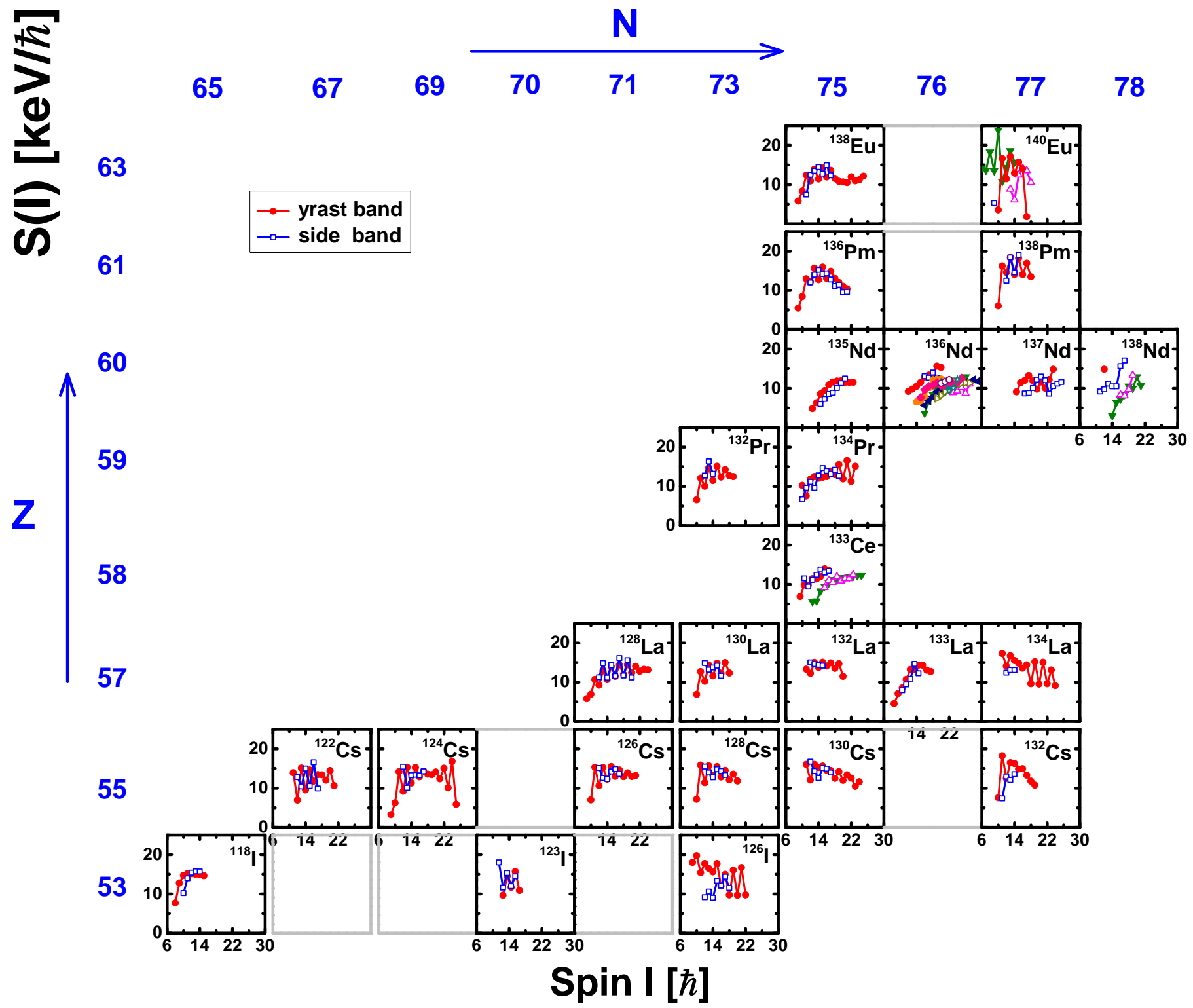

Fig. 13: (Color online) Energy staggering parameters as functions of spin in $A \sim 130$ mass region. 


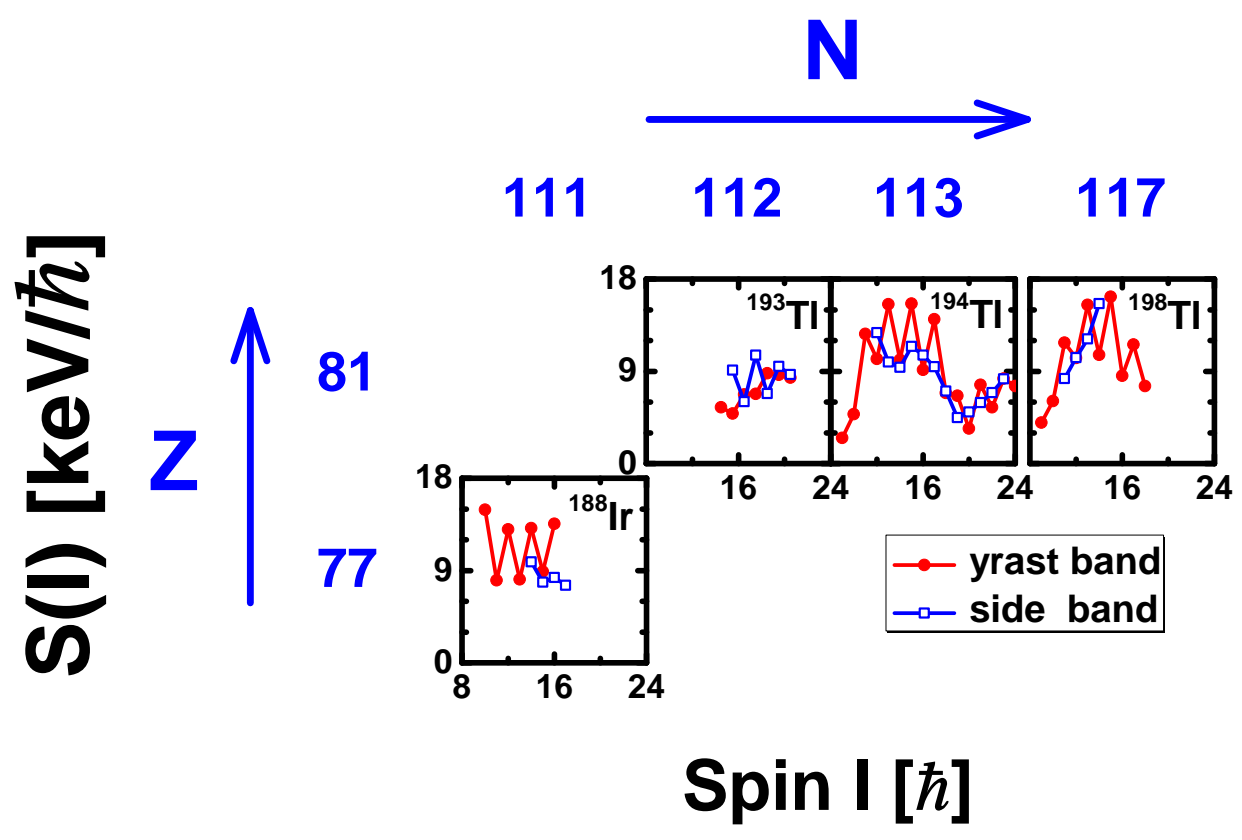

Fig. 14: (Color online) Energy staggering parameters as functions of spin in $A \sim 190$ mass region. 


\subsection{Rotational frequency}

In order to obtain the response of angular momentum alignments to rotation frequencies and examine the similarities of the configurations for chiral partner bands, the $I-\hbar \omega$ relation has been extracted. From the rotational frequency $\hbar \omega$ defined as [112], $\hbar \omega(I)=[E(I+1)-E(I-1)] / 2$, the relations between the spins and the rotational frequencies for all chiral doublet bands in $A \sim 80,100,130$, and 190 mass regions are shown in Figs. 15-18, respectively.

Generally, the $I-\hbar \omega$ relation for yrast band and side band is similar, except for ${ }^{104} \mathrm{Ag},{ }^{107} \mathrm{Ag},{ }^{126} \mathrm{I},{ }^{136} \mathrm{Nd},{ }^{137} \mathrm{Nd}$, ${ }^{188} \mathrm{Ir}$, and ${ }^{193} \mathrm{Tl}$. Possible backbending in some nuclei exists.

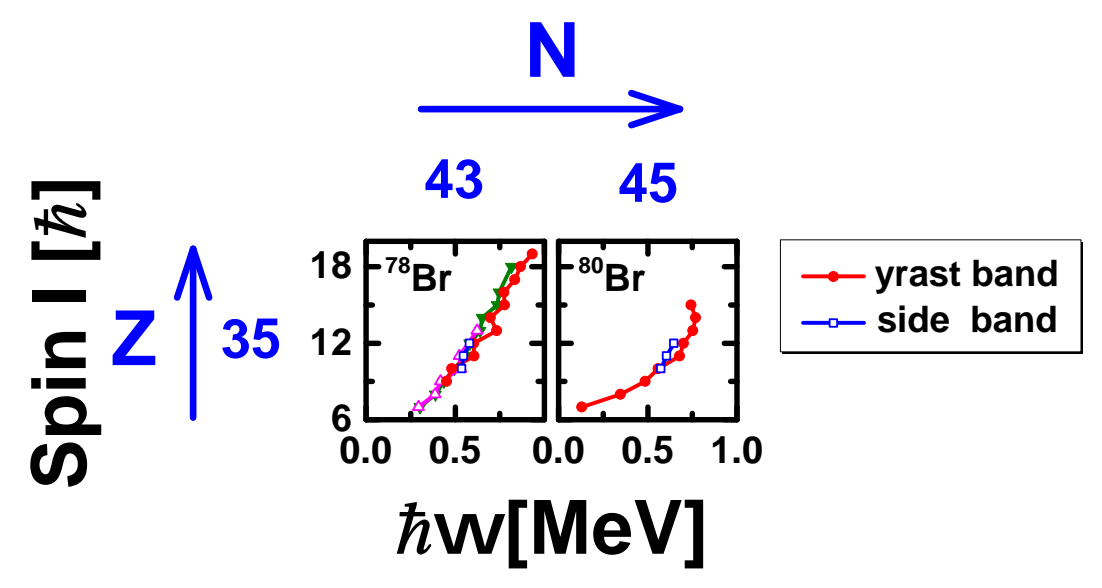

Fig. 15: (Color online) The relation between the spin and the rotational frequency for chiral doublet bands in $A \sim 80$ mass region. 


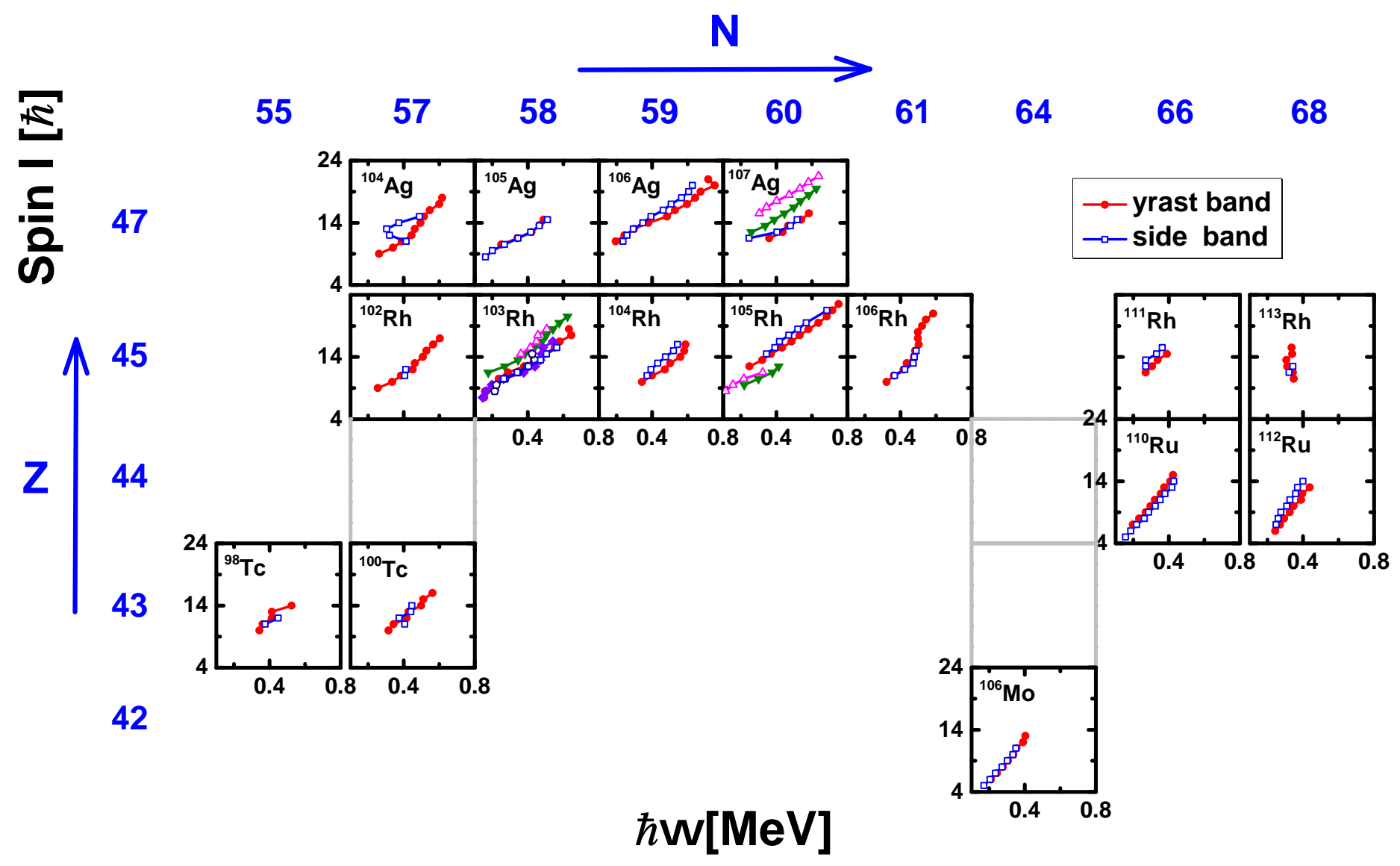

Fig. 16: (Color online) The relation between the spin and the rotational frequency for chiral doublet bands in $A \sim 100$ mass region. 


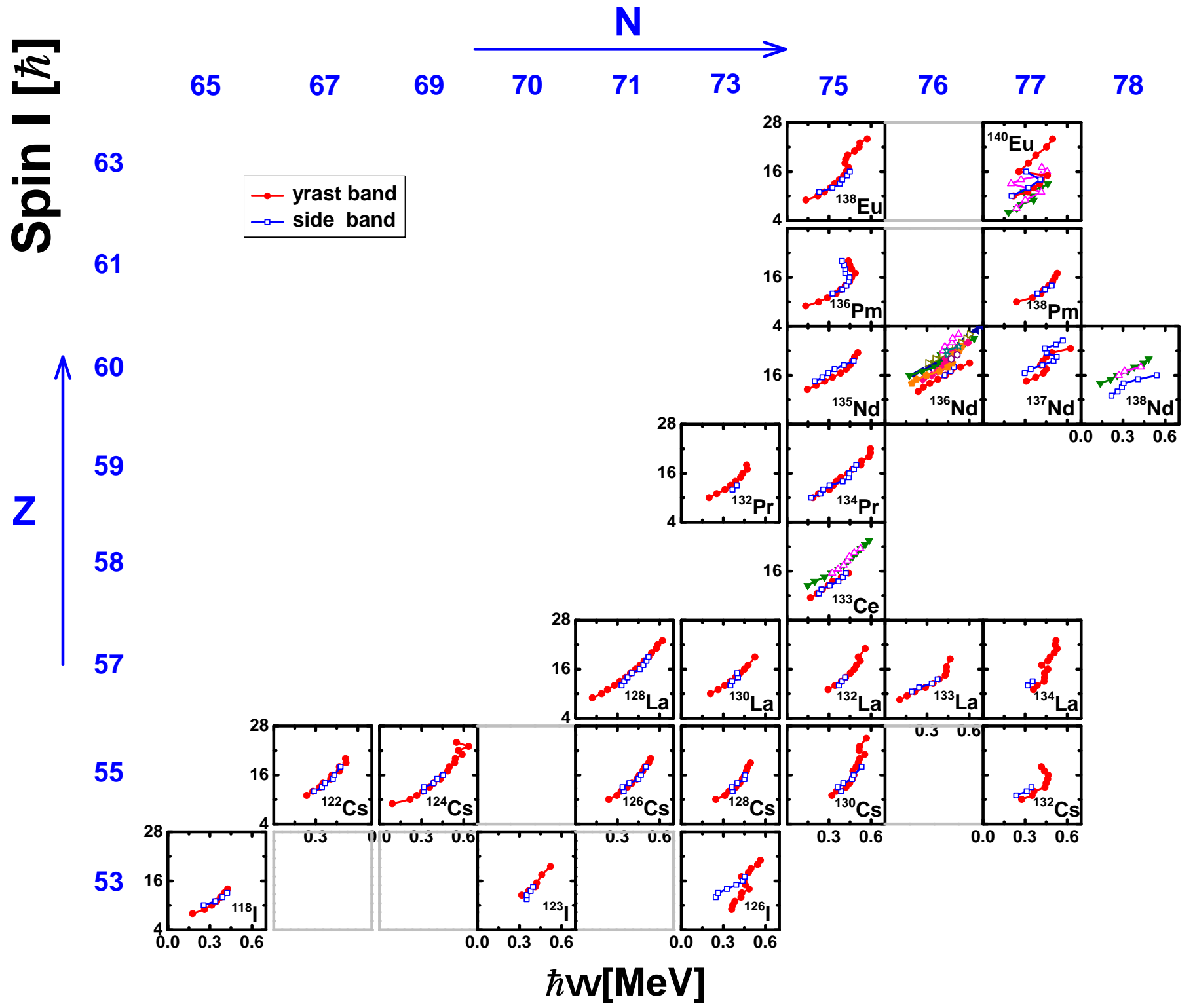

Fig. 17: (Color online) The relation between the spin and the rotational frequency for chiral doublet bands in $A \sim 130$ mass region. 


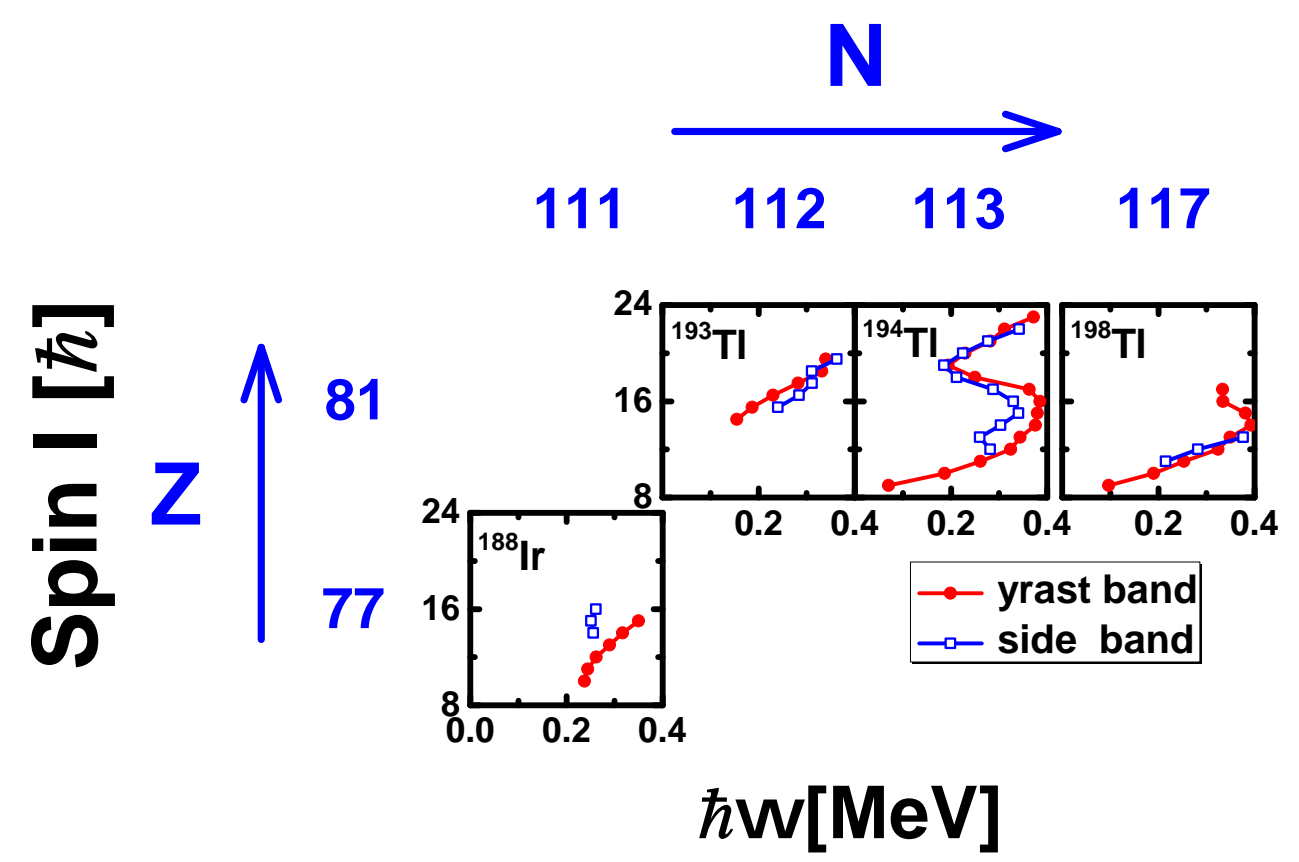

Fig. 18: (Color online) The relation between the spin and the rotational frequency for chiral doublet bands in $A \sim 190$ mass region. 


\subsection{Kinematic moment of inertia}

From the definition $\mathcal{J}^{(1)}(I)=I / \hbar \omega(I)$, the kinematic moments of inertia $\mathcal{J}^{(1)}$ for all chiral doublet bands in $A \sim 80,100,130$, and 190 mass regions are shown in Figs. 19-22, respectively.

Generally, the kinematic moment of inertia for yrast band and side band is similar, except for ${ }^{104} \mathrm{Ag},{ }^{107} \mathrm{Ag},{ }^{126} \mathrm{I}$, ${ }^{136} \mathrm{Nd},{ }^{137} \mathrm{Nd},{ }^{140} \mathrm{Eu}$, and ${ }^{188} \mathrm{Ir}$. Normally, the kinematic moment of inertia remains roughly constant.

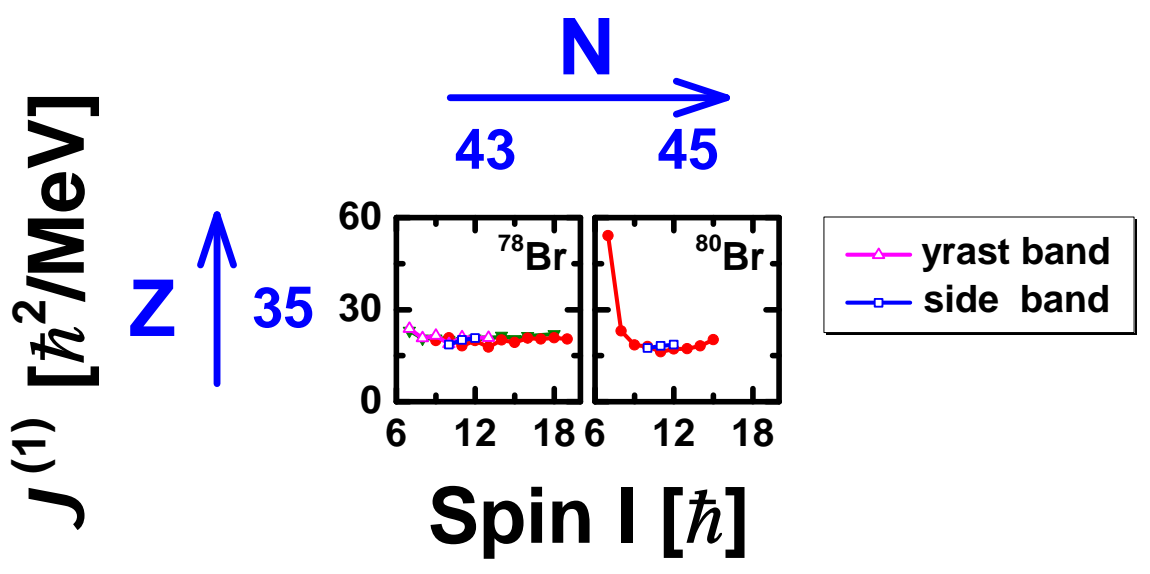

Fig. 19: (Color online) Kinematic moments of inertia versus spin for chiral doublet bands in $A \sim 80$ mass region. 


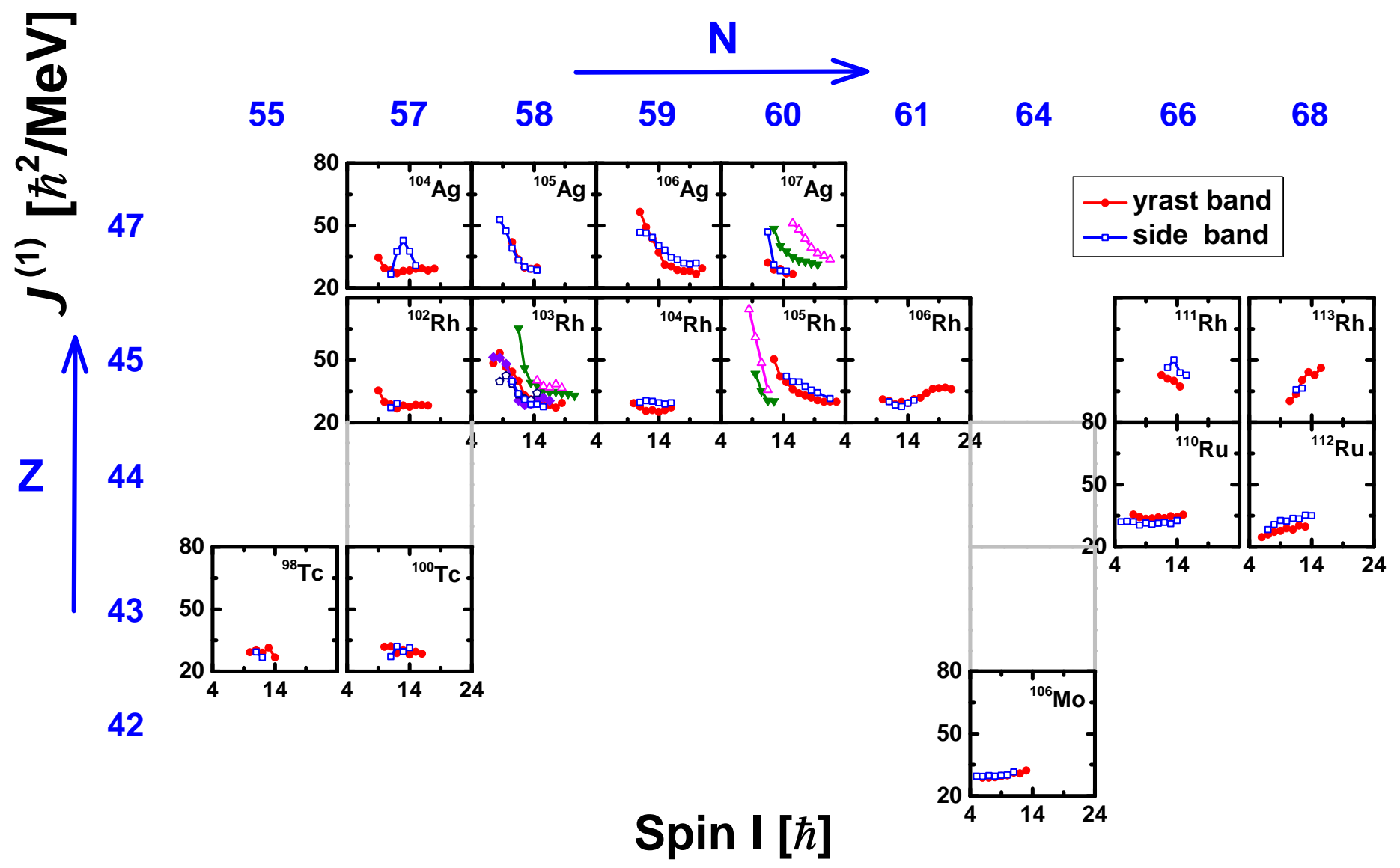

Fig. 20: (Color online) Kinematic moments of inertia versus spin for chiral doublet bands in $A \sim 100$ mass region. 


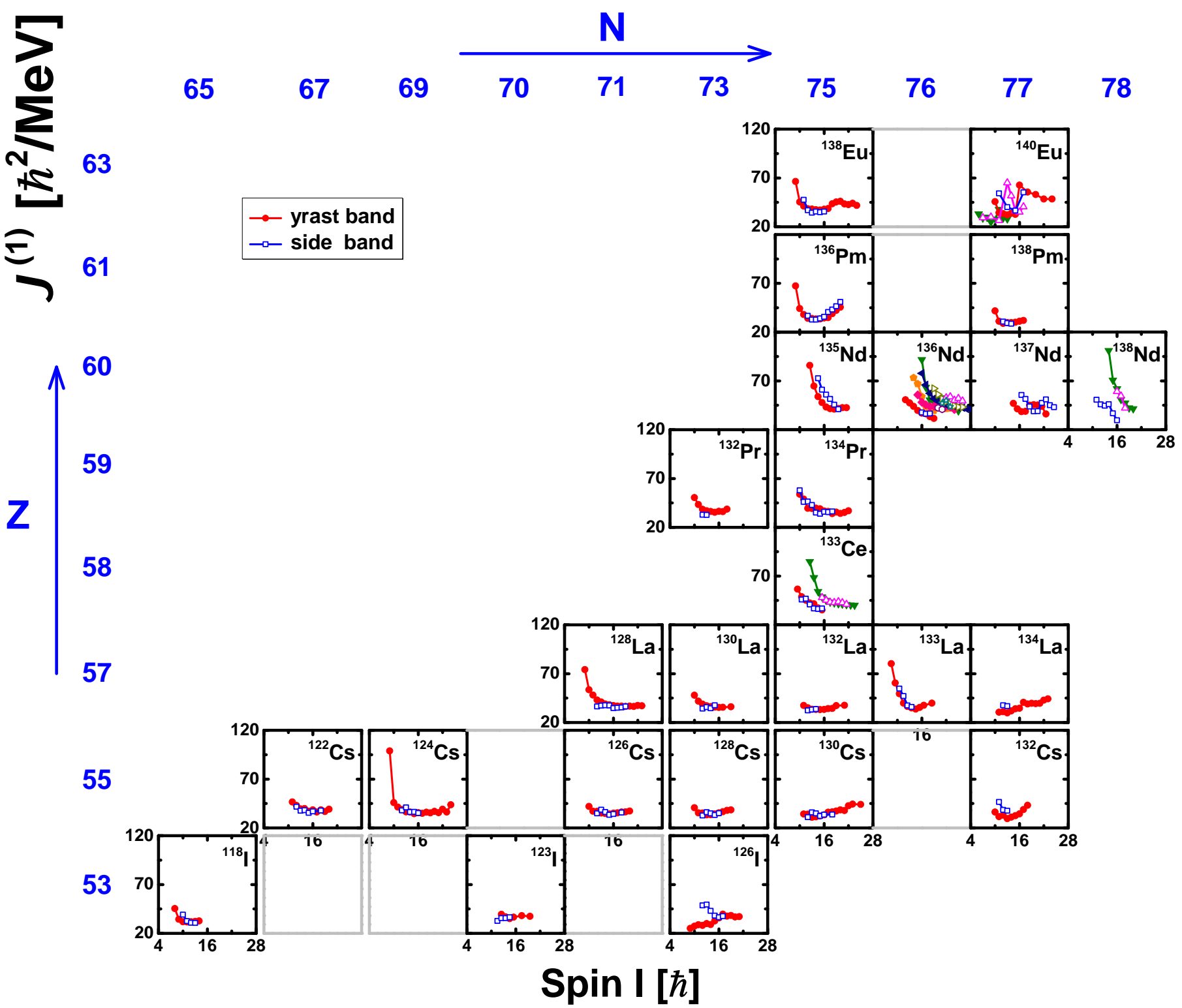

Fig. 21: (Color online) Kinematic moments of inertia versus spin for chiral doublet bands in $A \sim 130$ mass region. 


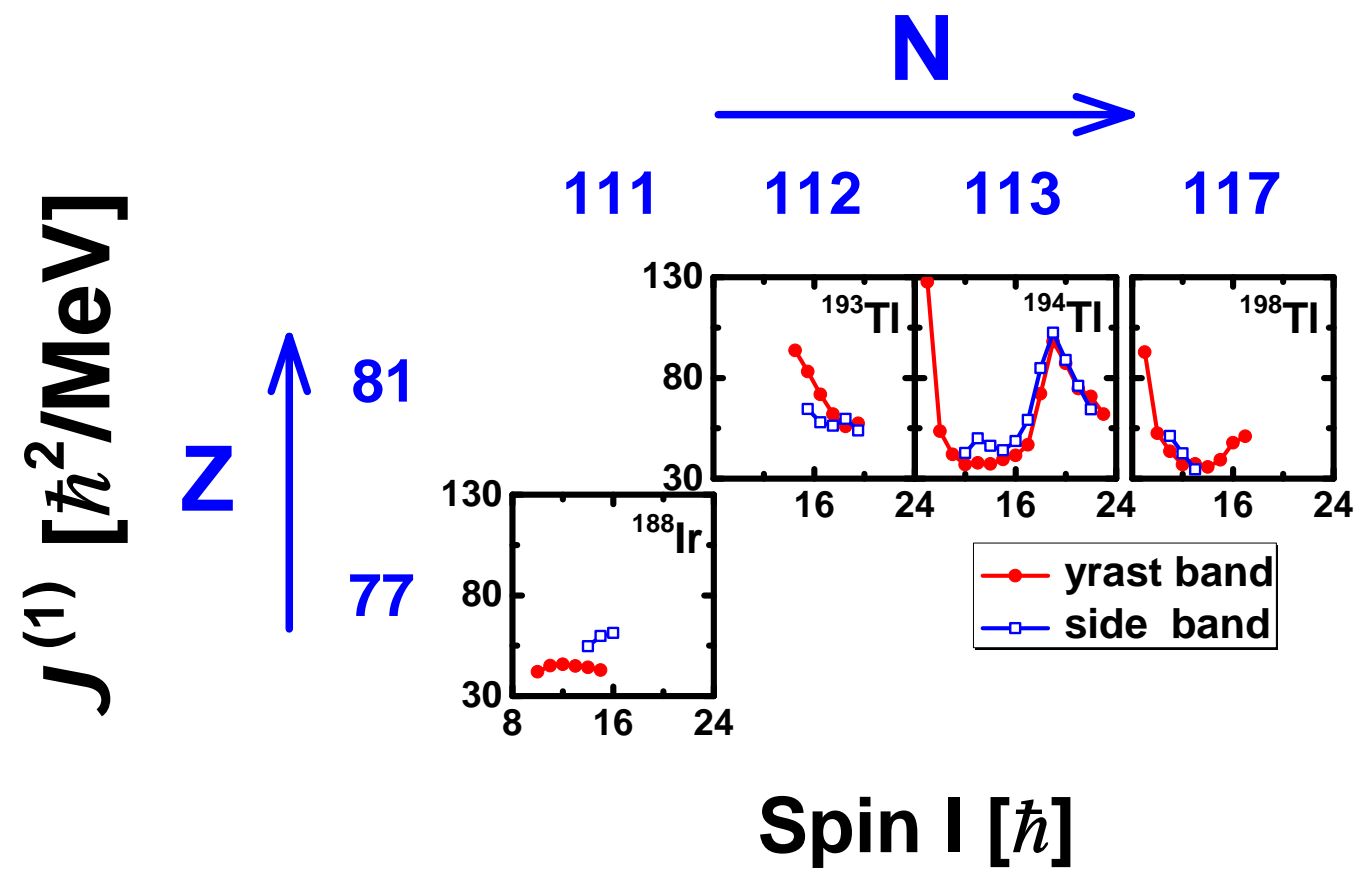

Fig. 22: (Color online) Kinematic moments of inertia versus spin for chiral doublet bands in $A \sim 190$ mass region. 


\subsection{Dynamic moment of inertia}

From the definition $\mathcal{J}^{(2)}(I)=\hbar \mathrm{d} I / \mathrm{d} \omega(I)$, the dynamic moments of inertia $\mathcal{J}^{(2)}$ for all chiral doublet bands in $A \sim 80,100,130$, and 190 mass regions are shown in Figs. 23-26, respectively.

Since the $\mathcal{J}^{(2)}$ corresponds to the second derivative of the energy with the spin, a large fluctuation exists. Nevertheless, similarities between the yrast bands and the side bands still exist in most chiral doublet bands.

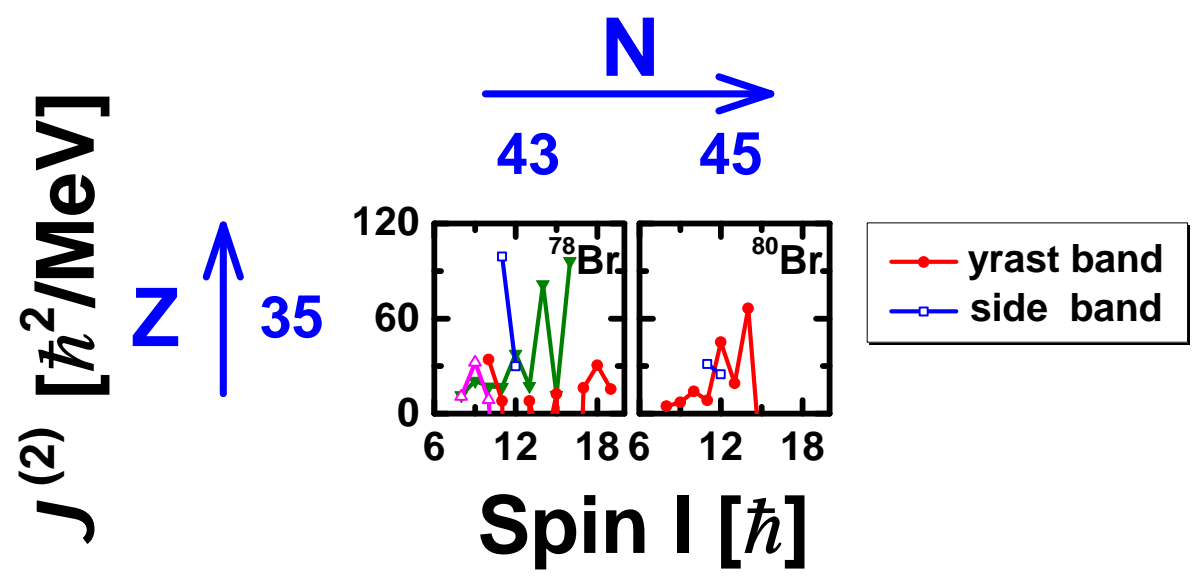

Fig. 23: (Color online) Dynamic moments of inertia versus spin for chiral doublet bands in $A \sim 80$ mass region. 


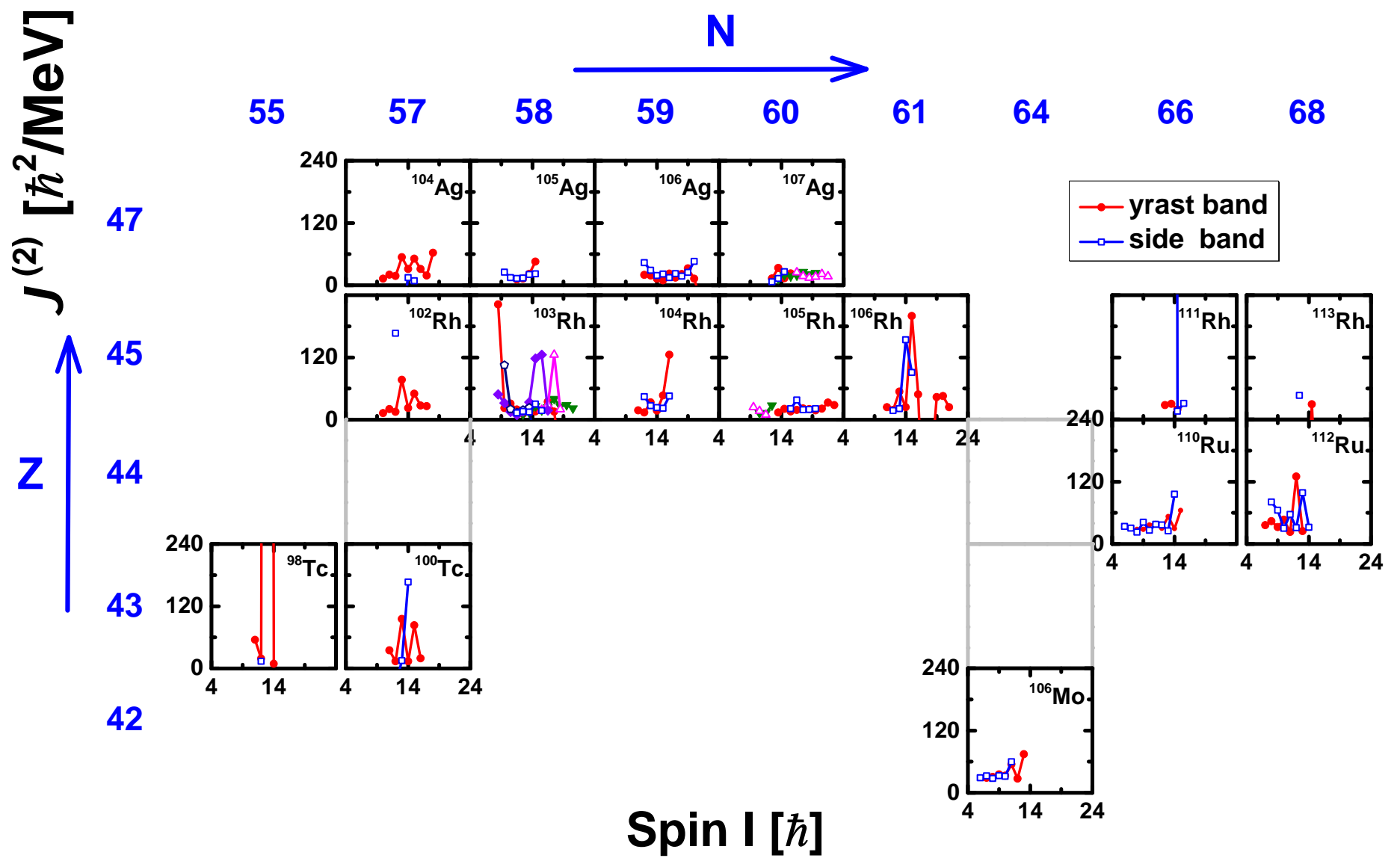

Fig. 24: (Color online) Dynamic moments of inertia versus spin for chiral doublet bands in $A \sim 100$ mass region. 


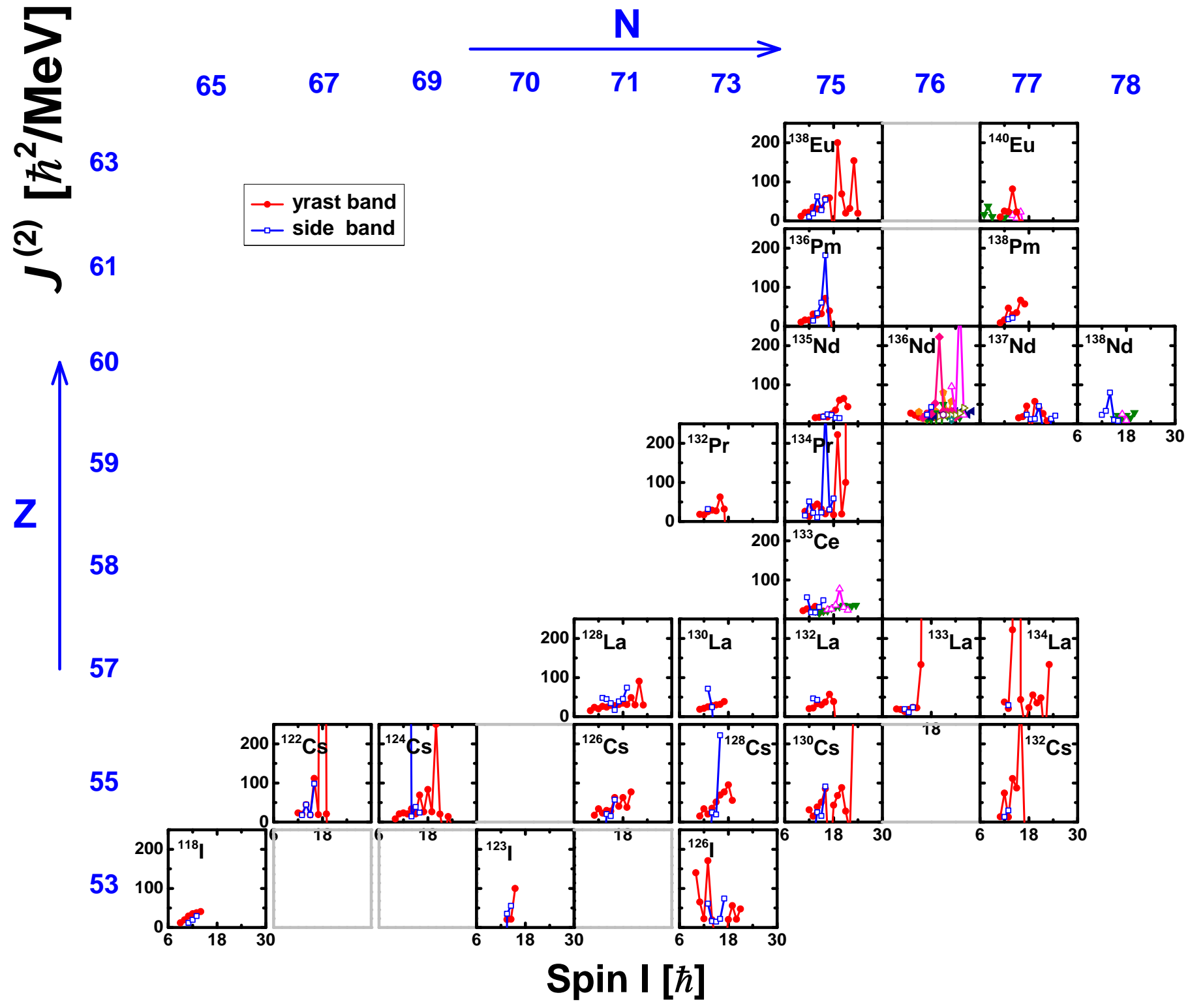

Fig. 25: (Color online) Dynamic moments of inertia versus spin for chiral doublet bands in $A \sim 130$ mass region. 


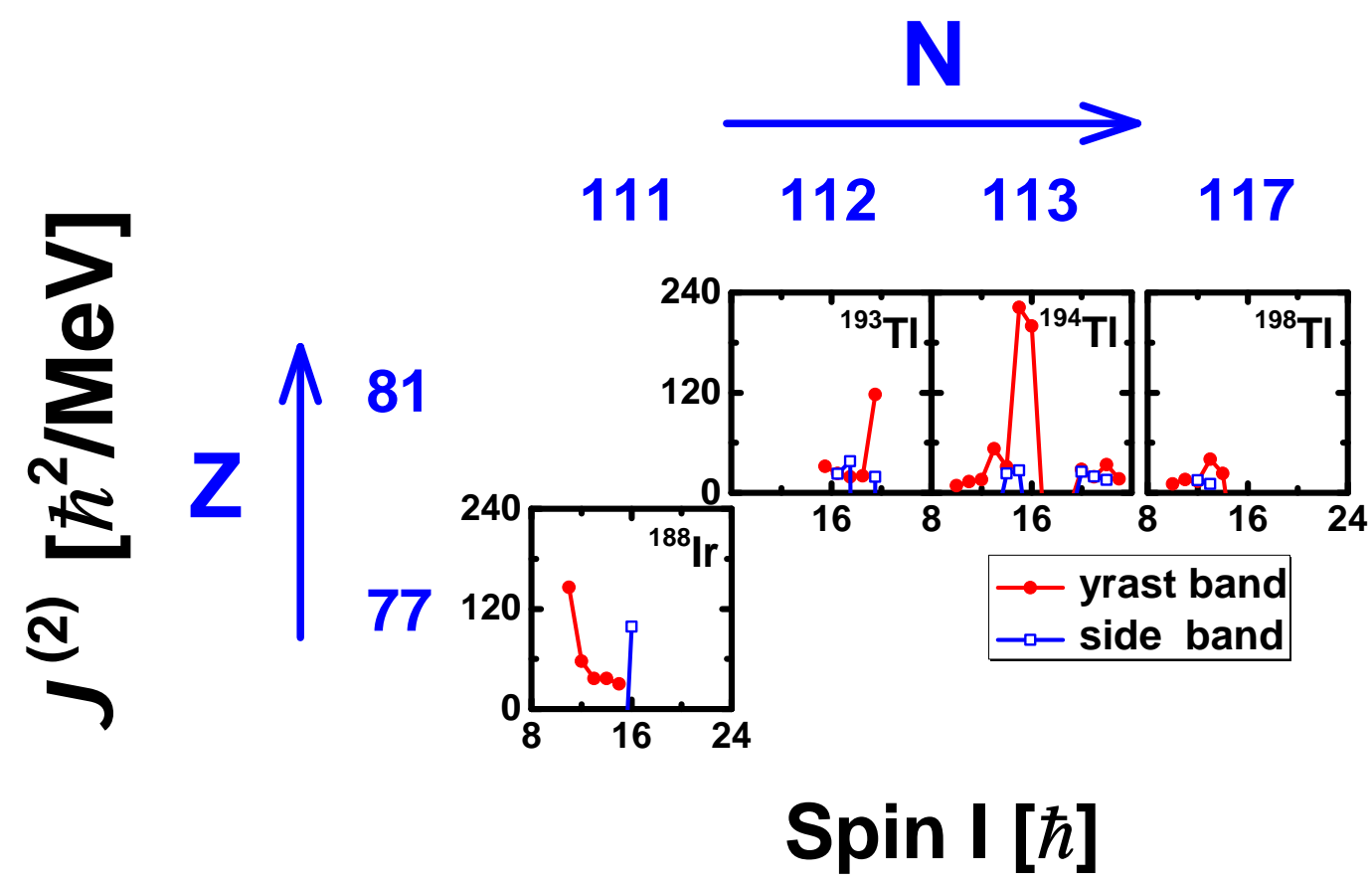

Fig. 26: (Color online) Dynamic moments of inertia versus spin for chiral doublet bands in $A \sim 190$ mass region. 


\subsection{Electromagnetic transition probability}

The ratios of the magnetic dipole transition strength to the electric quadrupole transition strength $B(M 1) / B(E 2)$ for all chiral doublet bands in $A \sim 80,100,130$, and 190 mass regions are given in Figs. 27-30, respectively. For the ideal chiral bands, due to the restoration of the chiral symmetry in the laboratory frame there are phase consequences for the chiral wavefunctions resulting in $M 1$ and $E 2$ selection rules which can manifest as $B(M 1) / B(E 2)$ ratios staggering as a function of spin. And the $B(M 1) / B(E 2)$ ratios are expected to be very similar for the chiral partner bands [73].

For the nuclei ${ }^{106} \mathrm{Mo},{ }^{98} \mathrm{Tc},{ }^{100} \mathrm{Tc},{ }^{110} \mathrm{Ru},{ }^{112} \mathrm{Ru},{ }^{102} \mathrm{Rh},{ }^{106} \mathrm{Rh},{ }^{111} \mathrm{Rh},{ }^{113} \mathrm{Rh},{ }^{104} \mathrm{Ag},{ }^{126} \mathrm{I},{ }^{128} \mathrm{Cs},{ }^{132} \mathrm{Cs},{ }^{132} \mathrm{La},{ }^{134} \mathrm{La}$, ${ }^{134} \mathrm{Pr},{ }^{136} \mathrm{Nd},{ }^{137} \mathrm{Nd},{ }^{138} \mathrm{Nd},{ }^{188} \mathrm{Ir}$, and ${ }^{198} \mathrm{Tl}$, the $B(M 1) / B(E 2)$ ratios are extracted by the equation (2) in Ref. [113]. For the nuclei ${ }^{104} \mathrm{Rh},{ }^{106} \mathrm{Ag},{ }^{107} \mathrm{Ag},{ }^{124} \mathrm{Cs},{ }^{126} \mathrm{Cs},{ }^{130} \mathrm{Cs},{ }^{135} \mathrm{Nd}$, and ${ }^{194} \mathrm{Tl}$ with $B(M 1)$ and $B(E 2)$ values available, the $B(M 1) / B(E 2)$ ratios are also calculated. The other data available are from the original references. The staggering of the $B(M 1) / B(E 2)$ ratios exists in most chiral doublet bands.

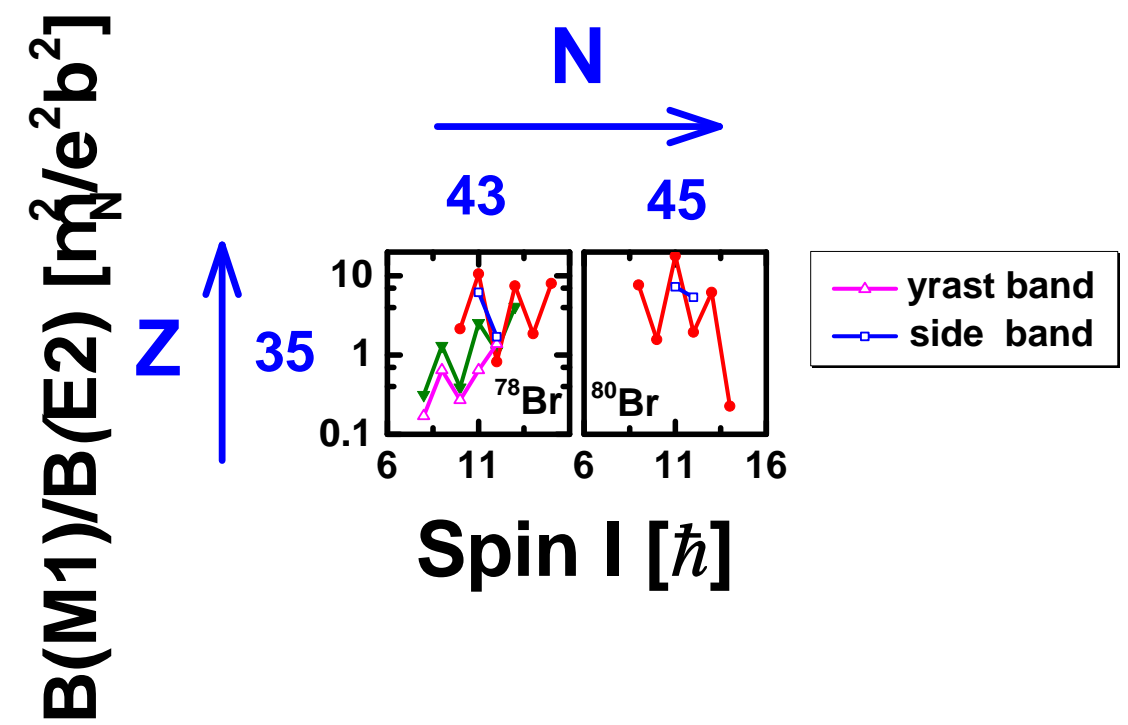

Fig. 27: (Color online) $B(M 1) / B(E 2)$ ratios versus spin for chiral doublet bands in the $A \sim 80$ mass region. 


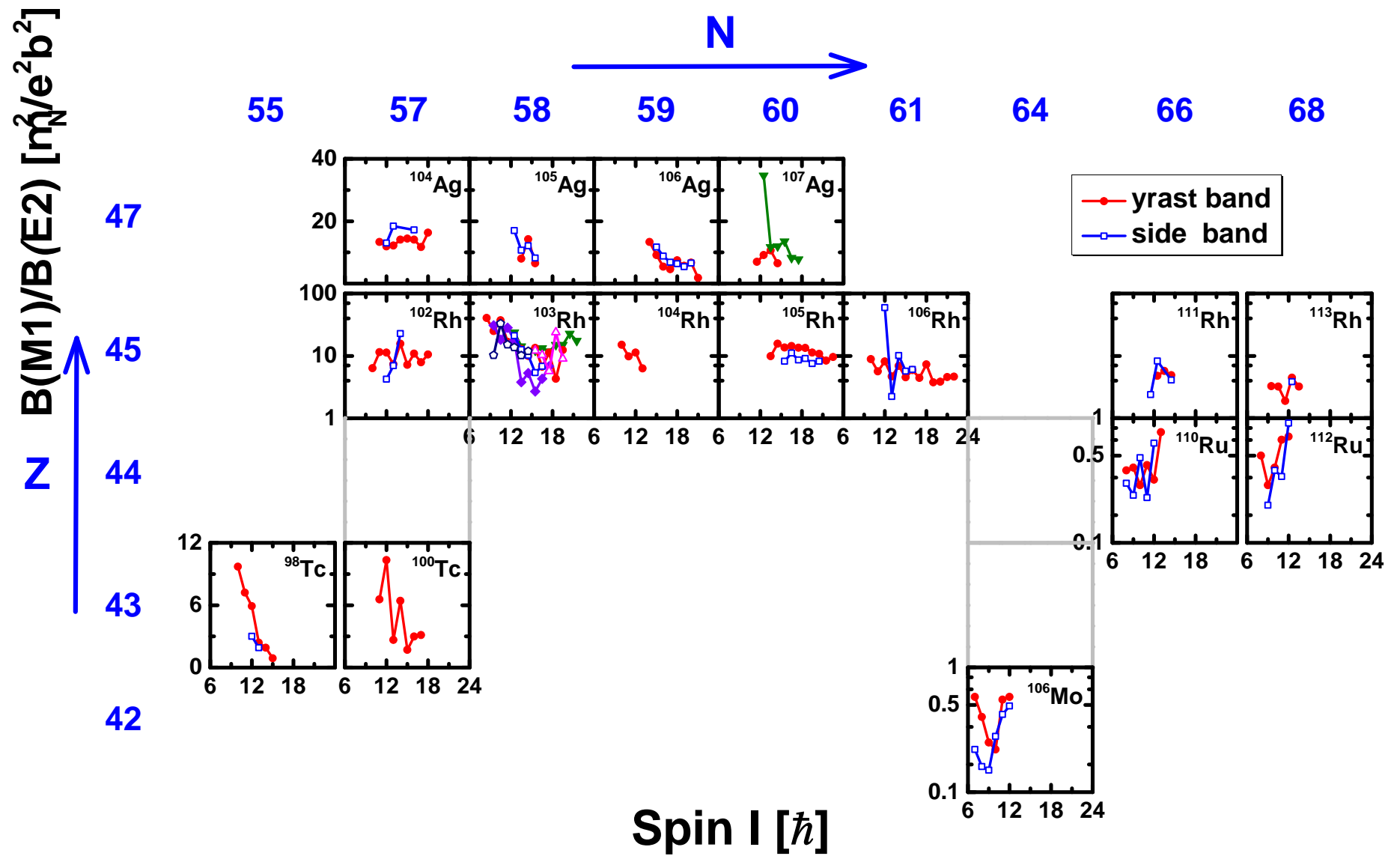

Fig. 28: (Color online) $B(M 1) / B(E 2)$ ratios versus spin for chiral doublet bands in the $A \sim 100$ mass region. 


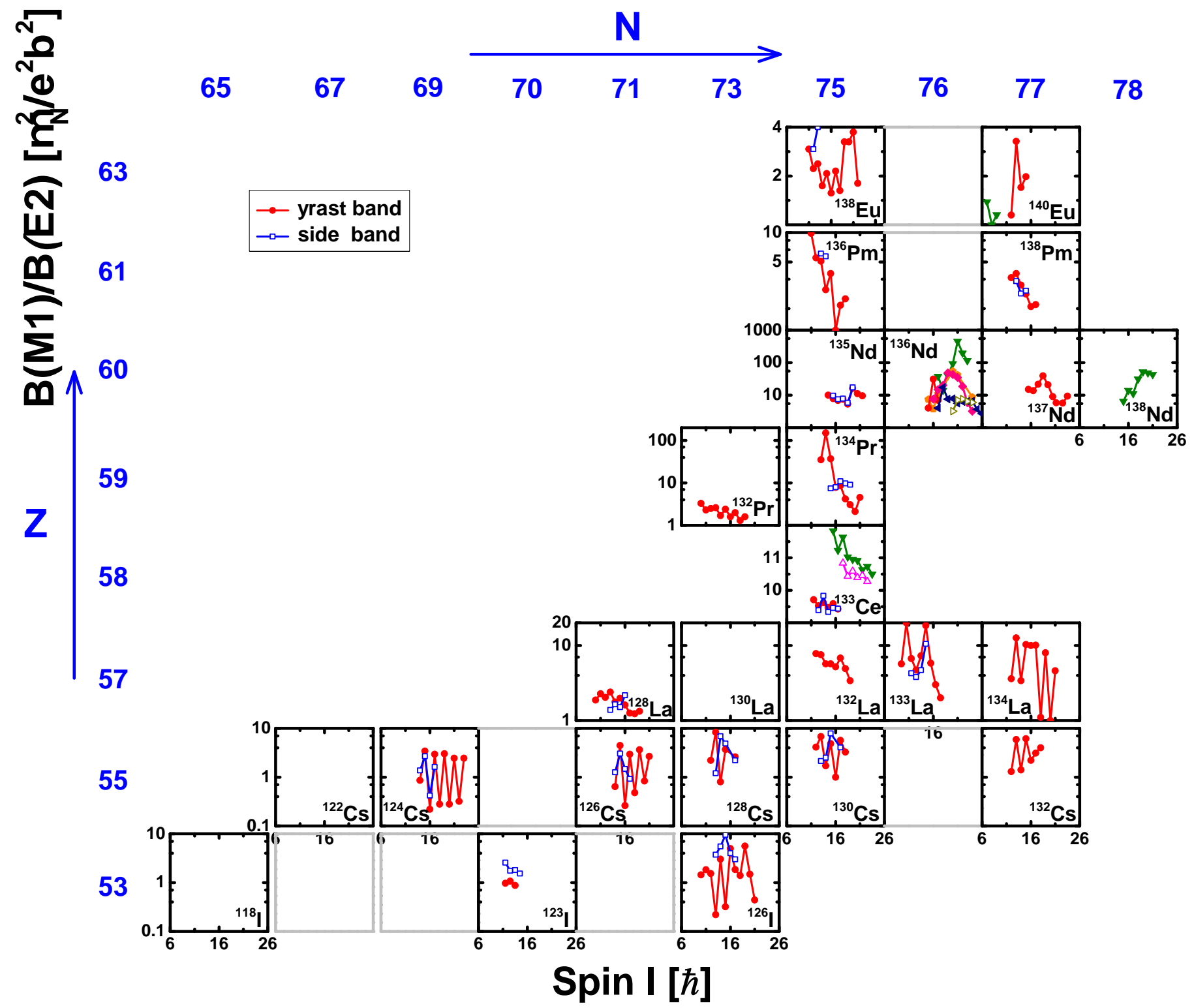

Fig. 29: (Color online) $B(M 1) / B(E 2)$ ratios versus spin for chiral doublet bands in the $A \sim 130$ mass region. 

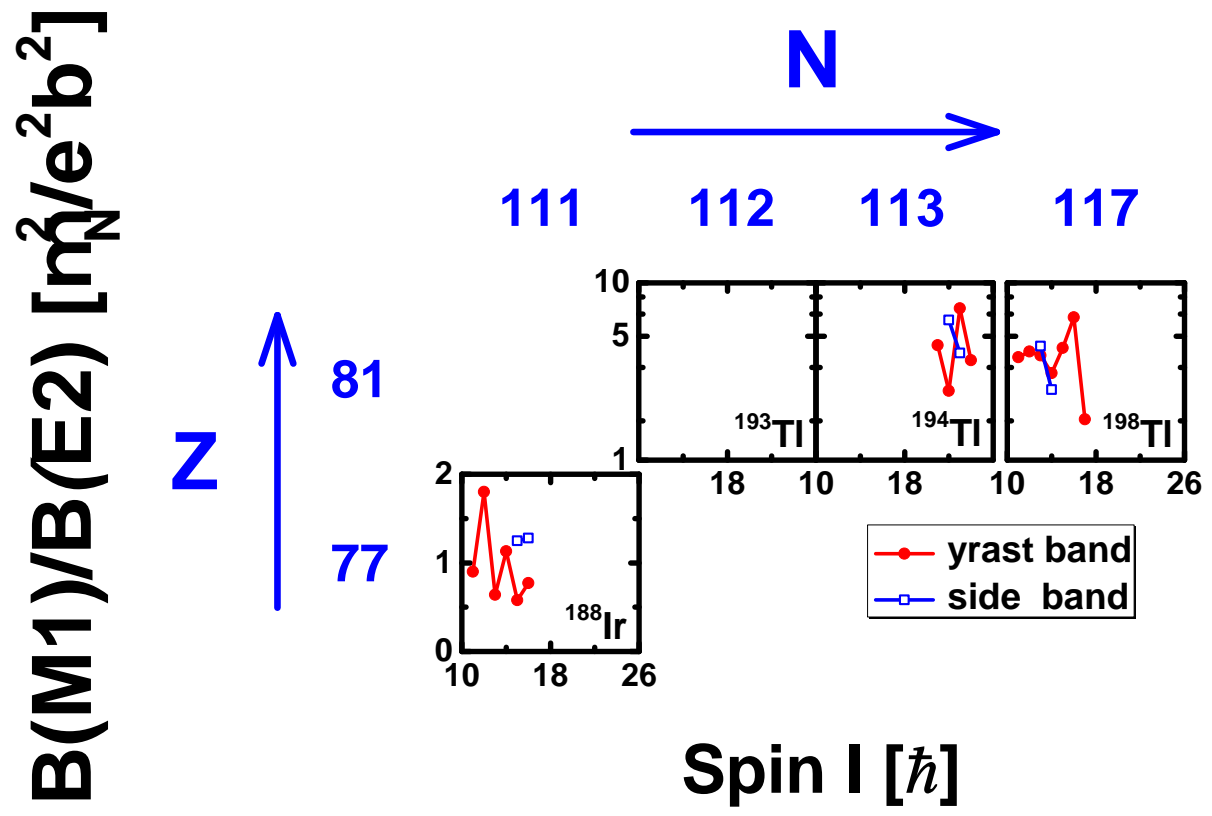

Fig. 30: (Color online) $B(M 1) / B(E 2)$ ratios versus spin for chiral doublet bands in the $A \sim 190$ mass region. 


\section{Summary}

Since the prediction of nuclear chirality in 1997, the nuclear chirality has become one of the hot topics in current nuclear physics frontiers. Experimentally, 59 chiral doublet bands in 47 chiral nuclei (including 8 nuclei with multiple chiral doublets) have been reported in $A \sim 80,100,130$, and 190 mass regions.

The spins, parities, energies, ratios of the magnetic dipole transition strengths to the electric quadrupole transition strengths, and related references for these nuclei have been compiled and listed in Table 1. For these nuclei with the magnetic dipole transition strengths and the electric quadrupole transition strengths measured, the corresponding results have been given in Table 2. A brief discussion has been provided after the presentation of energy $E$, energy difference $\Delta E$, energy staggering parameter $S(I)$, rotational frequency $\omega$, kinematic moment of inertia $\mathcal{J}^{(1)}$, dynamic moment of inertia $\mathcal{J}^{(2)}$, and ratio of the magnetic dipole transition strength to the electric quadrupole transition strength $B(M 1) / B(E 2)$ versus spin $I$ in each mass region.

\section{Acknowledgments}

The authors are indebted to Prof. J. Meng for the suggestion of this topic and the guidance during this work. The authors express thanks to R. Bark, U. Garg, A. A. Hecht, P. Joshi, T. Koike, C. Liu, M. L. Liu, J. B. Lu, Y. X. Luo, K. Y. Ma, Y. J. Ma, G. Rainovski, A. K. Singh, K. Starosta, J. Timár, B. Wadsworth, S. Y. Wang, Y. Zheng, L. H. Zhu, S. F. Zhu, and S. J. Zhu for providing the data and helpful suggestions. Fruitful discussions with F. Q. Chen, Q. B. Chen, Z. Shi, Y. K. Wang, X. H. Wu, S. Q. Zhang, Z. H. Zhang, and P. W. Zhao are very much appreciated. This work is supported in part by the Major State 973 Program of China (Grant No. 2013CB834400) and the National Natural Science Foundation of China (Grants No. 11335002, No. 11375015, No. 11461141002, and No. 11621131001).

\section{References}

\section{References}

[1] J. Meng (Ed.), Relativistic Density Functional for Nuclear Structure, International Review of Nuclear Physics, vol. 10, World Scientific, Singapore, 2016, pp. 387-411.

[2] S. Frauendorf, J. Meng, Nuclear Phys. A, 617 (1997) 131.

[3] J. Meng, S. Q. Zhang, J. Phys. G: Nucl. Part. Phys. 37 (2010) 064025.

[4] S. Frauendorf, Rev. Mod. Phys. 73 (2001) 463.

[5] J. Meng, Int. J. Mod. Phys. E, 20 (2011) 341.

[6] J. Meng, P. W. Zhao, Phys. Scr. 91 (2016) 053008.

[7] S. G. Zhou, Phys. Scr. 91 (2016) 063008.

[8] J. A. Sheikh, G. H. Bhat, W. A. Dar, S. Jehangir, P. A. Ganai, Phys. Scr. 91 (2016) 063015.

[9] A. A. Raduta, Prog. Part. Nucl. Phys. 90 (2016) 241.

[10] K. Starosta, T. Koike, Phys. Scr. 92 (2017) 093002. 
[11] J. Peng, J. Meng, S. Q. Zhang, Phys. Rev. C 68 (2003) 044324.

[12] T. Koike, K. Starosta, I. Hamamoto, Phys. Rev. Lett. 93 (2004) 172502.

[13] B. Qi, S. Q. Zhang, S. Y. Wang, J. M. Yao, J. Meng, Phys. Rev. C 79 (2009) 041302(R).

[14] B. Qi, S. Q. Zhang, S. Y. Wang, J. Meng, Chin. Phys. Lett. 27 (2010) 112101.

[15] Q. B. Chen, J. M. Yao, S. Q. Zhang, B. Qi, Phys. Rev. C 82 (2010) 067302.

[16] S. Q. Zhang, B. Qi, S. Y. Wang, J. Meng, Phys. Rev. C 75 (2007) 044307.

[17] S. Y. Wang, S. Q. Zhang, B. Qi, J. Peng, J. M. Yao, J. Meng, Phys. Rev. C 77 (2008) 034314.

[18] S. Y. Wang, B. Qi, D. P. Sun, Phys. Rev. C 82 (2010) 027303.

[19] B. Qi, S. Y. Wang, S. Q. Zhang, Chin. Phys. Lett. 28 (2011) 122101.

[20] B. Qi, S. Q. Zhang, J. Meng, S. Y. Wang, S. Frauendorf, Phys. Lett. B 675 (2009) 175.

[21] B. Qi, S. Q. Zhang, S. Y. Wang, J. Meng, T. Koike, Phys. Rev. C 83 (2011) 034303.

[22] B. Qi, J. Li, S. Y. Wang, J. Zhang, S. Q. Zhang, Chin. Phys. Lett. 29 (2012) 072101.

[23] K. Starosta, C. J. Chiara, D. B. Fossan, T. Koike, T. T. S. Kuo, D. R. LaFosse, S. G. Rohoziński, Ch. Droste, T. Morek, J. Srebrny, Phys. Rev. C 65 (2002) 044328.

[24] T. Koike, K. Starosta, C. J. Chiara, D. B. Fossan, D. R. LaFosse, Phys. Rev. C 67 (2003) 044319.

[25] Ch. Droste, S. G. Rohoziński, K. Starosta, L. Próchniak, E. Grodner, Eur. Phys. J. A 42 (2009) 79.

[26] D. Tonev, G. de Angelis, P. Petkov, A. Dewald, S. Brant, S. Frauendorf, D. L. Balabanski, P. Pejovic, D. Bazzacco, P. Bednarczyk, F. Camera, A. Fitzler, A. Gadea, S. Lenzi, S. Lunardi, N. Marginean, O. Möller, D. R. Napoli, A. Paleni, C. M. Petrache, G. Prete, K. O. Zell, Y. H. Zhang, Jing-ye Zhang, Q. Zhong, D. Curien, Phys. Rev. Lett. 96 (2006) 052501.

[27] D. Tonev, G. de Angelis, S. Brant, S. Frauendorf, P. Petkov, A. Dewald, F. Dönau, D. L. Balabanski, Q. Zhong, P. Pejovic, D. Bazzacco, P. Bednarczyk, F. Camera, D. Curien, F. Della Vedova, A. Fitzler, A. Gadea, G. Lo Bianco, S. Lenzi, S. Lunardi, N. Marginean, O. Möller, D. R. Napoli, R. Orlandi, E. Sahin, A. Saltarelli, J. Valiente Dobon, K. O. Zell, Jing-ye Zhang, Y. H. Zhang, Phys. Rev. C 76 (2007) 044313.

[28] S. Brant, D. Tonev, G. De Angelis, A. Ventura, Phys. Rev. C 78 (2008) 034301.

[29] S. Brant, C. M. Petrache, Phys. Rev. C 79 (2009) 054326.

[30] A. A. Raduta, Al. H. Raduta, C. M. Petrache, J. Phys. G: Nucl. Part. Phys. 43 (2016) 095107.

[31] M. Shimada, Y. Fujioka, S. Tagami, Y. R. Shimizu, Phys. Rev. C 97 (2018) 024319.

[32] K. Higashiyama, N. Yoshinaga, K. Tanabe, Phys. Rev. C 72 (2005) 024315.

[33] K. Higashiyama, N. Yoshinaga, Phys. Rev. C 88 (2013) 034315.

[34] V. I. Dimitrov, S. Frauendorf, F. Dönau, Phys. Rev. Lett. 84 (2000) 5732.

[35] H. Madokoro, J. Meng, M. Matsuzaki, S. Yamaji, Phys. Rev. C 62 (2000) 061301(R). 
[36] J. Meng, J. Peng, S. Q. Zhang, P. W. Zhao, Front. Phys. 8 (2013) 55.

[37] P. Olbratowski, J. Dobaczewski, J. Dudek, W. Płóciennik, Phys. Rev. Lett. 93 (2004) 052501.

[38] S. Mukhopadhyay, D. Almehed, U. Garg, S. Frauendorf, T. Li, P. V. Madhusudhana Rao, X. Wang, S. S. Ghugre, M. P. Carpenter, S. Gros, A. Hecht, R. V. F. Janssens, F. G. Kondev, T. Lauritsen, D. Seweryniak, S. Zhu, Phys. Rev. Lett. 99 (2007) 172501.

[39] D. Almehed, F. Dönau, S. Frauendorf, Phys. Rev. C 83 (2011) 054308.

[40] Q. B. Chen, S. Q. Zhang, P. W. Zhao, R. V. Jolos, J. Meng, Phys. Rev. C 87 (2013) 024314.

[41] Q. B. Chen, S. Q. Zhang, P. W. Zhao, R. V. Jolos, J. Meng, Phys. Rev. C 94 (2016) 044301.

[42] K. Hara, Y. Sun, Int. J. Mod. Phys. E 4 (1995) 637.

[43] G. H. Bhat, R. N. Ali, J. A. Sheikh, R. Palit, Nuclear Phys. A 922 (2014) 150.

[44] F. Q. Chen, Q. B. Chen, Y. A. Luo, J. Meng, S. Q. Zhang, Phys. Rev. C 96 (2017) 051303(R).

[45] F. Q. Chen, J. Meng, Acta Phys. Pol. B 11 (2018) 1001.

[46] P. Ring, Prog. Part. Nucl. Phys. 37 (1996) 193.

[47] J. Meng, H. Toki, S. G. Zhou, S. Q. Zhang, W. H. Long, L. S. Geng, Prog. Part. Nucl. Phys. 57 (2006) 470.

[48] J. Meng, S. G. Zhou, J. Phys. G: Nucl. Part. Phys. 42 (2015) 093101.

[49] H. Z. Liang, J. Meng, S. G. Zhou, Phys. Rep. 570 (2015) 1.

[50] J. Meng, J. Peng, S. Q. Zhang, S. G. Zhou, Phys. Rev. C 73 (2006) 037303.

[51] J. M. Yao, B. Qi, S. Q. Zhang, J. Peng, S. Y. Wang, J. Meng, Phys. Rev. C 79 (2009) 067302.

[52] J. Peng, H. Sagawa, S. Q. Zhang, J. M. Yao, Y. Zhang, J. Meng, Phys. Rev. C 77 (2008) 024309.

[53] A. D. Ayangeakaa, U. Garg, M. D. Anthony, S. Frauendorf, J. T. Matta, B. K. Nayak, D. Patel, Q. B. Chen, S. Q. Zhang, P. W. Zhao, B. Qi, J. Meng, R. V. F. Janssens, M. P. Carpenter, C. J. Chiara, F. G. Kondev, T. Lauritsen, D. Seweryniak, S. Zhu, S. S. Ghugre, R. Palit, Phys. Rev. Lett. 110 (2013) 172504.

[54] I. Kuti, Q. B. Chen, J. Timár, D. Sohler, S. Q. Zhang, Z. H. Zhang, P. W. Zhao, J. Meng, K. Starosta, T. Koike, E. S. Paul, D. B. Fossan, C. Vaman, Phys. Rev. Lett. 113 (2014) 032501.

[55] C. Liu, S. Y. Wang, R. A. Bark, S. Q. Zhang, J. Meng, B. Qi, P. Jones, S. M. Wyngaardt, J. Zhao, C. Xu, S.-G. Zhou, S. Wang, D. P. Sun, L. Liu, Z. Q. Li, N. B. Zhang, H. Jia, X. Q. Li, H. Hua, Q. B. Chen, Z. G. Xiao, H. J. Li, L. H. Zhu, T. D. Bucher, T. Dinoko, J. Easton, K. Juhász, A. Kamblawe, E. Khaleel, N. Khumalo, E. A. Lawrie, J. J. Lawrie, S. N. T. Majola, S. M. Mullins, S. Murray, J. Ndayishimye, D. Negi, S. P. Noncolela, S. S. Ntshangase, B. M. Nyakó, J. N. Orce, P. Papka, J. F. Sharpey-Schafer, O. Shirinda, P. Sithole, M. A. Stankiewicz, M. Wiedeking, Phys. Rev. Lett. 116 (2016) 112501.

[56] C. M. Petrache, B. F. Lv, A. Astier, E. Dupont, Y. K. Wang, S. Q. Zhang, P. W. Zhao, Z. X. Ren, J. Meng, P. T. Greenlees, H. Badran, D. M. Cox, T. Grahn, R. Julin, S. Juutinen, J. Konki, J. Pakarinen, P. Papadakis, J. Partanen, P. Rahkila, M. Sandzelius, J. Saren, C. Scholey, J. Sorri, S. Stolze, J. Uusitalo, B. Cederwall, Ö. 
Aktas, A. Ertoprak, H. Liu, S. Matta, P. Subramaniam, S. Guo, M. L. Liu, X. H. Zhou, K. L. Wang, I. Kuti, J. Timár, A. Tucholski, J. Srebrny, C. Andreoiu, Phys. Rev. C 97 (2018) 041304(R).

[57] J. A. Alcántara-Núñez, J. R. B. Oliveira, E. W. Cybulska, N. H. Medina, M. N. Rao, R. V. Ribas, M. A. Rizzutto, W. A. Seale, F. Falla-Sotelo, K. T. Wiedemann, V. I. Dimitrov, S. Frauendorf, Phys. Rev. C 69 (2004) 024317.

[58] J. Timár, P. Joshi, K. Starosta, V. I. Dimitrov, D. B. Fossan, J. Molnár, D. Sohler, R. Wadsworth, A. Algora, P. Bednarczyk, D. Curieng, Zs. Dombrádi, G. Duchene, A. Gizon, J. Gizon, D. G. Jenkins, T. Koike, A. Krasznahorkay, E. S. Paul, P. M. Raddon, G. Rainovski, J. N. Scheurer, A. J. Simons, C. Vaman, A. R. Wilkinson, L. Zolnai, S. Frauendorf, Phys. Lett. B 598 (2004) 178.

[59] J. Li, S. Q. Zhang, J. Meng, Phys. Rev. C 83 (2011) 037301.

[60] C. Y. He, B. Zhang, L. H. Zhu, X. G. Wu, H. B. Sun, Y. Zheng, B. B. Yu, L. L. Wang, G. S. Li, S. H. Yao, C. Xu, J. G. Wang, L. Gu, Plasma Sci. Technol. 14 (2012) 518.

[61] Dan Jerrestam, W. Klamra, J. Gizon, F. Lidén, L. Hildingsson, J. Kownacki, Th. Lindblad, J. Nyberg, Nuclear Phys. A 577 (1994) 786.

[62] B. Qi, H. Jia, N. B. Zhang, C. Liu, S. Y. Wang, Phys. Rev. C 88 (2013) 027302.

[63] C. M. Petrache, S. Frauendorf, M. Matsuzaki, R. Leguillon, T. Zerrouki, S. Lunardi, D. Bazzacco, C. A. Ur, E. Farnea, C. Rossi Alvarez, R. Venturelli, G. de Angelis, Phys. Rev. C 86 (2012) 044321.

[64] J. Peng, J. Meng, P. Ring, S. Q. Zhang, Phys. Rev. C 78 (2008) 024313.

[65] P. W. Zhao, S. Q. Zhang, J. Peng, H. Z. Liang, P. Ring, J. Meng, Phys. Lett. B 699 (2011) 181.

[66] L. F. Yu, P. W. Zhao, S. Q. Zhang, P. Ring, J. Meng, Phys. Rev. C 85 (2012) 024318.

[67] P. W. Zhao, J. Peng, H. Z. Liang, P. Ring, J. Meng, Phys. Rev. Lett. 107 (2011) 122501.

[68] P. W. Zhao, J. Peng, H. Z. Liang, P. Ring, J. Meng, Phys. Rev. C 85 (2012) 054310.

[69] P. W. Zhao, S. Q. Zhang, J. Meng, Phys. Rev. C 92 (2015) 034319.

[70] Y. K. Wang, Phys. Rev. C 96 (2017) 054324.

[71] P. W. Zhao, N. Itagaki, J. Meng, Phys. Rev. Lett. 115 (2015) 022501.

[72] P. W. Zhao, Phys. Lett. B 773 (2017) 1.

[73] S. Y. Wang, S. Q. Zhang, B. Qi, J. Meng, Chin. Phys. Lett. 24 (2007) 664.

[74] S. Y. Wang, B. Qi, L. Liu, S. Q. Zhang, H. Hua, X. Q. Li, Y. Y. Chen, L. H. Zhu, J. Meng, S. M. Wyngaardt, P. Papka, T. T. Ibrahim, R. A. Bark, P. Datta, E. A. Lawrie, J. J. Lawrie, S. N. T. Majola, P. L. Masiteng, S. M. Mullins, J. Gál, G. Kalinka, J. Molnár, B. M. Nyakó, J. Timár, K. Juhász, R. Schwengner, Phys. Lett. B 703 (2011) 40 .

[75] S. J. Zhu, J. H. Hamilton, A. V. Ramayya, J. K. Hwang, J. O. Rasmussen, Y. X. Luo, K. Li, J. G. Wang, X. L. Che, H. B. Ding, S. Frauendorf, V. Dimitrov, Q. Xu, L. Gu, Y. Y. Yang, Chin. Phys. C 33 (2009) 145.

[76] H. B. Ding, S. J. Zhu, J. G. Wang, L. Gu, Q. Xu, Z. G. Xiao, E. Y. Yeoha, M. Zhang, L. H. Zhu, X. G. Wu, 
Y. Liu, C. Y. He, L. L. Wang, B. Pan, G. S. Li, Chin. Phys. Lett. 27 (2010) 072501.

[77] P. Joshi, A. R. Wilkinson, T. Koike, D. B. Fossan, S. Finnigan, E. S. Paul, P. M. Raddon, G. Rainovski, K. Starosta, A. J. Simons, C. Vaman, R. Wadsworth, Eur. Phys. J. A 24 (2005) 23.

[78] Y. X. Luo, S. J. Zhu, J. H. Hamilton, A. V. Ramayya, C. Goodin, K. Li, X. L. Che, J. K. Hwang, I. Y. Lee, Z. Jiang, G. M. Ter-akopian, A. V. Daniel, M. A. Stoyer, R. Donangelo, S. Frauendorf, V. Dimitrov, Jing-ye Zhang, J. D. Cole, N. J. Stone, J. O. Rasmussen, Int. J. Mod. Phys. E 18 (2009) 1697.

[79] D. Tonev, M. S. Yavahchova, N. Goutev, G. de Angelis, P. Petkov, R. K. Bhowmik, R. P. Singh, S. Muralithar, N. Madhavan, R. Kumar, M. Kumar Raju, J. Kaur, G. Mohanto, A. Singh, N. Kaur, R. Garg, A. Shukla, Ts. K. Marinov, S. Brant. Phys. Rev. Lett. 112 (2014) 052501.

[80] C. Vaman, D. B. Fossan, T. Koike, K. Starosta, I. Y. Lee, A. O. Macchiavelli, Phys. Rev. Lett. 92 (2004) 032501.

[81] P. Joshi, D. G. Jenkins, P. M. Raddon, A. J. Simons, R. Wadsworth, A. R. Wilkinson, D. B. Fossan, T. Koike, K. Starosta, C. Vaman, J. Timár, Zs. Dombrádi, A. Krasznahorkay, J. Molnár, D. Sohler, L. Zolnai, A. Algora, E. S. Paul, G. Rainovski, A. Gizon, J. Gizon, P. Bednarczyk, D. Curien, G. Duchêne, J. N. Scheurer, Phys. Lett. B 595 (2004) 135.

[82] Y. X. Luo, S. C. Wu, J. Gilat, J. O. Rasmussen, J. H. Hamilton, A. V. Ramayya, J. K. Hwang, C. J. Beyer, S. J. Zhu, J. Kormicki, X. Q. Zhang, E. F. Jones, P. M. Gore, I-Yang Lee, P. Zielinski, C. M. Folden, T. N. Ginter, P. Fallon, G. M. Ter-Akopian, A. V. Daniel, M. A. Stoyer, J. D. Cole, R. Donangelo, S. J. Asztalos, A. Gelberg, Phys. Rev. C 69 (2004) 024315.

[83] Z. G. Wang, M. L. Liu, Y. H. Zhang, X. H. Zhou, B. T. Hu, N. T. Zhang, S. Guo, B. Ding, Y. D. Fang, J. G. Wang, G. S. Li, Y. H. Qiang, S. C. Li, B. S. Gao, Y. Zheng, W. Hua, X. G. Wu, C. Y. He, Y. Zheng, C. B. Li, J. J. Liu, S. P. Hu. Phys. Rev. C 88 (2013) 024306.

[84] J. Timár, T. Koike, N. Pietralla, G. Rainovski, D. Sohler, T. Ahn, G. Berek, A. Costin, K. Dusling, T. C. Li, E. S. Paul, K. Starosta, C. Vaman, Phys. Rev. C 76 (2007) 024307.

[85] E. O. Lieder, R. M. Lieder, R. A. Bark, Q. B. Chen, S. Q. Zhang, J. Meng, E. A. Lawrie, J. J. Lawrie, S. P. Bvumbi, N. Y. Kheswa, S. S. Ntshangase, T. E. Madiba, P. L. Masiteng, S. M. Mullins, S. Murray, P. Papka, D. G. Roux, O. Shirinda, Z. H. Zhang, P. W. Zhao, Z. P. Li, J. Peng, B. Qi, S. Y. Wang, Z. G. Xiao, C. Xu, Phys. Rev. Lett. 112 (2014) 202502.

[86] K. Starosta, T. Koike, C. J. Chiara, D. B. Fossan, D. R. LaFosse, Nuclear Phys. A 682 (2001) 375c.

[87] Y. X. Zhao, T. Komatsubara, Y. J. Ma, Y. H. Zhang, S .Y. Wang, Y. Z. Liu, K. Furuno, Chin. Phys. Lett. 26 (2009) 082301.

[88] Y. Zheng, L. H. Zhu, X. G. Wu, Z. C. Gao, C. Y. He, G. S. Li, L. L. Wang, Y. S. Chen, Y. Sun, X. Hao, Y. Liu, X. Q. Li, B. Pan, Y. J. Ma, Z. Y. Li, H. B. Ding, Phys. Rev. C 86 (2012) 014320.

[89] U. Yon-Nam, S. J. Zhu, M. Sakhaee, L. M. Yang, C. Y. Gan, L. Y. Zhu, R. Q. Xu, X. L. Che, M. L. Li, Y. J. Chen, S. X. Wen, X. G. Wu, L. H. Zhu, G. S. Li, J. Peng, S. Q. Zhang, J. Meng, J. Phys. G: Nucl. Part. Phys. 31 (2005) B1. 
[90] K. Selvakumar, A. K. Singh, Chandan Ghosh, Purnima Singh, A. Goswami, R. Raut, A. Mukherjee, U. Datta, P. Datta, S. Roy, G. Gangopadhyay, S. Bhowal, S. Muralithar, R. Kumar, R. P. Singh, M. Kumar Raju, Phys. Rev. C 92 (2015) 064307.

[91] E. Grodner, I. Sankowska, T. Morek, S. G. Rohoziński, Ch. Droste, J. Srebrny, A. A. Pasternak, M. Kisieliński, M. Kowalczyk, J. Kownacki, J. Mierzejewski, A. Król, K. Wrzosek, Phys. Lett. B 703 (2011) 46.

[92] E. Grodner, J. Srebrny, A. A. Pasternak, I. Zalewska, T. Morek, Ch. Droste, J. Mierzejewski, M. Kowalczyk, J. Kownacki, M. Kisieliński, S. G. Rohoziński, T. Koike, K. Starosta, A. Kordyasz, P. J. Napiorkowski, M. WolińskaCichocka, E. Ruchowska, W. Płóciennik, J. Perkowski, Phys. Rev. Lett. 97 (2006) 172501.

[93] A. J. Simons, P. Joshi, D. G. Jenkins, P. M. Raddon, R. Wadsworth, D. B. Fossan, T. Koike, C. Vaman, K. Starosta, E. S. Paul, H. J. Chantler, A. O. Evans, P. Bednarczyk, D. Curien, J. Phys. G: Nucl. Part. Phys. 31 (2005) 541.

[94] G. Rainovski, E. S. Paul, H. J. Chantler, P. J. Nolan, D. G. Jenkins, R. Wadsworth, P. Raddon, A. Simons, D. B. Fossan, T. Koike, K. Starosta, C. Vaman, E. Farnea, A. Gadea, Th. Kröll, R. Isocrate, G. de Angelis, D. Curien, V. I. Dimitrov, Phys. Rev. C 68 (2003) 024318.

[95] K. Y. Ma, J. B. Lu, D. Yang, H. D. Wang, Y. Z. Liu, X. G. Wu, Y. Zheng, C. Y. He, Phys. Rev. C 85 (2012) 037301.

[96] T. Koike, K. Starosta, C. J. Chiara, D. B. Fossan, D. R. LaFosse, Phys. Rev. C 63 (2001) 061304(R).

[97] I. Kuti, J. Timár, D. Sohler, E. S. Paul, K. Starosta, A. Astier, D. Bazzacco, P. Bednarczyk, A. J. Boston, N. Buforn, H. J. Chantler, C. J. Chiara, R. M. Clark, M. Cromaz, M. Descovich, Zs. Dombrádi, P. Fallon, D. B. Fossan, C. Fox, A. Gizon, J. Gizon, A. A. Hecht, N. Kintz, T. Koike, I. Y. Lee, S. Lunardi, A. O. Macchiavelli, P. J. Nolan, B. M. Nyakó, C. M. Petrache, J. A. Sampson, H. C. Scraggs, T. G. Tornyi, R. Wadsworth, A. Walker, L. Zolnai, Phys. Rev. C 87 (2013) 044323.

[98] C. M. Petrache, Q. B. Chen, S. Guo, A. D. Ayangeakaa, U. Garg, J. T. Matta, B. K. Nayak, D. Patel, J. Meng, M. P. Carpenter, C. J. Chiara, R. V. F. Janssens, F. G. Kondev, T. Lauritsen, D. Seweryniak, S. Zhu, S. S. Ghugre, R. Palit, Phys. Rev. C 94 (2016) 064309.

[99] R. A. Bark, A. M. Baxter, A. P. Byrne, G. D. Dracoulis, T. Kibédi, T. R. McGoram, S. M. Mullins, Nuclear Phys. A 691 (2001) 577 .

[100] J. Timár, K. Starosta, I. Kuti, D. Sohler, D. B. Fossan, T. Koike, E. S. Paul, A. J. Boston, H. J. Chantler, M. Descovich, R. M. Clark, M. Cromaz, P. Fallon, I. Y. Lee, A. O. Macchiavelli, C. J. Chiara, R. Wadsworth, A. A. Hecht, D. Almehed, S. Frauendorf, Phys. Rev. C 84 (2011) 044302.

[101] C. M. Petrache, R. Venturelli, D. Vretenar, D. Bazzacco, G. Bonsignori, S. Brant, S. Lunardi, M. A. Rizzutto, C. Rossi Alvarez, G. de Angelis, M. De Poli, D. R. Napoli, Nuclear Phys. A 617 (1997) 228.

[102] D. J. Hartley, L. L. Riedinger, M. A. Riley, D. L. Balabanski, F. G. Kondev, R. W. Laird, J. Pfohl, D. E. Archer, T. B. Brown, R. M. Clark, M. Devlin, P. Fallon, I. M. Hibbert, D. T. Joss, D. R. LaFosse, P. J. Nolan, N. J. O’Brien, E. S. Paul, D. G. Sarantites, R. K. Sheline, S. L. Shepherd, J. Simpson, R. Wadsworth, Jing-ye Zhang, P. B. Semmes, F. Dönau, Phys. Rev. C 64 (2001) 031304(R). 
[103] K. Y. Ma, J. B. Lu, Z. Zhang, J. Q. Liu, D. Yang, Y. M. Liu, X. Xu, X. Y. Li, Y. Z. Liu, X. G. Wu, Y. Zheng, C. B. Li, Phys. Rev. C 97 (2018) 014305.

[104] A. A. Hecht, C. W. Beausang, K. E. Zyromski, D. L. Balabanski, C. J. Barton, M. A. Caprio, R. F. Casten, J. R. Cooper, D. J. Hartley, R. Krücken, D. Meyer, H. Newman, J. R. Novak, E. S. Paul, N. Pietralla, A. Wolf, N. V. Zamfir, Jing-ye Zhang, F. Dönau, Phys. Rev. C 63 (2001) 051302(R).

[105] A. A. Hecht, C. W. Beausang, H. Amro, C. J. Barton, Z. Berant, M. A. Caprio, R. F. Casten, J. R. Cooper, D. J. Hartley, R. Krücken, D. A. Meyer, H. Newman, J. R. Novak, N. Pietralla, J. J. Ressler, A. Wolf, N. V. Zamfir, Jing-ye Zhang, K. E. Zyromski, Phys. Rev. C 68 (2003) 054310.

[106] D. L. Balabanski, M. Danchev, D. J. Hartley, L. L. Riedinger, O. Zeidan, Jing-ye Zhang, C. J. Barton, C. W. Beausang, M. A. Caprio, R. F. Casten, J. R. Cooper, A. A. Hecht, R. Krücken, J. R. Novak, N. V. Zamfir, K. E. Zyromski, Phys. Rev. C 70 (2004) 044305.

[107] J. Ndayishimye, E. A. Lawrie, O. Shirinda, J. L. Easton, S. M. Wyngaardt, R. A. Bark, S. P. Bvumbi, T. R. S. Dinoko, P. Jones, N. Y. Kheswa, J. J. Lawrie, S. N. T. Majola, P. L. Masiteng, D. Negi, J. N. Orce, P. Papka, J. F. Sharpey-Schafer, M. Stankiewicz, M. Wiedeking, Acta Phys. Pol. B 48 (2017) 343.

[108] P. L. Masiteng, E. A. Lawrie, T. M. Ramashidzha, J. J. Lawrie, R. A. Bark, R. Lindsay, F. Komati, J. Kau, P. Maine, S. M. Maliage, I. Matamba, S. M. Mullins, S. H. T. Murray, K. P. Mutshena, A. A. Pasternak, D. G. Roux, J. F. Sharpey-Schafer, O. Shirinda, P. A. Vymers, Eur. Phys. J. A 50 (2014) 119.

[109] E. A. Lawrie, P. A. Vymers, Ch. Vieu, J. J. Lawrie, C. Schück, R. A. Bark, R. Lindsay, G. K. Mabala, S. M. Maliage, P. L. Masiteng, S. M. Mullins, S. H. T. Murray, I. Ragnarsson, T. M. Ramashidzha, J. F. Sharpey-Schafer, O. Shirinda, Eur. Phys. J. A 45 (2010) 39.

[110] S. J. Zhu, J. H. Hamilton, A. V. Ramayya, P. M. Gore, J. O. Rasmussen, V. Dimitrov, S. Frauendorf, R. Q. Xu, J. K. Hwang, D. Fong, L. M. Yang, K. Li, Y. J. Chen, X. Q. Zhang, E. F. Jones, Y. X. Luo, I. Y. Lee, W. C. Ma, J. D. Cole, M. W. Drigert, M. Stoyer, G. M. Ter-Akopian, A. V. Daniel, Eur. Phys. J. A 25 (Suppl. 1) (2005) 459.

[111] Y. X. Luo, S. J. Zhu, J. H. Hamilton, J. O. Rasmussen, A. V. Ramayya, C. Goodin, K. Li, J. K. Hwang, D. Almehed, S. Frauendorf, V. Dimitrov, Jing-ye Zhang, X. L. Che, Z. Jang, I. Stefanescu, A. Gelberg, G. M. Ter-Akopian, A. V. Daniel, M. A. Stoyer, R. Donangelo, J. D. Cole, N. J. Stone, Phys. Lett. B 670 (2009) 307.

[112] S. Frauendorf, J. Meng, Z. Phys. A 356 (1996) 263.

[113] Y. H. Zhang, S. Q. Zhang, Q. Z. Zhao, S. F. Zhu, H. S. Xu, X. H. Zhou, Y. X. Guo, X. G. Lei, J. Lu, W. X. Huang, Q. B. Gou, H. J. Jin, Z. Liu, Y. X. Luo, X. F. Sun, Y. T. Zhu, X. G. Wu, S. X. Wen, C. X. Yang, Phys. Rev. C 60 (1999) 044311.

[114] C. Liu, Private Communication, January 2018.

[115] J. H. Hamilton, Y. X. Luo, S. J. Zhu, J. O. Rasmussen, A. V. Ramayya, C. Goodin, K. Li, J. K. Hwang, S. Liu, D. Almehed, S. Frauendorf, V. Dimitrov, Jing-ye Zhang, X. L. Che, Z. Jang, I. Stefanescu, A. Gelberg, G. M. Ter-Akopian, A. V. Daniel, I. Y. Lee, H. B. Ding, R. Q. Xu, J. G. Wang, Q. Xu, M. A. Stoyer, R. Donangelo, N. J. Stone, Acta Phys. Pol. B 40 (2009) 523. 
[116] C. Vaman, PhD Dissertation, State University of New York, 2004.

[117] J. Timár, C. Vaman, K. Starosta, D. B. Fossan, T. Koike, D. Sohler, I. Y. Lee, A. O. Macchiavelli, Phys. Rev. C 73 (2006) 011301(R).

[118] T. Suzuki, G. Rainovski, T. Koike, T. Ahn, M. P. Carpenter, A. Costin, M. Danchev, A. Dewald, R. V. F. Janssens, P. Joshi, C. J. Lister, O. Möller, N. Pietralla, T. Shinozuka, J. Timár, R. Wadsworth, C. Vaman, S. Zhu, Phys. Rev. C 78 (2008) 031302(R).

[119] J. Timár, Private Communication, December 2017.

[120] P. Joshi, S. Finnigan, D. B. Fossan, T. Koike, E. S. Paul, G. Rainovski, K. Starosta, C. Vaman, R. Wadsworth, J. Phys. G: Nucl. Part. Phys. 31 (2005) S1895.

[121] P. Joshi, M. P. Carpenter, D. B. Fossan, T. Koike, E. S. Paul, G. Rainovski, K. Starosta, C. Vaman, R. Wadsworth, Phys. Rev. Lett. 98 (2007) 102501.

[122] Y. Zheng, L. H. Zhu, X. G. Wu, C. Y. He, G. S. Li, X. Hao, B. B. Yu, S. H. Yao, B. Zhang, C. Xu, J. G. Wang, L. Gu, Chin. Phys. Lett. 31 (2014) 062101.

[123] N. Rather, P. Datta, S. Chattopadhyay, S. Rajbanshi, A. Goswami, G. H. Bhat, J. A. Sheikh, S. Roy, R. Palit, S. Pal, S. Saha, J. Sethi, S. Biswas, P. Singh, H. C. Jain, Phys. Rev. Lett. 112 (2014) 202503.

[124] R. A. Bark, E. O. Lieder, R. M. Lieder, E. A. Lawrie, J. J. Lawrie, S. P. Bvumbi, N. Y. Kheswa, S. S. Ntshangase, T. E. Madiba, P. L. Masiteng, S. M. Mullins, S. Murray, P. Papka, O. Shirinda, Q. B. Chen, S. Q. Zhang, Z. H. Zhang, P. W. Zhao, C. Xu, J. Meng, D. G. Roux, Z. P. Li, J. Peng, B. Qi, S. Y. Wang, Z. G. Xiao, Int. J. Mod. Phys. E 23 (2014) 1461001.

[125] B. Zhang, L. H. Zhu, H. B. Sun, C. Y. He, X. G. Wu, J. B. Lu, Y. J. Ma, X. Hao, Y. Zheng, B. B. Yu, G. S. Li, S. H. Yao, L. L. Wang, C. Xu, J. G. Wang, L. Gu, Chin. Phys. C 35 (2011) 1009.

[126] S. H. Yao, H. L. Ma, L. H. Zhu, X. G. Wu, C. Y. He, Y. Zheng, B. Zhang, G. S. Li, C. B. Li, S. P. Hu, X. P. Cao, B. B. Yu, C. Xu, Y. Y. Cheng, Phys. Rev. C 89 (2014) 014327.

[127] X. F. Li, Y. J. Ma, Y. Z. Liu, J. B. Lu, G. Y. Zhao, L. C. Yin, R. Meng, Z. L. Zhang, L. J. Wen, X. H. Zhou, Y. X. Guo, X. G. Lei, Z. Liu, J. J. He, Y. Zheng, Chin. Phys. Lett. 19 (2002) 1779.

[128] S. Y. Wang, Y. Z. Liu, T. Komatsubara, Y. J. Ma, Y. H. Zhang, Phys. Rev. C 74 (2006) 017302.

[129] K. Starosta, T. Koike, C. J. Chiara, D. B. Fossan, D. R. LaFosse, A. A. Hecht, C. W. Beausang, M. A. Caprio, J. R. Cooper, R. Krücken, J. R. Novak, N. V. Zamfir, K. E. Zyromski, D. J. Hartley, D. L. Balabanski, Jing-ye Zhang, S. Frauendorf, V. I. Dimitrov, Phys. Rev. Lett. 86 (2001) 971.

[130] L. L. Wang, X. G. Wu, L. H. Zhu, G. S. Li, X. Hao, Y. Zheng, C. Y. He, L. Wang, X. Q. Li, Y. Liu, B. Pan, Z. Y. Li, H. B. Ding, Chin. Phys. C 33 (2009) 173.

[131] X. G. Wu, L. L. Wang, L. H. Zhu, G. S. Li, X. Hao, Y. Zheng, C. Y. He, X. Q. Li, B. Pan, Y. Liu, L. Wang, Y. X. Zhao, Z. Y. Li, H. B. Ding, Plasma Sci. Technol. 14 (2012) 526.

[132] G. Rainovski, E. S. Paul, H. J. Chantler, P. J. Nolan, D. G. Jenkins, R. Wadsworth, P. Raddon, A. Simons, D. B. 
Fossan, T. Koike, K. Starosta, C. Vaman, E. Farnea, A. Gadea, Th. Kröll, G. de Angelis, R. Isocrate, D. Curien, V. I. Dimitrov, J. Phys. G: Nucl. Part. Phys. 29 (2003) 2763.

[133] C. M. Petrache, S. Brant, D. Bazzacco, G. Falconi, E. Farnea, S. Lunardi, V. Paar, Zs. Podolyák, R. Venturelli, D. Vretenar, Nuclear Phys. A 635 (1998) 361.

[134] C. M. Petrache, D. Bazzacco, S. Lunardi, C. Rossi Alvarez, G. de Angelis, M. De Poli, D. Bucurescu, C. A. Ur, P. B. Semmes, R. Wyss, Nuclear Phys. A, 597 (1996) 106.

[135] C. M. Petrache, G. B. Hagemann, I. Hamamoto, K. Starosta, Phys. Rev. Lett. 96 (2006) 112502.

[136] D. Tonev, P. Petkov, D. L. Balabanski, G. de Angelis, A. Gadea, D. R. Napoli, N. Marginean, A. Dewald, P. Pejovic, A. Fitzler, O. Möller, K. O. Zell, S. Brant, S. Frauendorf, D. Bazzacco, S. Lenzi, S. Lunardi, P. Bednarczyk, D. Curien, C. Petrache, Q. Zhong, Y. H. Zhang, Jing-ye Zhang, Int. J. Mod. Phys. E 15 (2006) 1531.

[137] D. Tonev, G. de Angelis, S. Brant, S. Frauendorf, P. Petkov, A. Dewald, F. Dönau, D. L. Balabanski, Q. Zhong, P. Pejovic, D. Bazzacco, P. Bednarczyk, F. Camera, D. Curien, F. Della Vedova, A. Fitzler, A. Gadea, G. Lo Bianco, S. Lenzi, S. Lunardi, N. Marginean, O. Möller, D. R. Napoli, R. Orlandi, E. Sahin, A. Saltarelli, J. Valiente Dobon, K. O. Zell, Jing-ye Zhang, Y. H. Zhang, Phys. Rev. C 76 (2007) 044313.

[138] S. Zhu, U. Garg, B. K. Nayak, S. S. Ghugre, N. S. Pattabiraman, D. B. Fossan, T. Koike, K. Starosta, C. Vaman, R. V. F. Janssens, R. S. Chakrawarthy, M. Whitehead, A. O. Macchiavelli, S. Frauendorf, Phys. Rev. Lett. 91 (2003) 132501.

[139] E. Mergel, C. M. Petrache, G. Lo Bianco, H. Hübel, J. Domscheit, D. Roßbach, G. Schönwaßer, N. Nenoff, A. Neußer, A. Görgen, F. Becker, E. Bouchez, M. Houry, A. Hürstel, Y. Le Coz, R. Lucas, Ch. Theisen, W. Korten, A. Bracco, N. Blasi, F. Camera, S. Leoni, F. Hannachi, A. Lopez-Martens, M. Rejmund, D. Gassmann, P. Reiter, P. G. Thirolf, A. Astier, N. Buforn, M. Meyer, N. Redon, O. Stezowski, Eur. Phys. J. A 15 (2002) 417.

[140] S. Mukhopadhyay, D. Almehed, U. Garg, S. Frauendorf, T. Li, P. V. Madhusudhana Rao, X. Wang, S. S. Ghugre, M. P. Carpenter, S. Gros, A. Hecht, R. V. F. Janssens, F. G. Kondev, T. Lauritsen, D. Seweryniak, S. Zhu, Phys. Rev. C 78 (2008) 034311.

[141] C. W. Beausang, A. A. Hecht, K. E. Zyromski, D. Balabanski, C. J. Barton, M. A. Caprio, R. F. Casten, J. R. Cooper, D. Hartley, R. Krücken, J. R. Novak, N. V. Zamfir, Jing-ye Zhang, F. Dönau, Nuclear Phys. A 682 (2001) $394 \mathrm{c}$.

[142] A. A. Hecht, PhD Dissertation, Yale University, 2004.

[143] P. L. Masiteng, E. A. Lawrie, T. M. Ramashidzha, R. A. Bark, B. G. Carlsson, J. J. Lawrie, R. Lindsay, F. Komati, J. Kau, P. Maine, S. M. Maliage, I. Matamba, S. M. Mullins, S. H. T. Murray, K. P. Mutshena, A. A. Pasternak, I. Ragnarsson, D. G. Roux, J. F. Sharpey-Schafer, O. Shirinda, P. A. Vymers, Phys. Lett. B 719 (2013) 83.

[144] P. L. Masiteng, A. A. Pasternak, E. A. Lawrie, O. Shirinda, J. J. Lawrie, R. A. Bark, S. P. Bvumbi, N. Y. Kheswa, R. Lindsay, E. O. Lieder, R. M. Lieder, T. E. Madiba, S. M. Mullins, S. H. T. Murray, J. Ndayishimye, S. S. Ntshangase, P. Papka, J. F. Sharpey-Schafer, Eur. Phys. J. A 52 (2016) 28.

[145] E. A. Lawrie, P. A. Vymers, J. J. Lawrie, Ch. Vieu, R. A. Bark, R. Lindsay, G. K. Mabala, S. M. Maliage, P. L. 
Masiteng, S. M. Mullins, S. H. T. Murray, I. Ragnarsson, T. M. Ramashidzha, C. Schück, J. F. Sharpey-Schafer, O. Shirinda. Phys. Rev. C 78 (2008) 021305(R). 


\section{Explanation of Tables}

Table 1. Chiral doublet bands.

\begin{tabular}{|c|c|}
\hline \multirow[t]{5}{*}{${ }_{Z}^{A} X_{N}$} & Denotes the specific nuclide with \\
\hline & $X$ chemical symbol \\
\hline & $A$ mass number \\
\hline & $Z$ atomic number \\
\hline & $N$ neutron number \\
\hline
\end{tabular}

A horizontal line connecting a row marks the end of entry for each chiral nucleus. For multiple chiral doublet bands, the pairs of chiral doublet bands are separated by a horizontal line starting from the second column.

$I^{\pi}$

$I$ denotes the level spin in units of $\hbar$ for each band member. $\pi$ denotes the parity $(+$ or -$)$. References for each nucleus are given directly in last column.

$E$ Level energy in units of $\mathrm{keV}$. The number of digits follows the original experimental article. In the energy column, yrast and side denote the two partners of chiral doublet bands. The star marks the level which is the yrast state (the lowest state for given spin) in the nucleus. $B(M 1) / B(E 2) \quad$ The ratio of reduced transition strength in units of $\mu_{N}^{2} / \mathrm{e}^{2} \mathrm{~b}^{2}$ with the uncertainties available in parentheses. The number of digits follow the original experimental article. In the $B(M 1) / B(E 2)$ column, yrast and side denote the two partners of chiral doublet bands. For the nuclei ${ }^{106} \mathrm{Mo},{ }^{98} \mathrm{Tc},{ }^{100} \mathrm{Tc},{ }^{110} \mathrm{Ru},{ }^{112} \mathrm{Ru},{ }^{102} \mathrm{Rh},{ }^{106} \mathrm{Rh},{ }^{111} \mathrm{Rh},{ }^{113} \mathrm{Rh},{ }^{104} \mathrm{Ag},{ }^{126} \mathrm{I},{ }^{128} \mathrm{Cs}$, ${ }^{132} \mathrm{Cs},{ }^{132} \mathrm{La},{ }^{134} \mathrm{La},{ }^{134} \mathrm{Pr},{ }^{136} \mathrm{Nd},{ }^{137} \mathrm{Nd},{ }^{138} \mathrm{Nd},{ }^{188} \mathrm{Ir}$, and ${ }^{198} \mathrm{Tl}$, the $B(M 1) / B(E 2)$ ratios are extracted by the equation (2) in Ref. [113]. For the nuclei ${ }^{104} \mathrm{Rh},{ }^{106} \mathrm{Ag},{ }^{107} \mathrm{Ag},{ }^{124} \mathrm{Cs}$, ${ }^{126} \mathrm{Cs},{ }^{130} \mathrm{Cs},{ }^{135} \mathrm{Nd}$, and ${ }^{194} \mathrm{Tl}$ with $B(M 1)$ and $B(E 2)$ values available, the $B(M 1) / B(E 2)$ ratios are also calculated. For experimental data available, the number of digits follows the significant digits of the error. For the data which extracted from the experimental article, the number of digits is determined by the original data according to the error transfer formula.

Table 2. Chiral doublet bands with $B(M 1)$ and $B(E 2)$ values.
${ }_{Z}^{A} X_{N}$
Same as the explanation in Table 1.
$I^{\pi}$
Same as the explanation in Table 1.
E
Same as the explanation in Table 1.
$B(M 1)$
The magnetic dipole transition strength in units of $\mu_{N}^{2}$ with the uncertainties available in parentheses. The number of digits follows the original experimental article. In the $B(M 1)$ column, yrast and side denote the two partners of chiral doublet bands.
$B(E 2)$

\begin{abstract}
The electric quadrupole transition strength in units of $\mathrm{e}^{2} \mathrm{~b}^{2}$ with the uncertainties available in parentheses. The number of digits follows the original experimental article. In the $B(E 2)$ column, yrast and side denote the two partners of chiral doublet bands.
\end{abstract}




\section{Table 1}

Chiral doublet bands. See page 44 for Explanation of Tables for details.

\begin{tabular}{|c|c|c|c|c|c|c|}
\hline \multirow{2}{*}{ Nuclei } & \multirow{2}{*}{$I^{\pi}(\hbar)$} & \multicolumn{2}{|c|}{$E(\mathrm{keV})$} & \multicolumn{2}{|c|}{$B(M 1) / B(E 2)\left(\mu_{N}^{2} / \mathrm{e}^{2} \mathrm{~b}^{2}\right)$} & \multirow{2}{*}{ References } \\
\hline & & yrast & side & yrast & side & \\
\hline \multirow[t]{26}{*}{${ }_{35}^{78} \mathrm{Br}_{43}$} & $8^{+}$ & $465.7^{*}$ & & & & {$[55,114]$} \\
\hline & $9^{+}$ & $975.3^{*}$ & 1408.6 & & & \\
\hline & $10^{+}$ & $1369.4^{*}$ & 1881.5 & $2.13918\left({ }_{-0.07423}^{+0.07423}\right)$ & & \\
\hline & $11^{+}$ & $1937.8^{*}$ & 2482.8 & $10.56231\left({ }_{-0.92806}^{+0.92806}\right)$ & $6.15398\left({ }_{-0.88693}^{+0.88693}\right)$ & \\
\hline & $12^{+}$ & $2582.5^{*}$ & 2975.9 & $<0.82258$ & $1.6983\left({ }_{-0.40338}^{+0.40338}\right)$ & \\
\hline & $13^{+}$ & $3146.0^{*}$ & 3644.4 & $7.4299\left({ }_{-0.6144}^{+0.6144}\right)$ & & \\
\hline & $14^{+}$ & $4046.2^{*}$ & & $<1.85685$ & & \\
\hline & $15^{+}$ & $4539.0^{*}$ & & $8.02873\left({ }_{-1.56134}^{+1.56134}\right)$ & & \\
\hline & $16^{+}$ & $5600.2^{*}$ & & & & \\
\hline & $17^{+}$ & $6084.6^{*}$ & & & & \\
\hline & $18^{+}$ & 7268.2 & & & & \\
\hline & $19^{+}$ & 7818.4 & & & & \\
\hline & $20^{+}$ & 9132.2 & & & & \\
\hline & $6^{-}$ & 422.6 & 601.1 & & & {$[55,114]$} \\
\hline & $7^{-}$ & 683.4 & 828.6 & & & \\
\hline & $8^{-}$ & 1028.5 & 1190.2 & $0.31614\left({ }_{-0.07277}^{+0.07277}\right)$ & $0.17\left(\begin{array}{l}+0.08 \\
-0.08\end{array}\right)$ & \\
\hline & $9^{-}$ & 1461.1 & 1602.8 & $1.30596\left({ }_{-0.29978}^{+0.29978}\right)$ & $0.65\left({ }_{-0.13}^{+0.13}\right)$ & \\
\hline & $10^{-}$ & 1902.8 & 2026.3 & $<0.39233$ & $<0.2717$ & \\
\hline & $11^{-}$ & 2452.8 & 2666.5 & $2.53562\left({ }_{-1.14277}^{+1.14277}\right)$ & $<0.64594$ & \\
\hline & $12^{-}$ & 3013.8 & 3073.1 & $<1.12632$ & $<1.3865$ & \\
\hline & $13^{-}$ & 3616.8 & & $4.08762\left({ }_{-1.65843}^{+1.65843}\right)$ & & \\
\hline & $14^{-}$ & 4292.0 & 4319.4 & & & \\
\hline & $15^{-}$ & 4919.5 & & & & \\
\hline & $16^{-}$ & 5764.0 & & & & \\
\hline & $17^{-}$ & 6412.3 & & & & \\
\hline & $19^{-}$ & 8045.1 & & & & \\
\hline \multirow[t]{6}{*}{${ }_{35}^{80} \mathrm{Br}_{45}$} & $6^{+}$ & $357.1^{*}$ & & & & {$[74]$} \\
\hline & $7^{+}$ & $447.7^{*}$ & & & & \\
\hline & $8^{+}$ & $616.0^{*}$ & & & & \\
\hline & $9^{+}$ & $1141.6^{*}$ & 1534.8 & $7.6744\left({ }_{-1.6049}^{+1.6049}\right)$ & & \\
\hline & $10^{+}$ & $1588.7^{*}$ & 2002.2 & $1.5662\left({ }_{-0.1989}^{+0.1989}\right)$ & & \\
\hline & $11^{+}$ & $2257.6^{*}$ & 2681.4 & $17.8417\left({ }_{-3.3394}^{+3.3394}\right)$ & $7.3\left(\begin{array}{l}+1.8 \\
-1.8\end{array}\right)$ & \\
\hline
\end{tabular}


Table 1 (continued)

\begin{tabular}{|c|c|c|c|c|c|c|}
\hline \multirow{2}{*}{ Nuclei } & \multirow{2}{*}{$I^{\pi}(\hbar)$} & \multicolumn{2}{|c|}{$E(\mathrm{keV})$} & \multicolumn{2}{|c|}{$B(M 1) / B(E 2)\left(\mu_{N}^{2} / \mathrm{e}^{2} \mathrm{~b}^{2}\right)$} & \multirow{2}{*}{ References } \\
\hline & & yrast & side & yrast & side & \\
\hline & $12^{+}$ & $2945.4^{*}$ & 3212.9 & $1.9518\left(\begin{array}{l}+0.2036 \\
-0.2036\end{array}\right)$ & $5.3\left(\begin{array}{l}+1.3 \\
-1.3\end{array}\right)$ & \\
\hline & $13^{+}$ & $3658.7^{*}$ & 3972.9 & $6.206\left(\begin{array}{l}+1.8574 \\
-1.8574\end{array}\right)$ & & \\
\hline & $14^{+}$ & $4451.8^{*}$ & & $0.2253\left({ }_{-0.0484}^{+0.0484}\right)$ & & \\
\hline & $15^{+}$ & 5195.2 & & & & \\
\hline & $16^{+}$ & 5936.5 & & & & \\
\hline \multirow[t]{11}{*}{${ }_{42}^{106} \mathrm{Mo}_{64}$} & $4^{-}$ & & 1937.0 & & & {$[75,110]$} \\
\hline & $5^{-}$ & 1952.4 & 2090.6 & & & \\
\hline & $6^{-}$ & 2142.9 & 2276.5 & & & \\
\hline & $7^{-}$ & 2369.5 & 2499.0 & 0.58 & 0.22 & \\
\hline & $8^{-}$ & 2630.1 & 2746.6 & 0.40 & 0.16 & \\
\hline & $9^{-}$ & 2922.3 & 3041.7 & 0.25 & 0.15 & \\
\hline & $10^{-}$ & 3239.5 & 3349.8 & 0.22 & 0.28 & \\
\hline & $11^{-}$ & 3592.8 & 3707.7 & $<0.55$ & 0.42 & \\
\hline & $12^{-}$ & 3946.4 & 4049.4 & $<0.58$ & $<0.49$ & \\
\hline & $13^{-}$ & 4372.7 & & & & \\
\hline & $14^{-}$ & 4753.2 & & & & \\
\hline \multirow[t]{7}{*}{${ }_{43}^{98} \mathrm{Tc}_{55}$} & $9^{-}$ & 1166.2 & & & & {$[76]$} \\
\hline & $10^{-}$ & 1582.5 & 1920.6 & 9.7 & & \\
\hline & $11^{-}$ & $1851.4^{*}$ & 2368.8 & 7.2 & & \\
\hline & $12^{-}$ & $2303.9^{*}$ & 2671.1 & 5.9 & 3.0 & \\
\hline & $13^{-}$ & $2677.4^{*}$ & 3266.3 & 2.4 & $<1.9$ & \\
\hline & $14^{-}$ & $3130.2^{*}$ & & 1.9 & & \\
\hline & $15^{-}$ & 3724.7 & & 0.9 & & \\
\hline \multirow[t]{9}{*}{${ }_{43}^{100} \mathrm{Tc}_{57}$} & $9^{-}$ & $778^{*}$ & & & & {$[77]$} \\
\hline & $10^{-}$ & $1155^{*}$ & 1583 & & & \\
\hline & $11^{-}$ & $1406^{*}$ & 2055 & $6.55\left({ }_{-0.04}^{+0.04}\right)$ & & \\
\hline & $12^{-}$ & $1840^{*}$ & 2394 & $10.34\left({ }_{-0.17}^{+0.17}\right)$ & $12.93\left({ }_{-4.14}^{+4.14}\right)$ & \\
\hline & $13^{-}$ & $2238^{*}$ & 2802 & $2.67\left(\begin{array}{l}+0.02 \\
-0.02\end{array}\right)$ & & \\
\hline & $14^{-}$ & $2693^{*}$ & 3273 & $6.41\left(\begin{array}{l}+0.46 \\
-0.46\end{array}\right)$ & & \\
\hline & $15^{-}$ & $3234^{*}$ & 3693 & $1.71\left(\begin{array}{l}+0.18 \\
-0.18\end{array}\right)$ & & \\
\hline & $16^{-}$ & $3713^{*}$ & & $2.99\left(\begin{array}{c}+0.32 \\
-0.32\end{array}\right)$ & & \\
\hline & $17^{-}$ & $4357^{*}$ & & $3.13\left(\begin{array}{l}+1.74 \\
-1.74\end{array}\right)$ & & \\
\hline \multirow[t]{2}{*}{${ }_{44}^{110} \mathrm{Ru}_{66}$} & $4^{-}$ & & 2016.2 & & & {$[75,78,111,115]$} \\
\hline & $5^{-}$ & & 2145.3 & & & \\
\hline
\end{tabular}


Table 1 (continued)

\begin{tabular}{|c|c|c|c|c|c|c|}
\hline \multirow{2}{*}{ Nuclei } & \multirow{2}{*}{$I^{\pi}(\hbar)$} & \multicolumn{2}{|c|}{$E(\mathrm{keV})$} & \multicolumn{2}{|c|}{$B(M 1) / B(E 2)\left(\mu_{N}^{2} / \mathrm{e}^{2} \mathrm{~b}^{2}\right)$} & \multirow{2}{*}{ References } \\
\hline & & yrast & side & yrast & side & \\
\hline & $6^{-}$ & 2242.9 & 2328.0 & & & \\
\hline & $7^{-}$ & 2426.5 & 2516.7 & & & \\
\hline & $8^{-}$ & 2637.4 & 2764.7 & 0.38 & 0.30 & \\
\hline & $9^{-}$ & 2892.7 & 3041.4 & 0.40 & 0.24 & \\
\hline & $10^{-}$ & 3175.3 & 3337.1 & 0.29 & 0.48 & \\
\hline & $11^{-}$ & $3485.3^{*}$ & 3689.9 & 0.42 & 0.23 & \\
\hline & $12^{-}$ & 3818.5 & 4038.8 & 0.32 & 0.63 & \\
\hline & $13^{-}$ & $4195.5^{*}$ & 4446.3 & $<0.77$ & & \\
\hline & $14^{-}$ & 4566.5 & 4874.1 & & & \\
\hline & $15^{-}$ & $5010.8^{*}$ & 5302.5 & & & \\
\hline & $16^{-}$ & 5412.8 & & & & \\
\hline \multirow[t]{11}{*}{${ }_{44}^{112} \mathrm{Ru}_{68}$} & $5^{-}$ & 2003.2 & & & & {$[75,78,111,115]$} \\
\hline & $6^{-}$ & 2230.2 & 2334.2 & & & \\
\hline & $7^{-}$ & 2489.2 & 2574.3 & & & \\
\hline & $8^{-}$ & 2771.7 & 2829.2 & 0.50 & & \\
\hline & $9^{-}$ & 3076.5 & 3094.1 & 0.29 & 0.20 & \\
\hline & $10^{-}$ & 3420.8 & 3379.8 & 0.40 & 0.38 & \\
\hline & $11^{-}$ & 3768.3 & 3711.5 & 0.67 & 0.34 & \\
\hline & $12^{-}$ & 4198.8 & 4032.5 & 0.71 & $<0.91$ & \\
\hline & $13^{-}$ & 4561.7 & 4428.3 & & & \\
\hline & $14^{-}$ & 5072.9 & 4769.6 & & & \\
\hline & $15^{-}$ & & 5227.8 & & & \\
\hline \multirow[t]{11}{*}{${ }_{45}^{102} \mathrm{Rh}_{57}$} & $8^{-}$ & 78 & & & & {$[79,116]$} \\
\hline & $9^{-}$ & 224 & & & & \\
\hline & $10^{-}$ & $587^{*}$ & 1048 & $6.32\left({ }_{-0.53}^{+0.53}\right)$ & & \\
\hline & $11^{-}$ & $893^{*}$ & 1500 & $11.42\left(\begin{array}{l}+0.79 \\
-0.79\end{array}\right)$ & & \\
\hline & $12^{-}$ & $1355^{*}$ & 1859 & $11.27\left(\left(_{-0.83}^{+0.83}\right)\right.$ & $4.24\left(\begin{array}{l}+0.63 \\
-0.63\end{array}\right)$ & \\
\hline & $13^{-}$ & $1793^{*}$ & 2323 & $7.37\left(\begin{array}{l}+0.50 \\
-0.50\end{array}\right)$ & $6.94\left(\begin{array}{l}+2.44 \\
-2.44\end{array}\right)$ & \\
\hline & $14^{-}$ & $2281^{*}$ & & $15.61\left({ }_{-1.37}^{+1.37}\right)$ & $22.82\left(\begin{array}{l}+5.48 \\
-5.48\end{array}\right)$ & \\
\hline & $15^{-}$ & $2809^{*}$ & & $7.16\left(\begin{array}{l}+0.70 \\
-0.70\end{array}\right)$ & & \\
\hline & $16^{-}$ & $3337^{*}$ & & $10.85\left({ }_{-1.18}^{+1.18}\right)$ & & \\
\hline & $17^{-}$ & $3939^{*}$ & & $7.87\left({ }_{-0.79}^{+0.79}\right)$ & & \\
\hline & $18^{-}$ & $4544^{*}$ & & $10.49\left(\begin{array}{c}+1.50 \\
-1.50\end{array}\right)$ & & \\
\hline${ }_{45}^{103} \mathrm{Rh}_{58}$ & $10.5^{+}$ & 3238 & & & & {$[54,117-119]$} \\
\hline
\end{tabular}


Table 1 (continued)

\begin{tabular}{|c|c|c|c|c|c|c|}
\hline \multirow{2}{*}{ Nuclei } & \multirow{2}{*}{$I^{\pi}(\hbar)$} & \multicolumn{2}{|c|}{$E(\mathrm{keV})$} & \multicolumn{2}{|c|}{$B(M 1) / B(E 2)\left(\mu_{N}^{2} / \mathrm{e}^{2} \mathrm{~b}^{2}\right)$} & \multirow{2}{*}{ References } \\
\hline & & yrast & side & yrast & side & \\
\hline & $11.5^{+}$ & 3357 & & & & \\
\hline & $12.5^{+}$ & 3591 & & $23.9\left({ }_{-8.3}^{+8.3}\right)$ & & \\
\hline & $13.5^{+}$ & 3899 & 4445 & $14.2\left(\begin{array}{c}+1.8 \\
-1.8\end{array}\right)$ & & \\
\hline & $14.5^{+}$ & 4281 & 4789 & $11.4\left({ }_{-1.6}^{+1.6}\right)$ & & \\
\hline & $15.5^{+}$ & 4665 & 5166 & $12.2\left(\begin{array}{l}+2.1 \\
-2.1\end{array}\right)$ & $12.1\left(\begin{array}{l}+2.9 \\
-2.9\end{array}\right)$ & \\
\hline & $16.5^{+}$ & 5156 & 5616 & $13.3\left({ }_{-1.7}^{+1.7}\right)$ & $10.3\left({ }_{-3.0}^{+3.0}\right)$ & \\
\hline & $17.5^{+}$ & 5622 & 6062 & $11.8\left(\begin{array}{l}+1.7 \\
-1.7\end{array}\right)$ & $5.7\left({ }_{-1.3}^{+1.3}\right)$ & \\
\hline & $18.5^{+}$ & 6163 & 6528 & $15.1\left(\begin{array}{l}+3.2 \\
-3.2\end{array}\right)$ & $23.9\left({ }_{-12.7}^{+12.7}\right)$ & \\
\hline & $19.5^{+}$ & 6706 & 7074 & $15.0\left(\begin{array}{l}+5.2 \\
-5.2\end{array}\right)$ & $9.0\left(\begin{array}{l}+5.0 \\
-5.0\end{array}\right)$ & \\
\hline & $20.5^{+}$ & 7317 & & $22.8\left(\begin{array}{l}+9.8 \\
-9.8\end{array}\right)$ & & \\
\hline & $21.5^{+}$ & 7952 & & $17.4\left(\begin{array}{l}+8.5 \\
-8.5\end{array}\right)$ & & \\
\hline & $6.5^{-}$ & 2033 & & & & {$[54,117-119]$} \\
\hline & $7.5^{-}$ & 2219 & & & & \\
\hline & $8.5^{-}$ & 2343 & & $40.6\left(\begin{array}{l}+7.3 \\
-7.3\end{array}\right)$ & & \\
\hline & $9.5^{-}$ & 2538 & 2744 & $25.0\left(\begin{array}{l}+3.3 \\
-3.3\end{array}\right)$ & & \\
\hline & $10.5^{-}$ & 2751 & 2934 & $37.1\left(\begin{array}{l}+5.8 \\
-5.8\end{array}\right)$ & & \\
\hline & $11.5^{-}$ & $3011^{*}$ & 3273 & $16.8\left(\begin{array}{l}+2.0 \\
-2.0\end{array}\right)$ & & \\
\hline & $12.5^{-}$ & $3327^{*}$ & 3615 & $16.8\left(\begin{array}{l}+3.6 \\
-3.6\end{array}\right)$ & $21.1\left(\begin{array}{l}+2.5 \\
-2.5\end{array}\right)$ & \\
\hline & $13.5^{-}$ & $3769^{*}$ & 4080 & $12.9\left(\begin{array}{l}+2.2 \\
-2.2\end{array}\right)$ & $12.6\left({ }_{-1.5}^{+1.5}\right)$ & \\
\hline & $14.5^{-}$ & 4195 & 4559 & $10.0\left(\begin{array}{l}+2.4 \\
-2.4\end{array}\right)$ & $10.2\left(\begin{array}{l}+1.5 \\
-1.5\end{array}\right)$ & \\
\hline & $15.5^{-}$ & 4763 & 5091 & $13.4\left(\begin{array}{l}+3.0 \\
-3.0\end{array}\right)$ & $5.4\left(\begin{array}{l}+1.5 \\
-1.5\end{array}\right)$ & \\
\hline & $16.5^{-}$ & 5297 & 5685 & $6.7\left(\begin{array}{l}+1.0 \\
-1.0\end{array}\right)$ & $6.8\left(\begin{array}{l}-3.9 \\
-3.9\end{array}\right)$ & \\
\hline & $17.5^{-}$ & 5923 & & $11.3\left(\begin{array}{l}+2.9 \\
-2.9\end{array}\right)$ & & \\
\hline & $18.5^{-}$ & 6586 & & $4.3\left({ }_{-1.5}^{+1.5}\right)$ & & \\
\hline & $19.5^{-}$ & 7186 & & $12.4\left(\begin{array}{l}+5.4 \\
-5.4\end{array}\right)$ & & \\
\hline & $6.5^{-}$ & 2228 & & & & {$[54,117-119]$} \\
\hline & $7.5^{-}$ & 2366 & 2443 & & & \\
\hline & $8.5^{-}$ & 2520 & 2643 & & & \\
\hline & $9.5^{-}$ & 2699 & 2871 & $30.8\left({ }_{-4.8}^{+4.8}\right)$ & $10.3\left(\begin{array}{l}+3.5 \\
-3.5\end{array}\right)$ & \\
\hline & $10.5^{-}$ & 2915 & 3090 & $18.2\left({ }_{-2.0}^{+2.0}\right)$ & $33.1\left({ }_{-13.4}^{+13.4}\right)$ & \\
\hline & $11.5^{-}$ & 3227 & 3416 & $28.2\left(\begin{array}{l}+9.7 \\
-9.7\end{array}\right)$ & $15.2\left(\begin{array}{l}+5.1 \\
-5.1\end{array}\right)$ & \\
\hline & $12.5^{-}$ & 3668 & 3778 & $17.7\left(\begin{array}{l}+2.5 \\
-2.5\end{array}\right)$ & $13.7\left({ }_{-3.0}^{+3.0}\right)$ & \\
\hline & $13.5^{-}$ & 4106 & 4210 & $3.8\left(\begin{array}{l}+0.5 \\
-0.5\end{array}\right)$ & $10.2\left(\begin{array}{l}+2.6 \\
-2.6\end{array}\right)$ & \\
\hline & $14.5^{-}$ & 4606 & 4659 & $5.3\left(\begin{array}{l}+0.5 \\
-0.5\end{array}\right)$ & $11.9\left(\begin{array}{l}+3.4 \\
-3.4\end{array}\right)$ & \\
\hline
\end{tabular}


Table 1 (continued)

\begin{tabular}{|c|c|c|c|c|c|c|}
\hline \multirow{2}{*}{ Nuclei } & \multirow{2}{*}{$I^{\pi}(\hbar)$} & \multicolumn{2}{|c|}{$E(\mathrm{keV})$} & \multicolumn{2}{|c|}{$B(M 1) / B(E 2)\left(\mu_{N}^{2} / \mathrm{e}^{2} \mathrm{~b}^{2}\right)$} & \multirow{2}{*}{ References } \\
\hline & & yrast & side & yrast & side & \\
\hline & $15.5^{-}$ & 5061 & 5059 & $2.7\left(\begin{array}{l}+0.6 \\
-0.6\end{array}\right)$ & & \\
\hline & $16.5^{-}$ & 5577 & & $4.3\left(\begin{array}{l}+1.0 \\
-1.0\end{array}\right)$ & & \\
\hline & $17.5^{-}$ & 6143 & & $6.8\left(\begin{array}{l}+3.5 \\
-3.5\end{array}\right)$ & & \\
\hline \multirow[t]{9}{*}{${ }_{45}^{104} \mathrm{Rh}_{59}$} & $9^{-}$ & $483^{*}$ & & & & {$[80,118]$} \\
\hline & $10^{-}$ & $840^{*}$ & 1228 & $15.079\left(\begin{array}{l}+2.643 \\
-2.643\end{array}\right)$ & & \\
\hline & $11^{-}$ & $1168^{*}$ & 1582 & $9.785\left(\begin{array}{l}+1.483 \\
-1.483\end{array}\right)$ & & \\
\hline & $12^{-}$ & $1636^{*}$ & 1971 & $11.282\left(\begin{array}{l}+1.384 \\
-1.384\end{array}\right)$ & & \\
\hline & $13^{-}$ & $2111^{*}$ & 2370 & $6.289\left(\begin{array}{l}+0.646 \\
-0.646\end{array}\right)$ & & \\
\hline & $14^{-}$ & $2639^{*}$ & 2834 & & & \\
\hline & $15^{-}$ & $3229^{*}$ & 3320 & & & \\
\hline & $16^{-}$ & $3800^{*}$ & 3876 & & & \\
\hline & $17^{-}$ & 4406 & 4406 & & & \\
\hline \multirow[t]{20}{*}{${ }_{45}^{105} \mathrm{Rh}_{60}$} & $11.5^{+}$ & $2982^{*}$ & & & & {$[57,58,119]$} \\
\hline & $12.5^{+}$ & $3198^{*}$ & & & & \\
\hline & $13.5^{+}$ & $3478^{*}$ & 4003 & $9.9\left(\begin{array}{c}+1.8 \\
-1.8\end{array}\right)$ & & \\
\hline & $14.5^{+}$ & $3839^{*}$ & 4299 & $15.6\left({ }_{-3.6}^{+3.6}\right)$ & & \\
\hline & $15.5^{+}$ & $4215^{*}$ & 4690 & $13.6\left(\begin{array}{l}+3.2 \\
-3.2\end{array}\right)$ & $8.2\left({ }_{-2.7}^{+2.7}\right)$ & \\
\hline & $16.5^{+}$ & $4702^{*}$ & 5081 & $14.4\left(\begin{array}{l}+2.9 \\
-2.9\end{array}\right)$ & $11.1\left(\begin{array}{l}+3.2 \\
-3.2\end{array}\right)$ & \\
\hline & $17.5^{+}$ & 5184 & 5525 & $13.5\left(\begin{array}{l}+2.8 \\
-2.8\end{array}\right)$ & $8.6\left(\begin{array}{l}+2.5 \\
-2.5\end{array}\right)$ & \\
\hline & $18.5^{+}$ & 5764 & 6020 & $13.4\left(\begin{array}{l}+2.9 \\
-2.9\end{array}\right)$ & $9.1\left(\begin{array}{c}+2.7 \\
-2.7\end{array}\right)$ & \\
\hline & $19.5^{+}$ & 6345 & 6566 & $11.3\left(\begin{array}{l}+2.2 \\
-2.2\end{array}\right)$ & $7.5\left({ }_{-2.6}^{+2.6}\right)$ & \\
\hline & $20.5^{+}$ & 7038 & 7156 & $10.8\left(\begin{array}{l}+2.9 \\
-2.9\end{array}\right)$ & $8.2\left({ }_{-4.4}^{+4.4}\right)$ & \\
\hline & $21.5^{+}$ & 7713 & & $8.4\left(\begin{array}{l}+3.0 \\
-3.0\end{array}\right)$ & & \\
\hline & $22.5^{+}$ & 8467 & 8524 & $9.6\left(\begin{array}{l}+5.4 \\
-5.4\end{array}\right)$ & & \\
\hline & $23.5^{+}$ & 9213 & & & & \\
\hline & $7.5^{-}$ & & 2417 & & & {$[57]$} \\
\hline & $8.5^{-}$ & 2478 & 2512 & & & \\
\hline & $9.5^{-}$ & 2670 & 2645 & & & \\
\hline & $10.5^{-}$ & 2915 & 2824 & & & \\
\hline & $11.5^{-}$ & 3268 & 3077 & & & \\
\hline & $12.5^{-}$ & 3669 & 3469 & & & \\
\hline & $13.5^{-}$ & 4094 & & & & \\
\hline \multirow[t]{2}{*}{${ }_{45}^{106} \mathrm{Rh}_{61}$} & $9^{-}$ & $428^{*}$ & & & & {$[81]$} \\
\hline & $10^{-}$ & $761^{*}$ & 1053 & $8.87\left(\begin{array}{l}+2.42 \\
-2.42\end{array}\right)$ & & \\
\hline
\end{tabular}


Table 1 (continued)

\begin{tabular}{|c|c|c|c|c|c|c|}
\hline \multirow{2}{*}{ Nuclei } & \multirow{2}{*}{$I^{\pi}(\hbar)$} & \multicolumn{2}{|c|}{$E(\mathrm{keV})$} & \multicolumn{2}{|c|}{$B(M 1) / B(E 2)\left(\mu_{N}^{2} / \mathrm{e}^{2} \mathrm{~b}^{2}\right)$} & \multirow{2}{*}{ References } \\
\hline & & yrast & side & yrast & side & \\
\hline & $11^{-}$ & $1071^{*}$ & 1410 & $5.66\left(\begin{array}{c}+0.30 \\
-0.30\end{array}\right)$ & & \\
\hline & $12^{-}$ & $1488^{*}$ & 1787 & $8.13\left(\begin{array}{l}+0.92 \\
-0.92\end{array}\right)$ & $59.10\left(\begin{array}{l}+61.39 \\
-61.39\end{array}\right)$ & \\
\hline & $13^{-}$ & $1905^{*}$ & 2258 & $4.66\left(\begin{array}{l}+0.36 \\
-0.36\end{array}\right)$ & $2.23\left(\begin{array}{c}+0.82 \\
-0.82\end{array}\right)$ & \\
\hline & $14^{-}$ & $2359^{*}$ & 2730 & $6.92\left(\begin{array}{c}+0.58 \\
-0.58\end{array}\right)$ & $10.11\left({ }_{-6.10}^{+6.10}\right)$ & \\
\hline & $15^{-}$ & $2859^{*}$ & 3214 & $4.52\left(\begin{array}{c}+0.45 \\
-0.45\end{array}\right)$ & $5.68\left(\begin{array}{l}+2.94 \\
-2.94\end{array}\right)$ & \\
\hline & $16^{-}$ & $3323^{*}$ & 3708 & $5.93\left(\begin{array}{c}+0.60 \\
-0.60\end{array}\right)$ & $6.06\left(\begin{array}{l}+9.90 \\
-9.90\end{array}\right)$ & \\
\hline & $17^{-}$ & $3864^{*}$ & & $4.44\left(\begin{array}{c}+0.91 \\
-0.91\end{array}\right)$ & & \\
\hline & $18^{-}$ & $4320^{*}$ & & $7.27\left(\begin{array}{l}+1.87 \\
-1.87\end{array}\right)$ & & \\
\hline & $19^{-}$ & $4861^{*}$ & & $3.77\left(\begin{array}{l}+1.59 \\
-1.59\end{array}\right)$ & & \\
\hline & $20^{-}$ & $5363^{*}$ & & $3.85\left(\begin{array}{l}+1.01 \\
-1.01\end{array}\right)$ & & \\
\hline & $21^{-}$ & $5948^{*}$ & & $4.57\left(\begin{array}{l}+2.28 \\
-2.28\end{array}\right)$ & & \\
\hline & $22^{-}$ & $6533^{*}$ & & $4.64\left(\begin{array}{l}+2.59 \\
-2.59\end{array}\right)$ & & \\
\hline \multirow[t]{7}{*}{${ }_{45}^{111} \mathrm{Rh}_{66}$} & $10.5^{+}$ & $2112.7^{*}$ & & & & {$[82]$} \\
\hline & $11.5^{+}$ & $2355.4^{*}$ & 2733.2 & & 2.39 & \\
\hline & $12.5^{+}$ & $2650.8^{*}$ & 2984.5 & 4.79 & 8.25 & \\
\hline & $13.5^{+}$ & $2964.4^{*}$ & 3272.3 & 5.74 & & \\
\hline & $14.5^{+}$ & $3325.4^{*}$ & 3523.9 & 4.92 & 4.09 & \\
\hline & $15.5^{+}$ & $3742.5^{*}$ & 3933.4 & & & \\
\hline & $16.5^{+}$ & & $4249.3^{*}$ & & & \\
\hline \multirow[t]{8}{*}{${ }_{45}^{113} \mathrm{Rh}_{68}$} & $9.5^{+}$ & $1775.5^{*}$ & & 3.27 & & {$[82]$} \\
\hline & $10.5^{+}$ & $2038.0^{*}$ & 2133.2 & 3.23 & & \\
\hline & $11.5^{+}$ & 2470.3 & $2446.5^{*}$ & 1.90 & & \\
\hline & $12.5^{+}$ & $2723.3^{*}$ & 2776.9 & 4.47 & 3.84 & \\
\hline & $13.5^{+}$ & $3090.9^{*}$ & 3133.0 & 3.23 & & \\
\hline & $14.5^{+}$ & $3334.8^{*}$ & & & & \\
\hline & $15.5^{+}$ & $3770.2^{*}$ & & & & \\
\hline & $16.5^{+}$ & $4006.0^{*}$ & & & & \\
\hline \multirow[t]{7}{*}{${ }_{47}^{104} \mathrm{Ag}_{57}$} & $8^{-}$ & 1077 & & & & {$[83]$} \\
\hline & $9^{-}$ & 1253 & & & & \\
\hline & $10^{-}$ & $1599^{*}$ & 2212 & & & \\
\hline & $11^{-}$ & $1932^{*}$ & 2711 & $13.3\left(\begin{array}{l}+1.8 \\
-1.8\end{array}\right)$ & & \\
\hline & $12^{-}$ & $2376^{*}$ & 3040 & $11.9\left({ }_{-2.7}^{+2.7}\right)$ & $13.0\left({ }_{-5.0}^{+5.0}\right)$ & \\
\hline & $13^{-}$ & $2820^{*}$ & 3351 & $12.2\left(\begin{array}{l}+3.2 \\
-3.2\end{array}\right)$ & $18.4\left(\begin{array}{l}+8.3 \\
-8.3\end{array}\right)$ & \\
\hline & $14^{-}$ & $3301^{*}$ & 3648 & $14.1\left(\begin{array}{l}+3.8 \\
-3.8\end{array}\right)$ & & \\
\hline
\end{tabular}


Table 1 (continued)

\begin{tabular}{|c|c|c|c|c|c|c|}
\hline \multirow{2}{*}{ Nuclei } & \multirow{2}{*}{$I^{\pi}(\hbar)$} & \multicolumn{2}{|c|}{$E(\mathrm{keV})$} & \multicolumn{2}{|c|}{$B(M 1) / B(E 2)\left(\mu_{N}^{2} / \mathrm{e}^{2} \mathrm{~b}^{2}\right)$} & \multirow{2}{*}{ References } \\
\hline & & yrast & side & yrast & side & \\
\hline & $15^{-}$ & $3809^{*}$ & 4097 & $14.4\left(\begin{array}{l}+3.2 \\
-3.2\end{array}\right)$ & & \\
\hline & $16^{-}$ & $4329^{*}$ & 4625 & $14.1\left(\begin{array}{l}+2.2 \\
-2.2\end{array}\right)$ & $17.2\left(\begin{array}{l}+6.8 \\
-6.8\end{array}\right)$ & \\
\hline & $17^{-}$ & $4901^{*}$ & & $11.7\left(\begin{array}{l}+3.0 \\
-3.0\end{array}\right)$ & & \\
\hline & $18^{-}$ & $5529^{*}$ & & $16.3\left(\begin{array}{l}+5.7 \\
-5.7\end{array}\right)$ & & \\
\hline & $19^{-}$ & 6133 & & & & \\
\hline \multirow[t]{9}{*}{${ }_{47}^{105} \mathrm{Ag}_{58}$} & $7.5^{-}$ & & 2622.2 & & & {$[84]$} \\
\hline & $8.5^{-}$ & & 2775.2 & & & \\
\hline & $9.5^{-}$ & 2908.2 & 2944.2 & & & \\
\hline & $10.5^{-}$ & 3102.2 & 3177.2 & & & \\
\hline & $11.5^{-}$ & 3409.2 & 3481.2 & & & \\
\hline & $12.5^{-}$ & 3786.2 & 3867.2 & & $17.0\left(\begin{array}{l}+4.4 \\
-4.4\end{array}\right)$ & \\
\hline & $13.5^{-}$ & 4250.2 & 4314.2 & $8.0\left(\begin{array}{l}+1.6 \\
-1.6\end{array}\right)$ & $10.7\left(\begin{array}{c}+1.9 \\
-1.9\end{array}\right)$ & \\
\hline & $14.5^{-}$ & 4719.2 & 4797.2 & $14.2\left(\begin{array}{c}+3.1 \\
-3.1\end{array}\right)$ & $12.1\left({ }_{-2.5}^{+2.5}\right)$ & \\
\hline & $15.5^{-}$ & 5227.2 & 5335.2 & $6.5\left(\begin{array}{l}+2.0 \\
-2.0\end{array}\right)$ & $8.2\left(\begin{array}{l}+4.3 \\
-4.3\end{array}\right)$ & \\
\hline \multirow[t]{13}{*}{${ }_{47}^{106} \mathrm{Ag}_{59}$} & $10^{-}$ & 2271.7 & 3203.8 & & & {$[85,120-124]$} \\
\hline & $11^{-}$ & 2441.4 & 3423.5 & & & \\
\hline & $12^{-}$ & 2660.4 & 3676.0 & & & \\
\hline & $13^{-}$ & 2930.2 & 3941.6 & & & \\
\hline & $14^{-}$ & 3256.7 & 4263.7 & $13.350\left(\begin{array}{l}+6.980 \\
-6.980\end{array}\right)$ & & \\
\hline & $15^{-}$ & 3686.1 & 4636.6 & $9.132\left({ }_{-2.633}^{+2.633}\right)$ & $11.675\left(\begin{array}{l}+4.845 \\
-4.845\end{array}\right)$ & \\
\hline & $16^{-}$ & $4223.3^{*}$ & 5051.7 & $5.444\left(\begin{array}{l}+1.273 \\
-1.273\end{array}\right)$ & $8.797\left({ }_{-2.337}^{+2.337}\right)$ & \\
\hline & $17^{-}$ & $4742.6^{*}$ & 5561.2 & $4.584\left(\begin{array}{l}+1.313 \\
-1.313\end{array}\right)$ & $6.863\left({ }_{-2.269}^{+2.269}\right)$ & \\
\hline & $18^{-}$ & $5414.9^{*}$ & 6065.8 & $7.471\left({ }_{-4.078}^{+4.078}\right)$ & $6.391\left({ }_{-2.115}^{+2.115}\right)$ & \\
\hline & $19^{-}$ & $6026.8^{*}$ & 6691.0 & $5.686\left(\begin{array}{l}+1.459 \\
-1.459\end{array}\right)$ & $5.434\left({ }_{-2.013}^{+2.013}\right)$ & \\
\hline & $20^{-}$ & $6760.7^{*}$ & 7276.7 & $6.721\left({ }_{-2.400}^{+2.400}\right)$ & $6.544\left(\begin{array}{l}+3.018 \\
-3.018\end{array}\right)$ & \\
\hline & $21^{-}$ & 7531.7 & 7945.6 & $1.857\left({ }_{-1.092}^{+1.092}\right)$ & & \\
\hline & $22^{-}$ & 8192.5 & & & & \\
\hline \multirow[t]{7}{*}{${ }_{47}^{107} \mathrm{Ag}_{60}$} & $10.5^{-}$ & $2620.8^{*}$ & 2901.6 & & & {$[60,125,126]$} \\
\hline & $11.5^{-}$ & $2928.7^{*}$ & 3091.8 & $6.94\left(\begin{array}{l}+3.06 \\
-3.06\end{array}\right)$ & & \\
\hline & $12.5^{-}$ & $3338.7^{*}$ & 3392.0 & $9.11\left(\begin{array}{l}+2.85 \\
-2.85\end{array}\right)$ & & \\
\hline & $13.5^{-}$ & 3800.0 & 3897.5 & $10.63\left({ }_{-3.40}^{+3.40}\right)$ & & \\
\hline & $14.5^{-}$ & 4270.0 & 4349.6 & 6.50 & & \\
\hline & $15.5^{-}$ & 4878.8 & 4932.0 & & & \\
\hline & $16.5^{-}$ & 5437.3 & & & & \\
\hline
\end{tabular}


Table 1 (continued)

\begin{tabular}{|c|c|c|c|c|c|c|}
\hline \multirow{2}{*}{ Nuclei } & \multirow{2}{*}{$I^{\pi}(\hbar)$} & \multicolumn{2}{|c|}{$E(\mathrm{keV})$} & \multicolumn{2}{|c|}{$B(M 1) / B(E 2)\left(\mu_{N}^{2} / \mathrm{e}^{2} \mathrm{~b}^{2}\right)$} & \multirow{2}{*}{ References } \\
\hline & & yrast & side & yrast & side & \\
\hline & $11.5^{+}$ & 3334.8 & & & & {$[60,61,125,126]$} \\
\hline & $12.5^{+}$ & 3556.7 & & $34.67\left({ }_{-22.29}^{+22.29}\right)$ & & \\
\hline & $13.5^{+}$ & 3851.3 & & $11.74\left(\begin{array}{l}+3.65 \\
-3.65\end{array}\right)$ & & \\
\hline & $14.5^{+}$ & $4230.0^{*}$ & 4841.7 & $12.00\left({ }_{-3.12}^{+3.12}\right)$ & & \\
\hline & $15.5^{+}$ & $4626.4^{*}$ & 5131.1 & $13.57\left({ }_{-3.61}^{+3.61}\right)$ & & \\
\hline & $16.5^{+}$ & $5120.5^{*}$ & 5448.9 & $8.26\left(\begin{array}{l}+2.77 \\
-2.77\end{array}\right)$ & & \\
\hline & $17.5^{+}$ & $5621.5^{*}$ & 5818.4 & 7.81 & & \\
\hline & $18.5^{+}$ & $6192.8^{*}$ & 6250.2 & & & \\
\hline & $19.5^{+}$ & 6785.9 & $6761.3^{*}$ & & & \\
\hline & $20.5^{+}$ & 7441.1 & $7315.5^{*}$ & & & \\
\hline & $21.5^{+}$ & & $7919.9^{*}$ & & & \\
\hline & $22.5^{+}$ & & $8591.2^{*}$ & & & \\
\hline \multirow[t]{9}{*}{${ }_{53}^{118} \mathrm{I}_{65}$} & $7^{-}$ & $0^{*}$ & & & & {$[86]$} \\
\hline & $8^{-}$ & $123^{*}$ & & & & \\
\hline & $9^{-}$ & $353^{*}$ & 821 & & & \\
\hline & $10^{-}$ & $647^{*}$ & 1025 & & & \\
\hline & $11^{-}$ & $980^{*}$ & 1332 & & & \\
\hline & $12^{-}$ & $1343^{*}$ & 1702 & & & \\
\hline & $13^{-}$ & $1733^{*}$ & 2111 & & & \\
\hline & $14^{-}$ & $2149^{*}$ & 2550 & & & \\
\hline & $15^{-}$ & 2588 & & & & \\
\hline \multirow[t]{9}{*}{${ }_{53}^{123} \mathrm{I}_{70}$} & $10.5^{+}$ & & 2876 & & & {$[87]$} \\
\hline & $11.5^{+}$ & 3083 & 3200 & $1.0\left(\begin{array}{l}+0.3 \\
-0.3\end{array}\right)$ & $2.55\left(\begin{array}{l}+0.45 \\
-0.45\end{array}\right)$ & \\
\hline & $12.5^{+}$ & $3324^{*}$ & 3490 & $1.1\left(\begin{array}{l}+0.4 \\
-0.4\end{array}\right)$ & $1.75\left(\begin{array}{l}+0.35 \\
-0.35\end{array}\right)$ & \\
\hline & $13.5^{+}$ & 3716 & 3903 & $0.9\left(\begin{array}{l}+0.5 \\
-0.5\end{array}\right)$ & $1.80\left({ }_{-0.50}^{+0.50}\right)$ & \\
\hline & $14.5^{+}$ & $4055^{*}$ & 4250 & & $1.53\left(\begin{array}{c}+0.60 \\
-0.60\end{array}\right)$ & \\
\hline & $15.5^{+}$ & 4542 & 4699 & & & \\
\hline & $16.5^{+}$ & $4901^{*}$ & & & & \\
\hline & $18.5^{+}$ & $5819^{*}$ & & & & \\
\hline & $20.5^{+}$ & $6863^{*}$ & & & & \\
\hline \multirow[t]{4}{*}{${ }_{53}^{126} \mathrm{I}_{73}$} & $8^{-}$ & $410.7^{*}$ & & & & {$[88]$} \\
\hline & $9^{-}$ & $735.1^{*}$ & & & & \\
\hline & $10^{-}$ & $1129.7^{*}$ & & $1.44\left(\begin{array}{c}+0.03 \\
-0.03\end{array}\right)$ & & \\
\hline & $11^{-}$ & $1468.4^{*}$ & 2213.7 & $1.83\left(\begin{array}{l}+0.04 \\
-0.04\end{array}\right)$ & & \\
\hline
\end{tabular}


Table 1 (continued)

\begin{tabular}{|c|c|c|c|c|c|c|}
\hline \multirow{2}{*}{ Nuclei } & \multirow{2}{*}{$I^{\pi}(\hbar)$} & \multicolumn{2}{|c|}{$E(\mathrm{keV})$} & \multicolumn{2}{|c|}{$B(M 1) / B(E 2)\left(\mu_{N}^{2} / \mathrm{e}^{2} \mathrm{~b}^{2}\right)$} & \multirow{2}{*}{ References } \\
\hline & & yrast & side & yrast & side & \\
\hline & $12^{-}$ & $1893.7^{*}$ & 2432.7 & $1.53\left(\begin{array}{l}+0.05 \\
-0.05\end{array}\right)$ & & \\
\hline & $13^{-}$ & $2322.1^{*}$ & 2707.6 & $0.22\left({ }_{-0.01}^{+0.01}\right)$ & $3.73\left(\begin{array}{l}+0.15 \\
-0.15\end{array}\right)$ & \\
\hline & $14^{-}$ & $2759.1^{*}$ & 2959.4 & $3.02\left(\begin{array}{c}+0.16 \\
-0.16\end{array}\right)$ & $5.49\left(\begin{array}{c}+0.46 \\
-0.46\end{array}\right)$ & \\
\hline & $15^{-}$ & 3290.4 & 3358.6 & $0.32\left(\begin{array}{c}+0.03 \\
-0.03\end{array}\right)$ & $9.47\left({ }_{-0.86}^{+0.86}\right)$ & \\
\hline & $16^{-}$ & 3674.5 & 3745.9 & $4.95\left({ }_{-0.42}^{+0.42}\right)$ & $4.00\left({ }_{-0.50}^{+0.50}\right)$ & \\
\hline & $17^{-}$ & 4184.1 & 4235.6 & $<1.84\left(\begin{array}{l}+0.09 \\
-0.09\end{array}\right)$ & $3.00\left(\begin{array}{c}+0.38 \\
-0.38\end{array}\right)$ & \\
\hline & $18^{-}$ & 4534.1 & 4649.9 & $1.39\left(\begin{array}{c}+0.14 \\
-0.14\end{array}\right)$ & & \\
\hline & $19^{-}$ & 5143.0 & & $<5.56\left({ }_{-0.62}^{+0.62}\right)$ & & \\
\hline & $20^{-}$ & 5528.9 & & $\sim 1.48\left(\begin{array}{l}+0.18 \\
-0.18\end{array}\right)$ & & \\
\hline & $21^{-}$ & 6231.5 & & $\sim 0.44\left({ }_{-0.06}^{+0.06}\right)$ & & \\
\hline & $22^{-}$ & 6659.7 & & & & \\
\hline \multirow[t]{12}{*}{${ }_{55}^{122} \mathrm{Cs}_{67}$} & $10^{+}$ & $368.4^{*}$ & & & & {$[89]$} \\
\hline & $11^{+}$ & $674.4^{*}$ & 915.4 & & & \\
\hline & $12^{+}$ & $840.8^{*}$ & 1221.5 & & & \\
\hline & $13^{+}$ & $1233.2^{*}$ & 1492.2 & & & \\
\hline & $14^{+}$ & $1499.9^{*}$ & 1911.7 & & & \\
\hline & $15^{+}$ & $1937.3^{*}$ & 2227.3 & & & \\
\hline & $16^{+}$ & $2313.8^{*}$ & 2757.2 & & & \\
\hline & $17^{+}$ & $2769.1^{*}$ & 3093.2 & & & \\
\hline & $18^{+}$ & $3250.8^{*}$ & & & & \\
\hline & $19^{+}$ & $3706.8^{*}$ & 4048.0 & & & \\
\hline & $20^{+}$ & $4284.7^{*}$ & & & & \\
\hline & $21^{+}$ & $4731.5^{*}$ & & & & \\
\hline \multirow[t]{11}{*}{${ }_{55}^{124} \mathrm{Cs}_{69}$} & $8^{+}$ & $561.9^{*}$ & & & & {$[90]$} \\
\hline & $9^{+}$ & $619.9^{*}$ & & & & \\
\hline & $10^{+}$ & $743.9^{*}$ & & & & \\
\hline & $11^{+}$ & $1055.9^{*}$ & 1260.2 & & & \\
\hline & $12^{+}$ & $1275.9^{*}$ & 1631.2 & & & \\
\hline & $13^{+}$ & $1673.9^{*}$ & 1893.2 & & & \\
\hline & $14^{+}$ & $1989.9^{*}$ & 2265.2 & $0.86\left(\begin{array}{c}+0.24 \\
-0.31\end{array}\right)$ & $1.37\left(\begin{array}{c}+0.60 \\
-0.63\end{array}\right)$ & \\
\hline & $15^{+}$ & $2446.9^{*}$ & 2666.2 & $3.40\left({ }_{-1.53}^{+1.11}\right)$ & $2.69\left(\begin{array}{c}+0.98 \\
-0.87\end{array}\right)$ & \\
\hline & $16^{+}$ & $2858.9^{*}$ & 3090.2 & $0.22\left(\begin{array}{c}+0.06 \\
-0.05\end{array}\right)$ & $0.42\left(\begin{array}{c}+0.16 \\
-0.16\end{array}\right)$ & \\
\hline & $17^{+}$ & $3344.9^{*}$ & 3574.2 & $2.95\left(\begin{array}{l}+1.15 \\
-0.75\end{array}\right)$ & $1.62\left(\begin{array}{c}+0.73 \\
-0.60\end{array}\right)$ & \\
\hline & $18^{+}$ & $3832.9^{*}$ & & $0.28\left(\begin{array}{l}+0.06 \\
-0.06\end{array}\right)$ & & \\
\hline
\end{tabular}


Table 1 (continued)

\begin{tabular}{|c|c|c|c|c|c|c|}
\hline \multirow{2}{*}{ Nuclei } & \multirow{2}{*}{$I^{\pi}(\hbar)$} & \multicolumn{2}{|c|}{$E(\mathrm{keV})$} & \multicolumn{2}{|c|}{$B(M 1) / B(E 2)\left(\mu_{N}^{2} / \mathrm{e}^{2} \mathrm{~b}^{2}\right)$} & \multirow{2}{*}{ References } \\
\hline & & yrast & side & yrast & side & \\
\hline & $19^{+}$ & $4342.9^{*}$ & & $3.02\left(\begin{array}{l}+0.68 \\
-0.82\end{array}\right)$ & & \\
\hline & $20^{+}$ & $4906.9^{*}$ & & $0.28\left(\begin{array}{l}+0.07 \\
-0.07\end{array}\right)$ & & \\
\hline & $21^{+}$ & $5424.9^{*}$ & & $2.44\left(\begin{array}{l}+1.19 \\
-1.14\end{array}\right)$ & & \\
\hline & $22^{+}$ & $6086.9^{*}$ & & $0.32\left({ }_{-0.06}^{+0.06}\right)$ & & \\
\hline & $23^{+}$ & 6550.9 & & $2.44\left(\begin{array}{c}+0.68 \\
-0.68\end{array}\right)$ & & \\
\hline & $24^{+}$ & 7357.9 & & & & \\
\hline & $25^{+}$ & 7649.9 & & & & \\
\hline \multirow[t]{13}{*}{${ }_{55}^{126} \mathrm{Cs}_{71}$} & $9^{+}$ & $0^{*}$ & & & & {$[91,127,128]$} \\
\hline & $10^{+}$ & $140^{*}$ & & & & \\
\hline & $11^{+}$ & $477^{*}$ & 637 & & & \\
\hline & $12^{+}$ & $732^{*}$ & 999 & & & \\
\hline & $13^{+}$ & $1128^{*}$ & 1326 & & & \\
\hline & $14^{+}$ & $1471^{*}$ & 1671 & $0.64\left(\begin{array}{l}+0.44 \\
-0.12\end{array}\right)$ & $1.26\left(\begin{array}{c}+0.64 \\
-0.26\end{array}\right)$ & \\
\hline & $15^{+}$ & $1935^{*}$ & 2097 & $4.43\left(\begin{array}{l}+3.71 \\
-1.04\end{array}\right)$ & $3.04\left(\begin{array}{l}+2.75 \\
-0.85\end{array}\right)$ & \\
\hline & $16^{+}$ & $2350^{*}$ & 2572 & $0.26\left(\begin{array}{l}+0.10 \\
-0.04\end{array}\right)$ & $1.47\left({ }_{-0.23}^{+0.90}\right)$ & \\
\hline & $17^{+}$ & $2846^{*}$ & 3033 & $2.95\left({ }_{-0.62}^{+1.96}\right)$ & $0.92\left(\begin{array}{l}+0.58 \\
-0.19\end{array}\right)$ & \\
\hline & $18^{+}$ & $3311^{*}$ & & $0.48\left(\begin{array}{l}+0.16 \\
-0.07\end{array}\right)$ & & \\
\hline & $19^{+}$ & $3839^{*}$ & 4040 & $3.63\left(\begin{array}{l}+3.50 \\
-1.05\end{array}\right)$ & & \\
\hline & $20^{+}$ & $4357^{*}$ & & $0.83\left({ }_{-0.11}^{+0.40}\right)$ & & \\
\hline & $21^{+}$ & $4911^{*}$ & & $2.65\left(\begin{array}{l}+3.75 \\
-1.21\end{array}\right)$ & & \\
\hline \multirow[t]{12}{*}{${ }_{55}^{128} \mathrm{Cs}_{73}$} & $9^{+}$ & $0^{*}$ & & & & {$[24,92,96]$} \\
\hline & $10^{+}$ & $143^{*}$ & & & & \\
\hline & $11^{+}$ & $492^{*}$ & 652 & & & \\
\hline & $12^{+}$ & $765^{*}$ & 1024 & 2.2 & & \\
\hline & $13^{+}$ & $1173^{*}$ & 1387 & 8.3 & 1.2 & \\
\hline & $14^{+}$ & $1544^{*}$ & 1744 & 0.8 & 6.9 & \\
\hline & $15^{+}$ & $2009^{*}$ & 2188 & 3.7 & 4.9 & \\
\hline & $16^{+}$ & $2419^{*}$ & 2647 & & & \\
\hline & $17^{+}$ & $2913^{*}$ & 3100 & 2.6 & 2.2 & \\
\hline & $18^{+}$ & $3349^{*}$ & & & & \\
\hline & $19^{+}$ & $3864^{*}$ & & & & \\
\hline & $20^{+}$ & $4336^{*}$ & & & & \\
\hline \multirow[t]{2}{*}{${ }_{55}^{130} \mathrm{Cs}_{75}$} & $10^{+}$ & $959.7^{*}$ & & & & {$[24,86,93,129-131]$} \\
\hline & $11^{+}$ & $1312.7^{*}$ & 1506.4 & & & \\
\hline
\end{tabular}


Table 1 (continued)

\begin{tabular}{|c|c|c|c|c|c|c|}
\hline \multirow{2}{*}{ Nuclei } & \multirow{2}{*}{$I^{\pi}(\hbar)$} & \multicolumn{2}{|c|}{$E(\mathrm{keV})$} & \multicolumn{2}{|c|}{$B(M 1) / B(E 2)\left(\mu_{N}^{2} / \mathrm{e}^{2} \mathrm{~b}^{2}\right)$} & \multirow{2}{*}{ References } \\
\hline & & yrast & side & yrast & side & \\
\hline & $12^{+}$ & $1602.4^{*}$ & 1907.7 & $4.12\left(\begin{array}{l}+1.77 \\
-1.39\end{array}\right)$ & & \\
\hline & $13^{+}$ & $2019.0^{*}$ & 2280.7 & $6.75\left(\begin{array}{l}+2.18 \\
-2.26\end{array}\right)$ & $2.13\left({ }_{-1.02}^{+1.41}\right)$ & \\
\hline & $14^{+}$ & $2445.1^{*}$ & 2632.0 & $1.71\left(\begin{array}{c}+0.94 \\
-0.70\end{array}\right)$ & $2.50\left(\begin{array}{l}+0.67 \\
-1.35\end{array}\right)$ & \\
\hline & $15^{+}$ & $2913.5^{*}$ & 3085.0 & $4.84\left(\begin{array}{l}+3.99 \\
-2.93\end{array}\right)$ & $7.84\left(\begin{array}{c}+7.70 \\
-4.91\end{array}\right)$ & \\
\hline & $16^{+}$ & $3379.1^{*}$ & 3563.4 & $1.00\left(\begin{array}{c}+0.24 \\
-0.24\end{array}\right)$ & & \\
\hline & $17^{+}$ & $3871.0^{*}$ & 4038.4 & $5.66\left(\begin{array}{c}+4.53 \\
-3.24\end{array}\right)$ & 4.04 & \\
\hline & $18^{+}$ & 4320.8 & & 3.25 & & \\
\hline & $19^{+}$ & 4859.0 & 5105.1 & & & \\
\hline & $20^{+}$ & 5338.4 & & & & \\
\hline & $21^{+}$ & 5899.3 & & & & \\
\hline & $22^{+}$ & 6450.3 & & & & \\
\hline & $23^{+}$ & 6930.6 & & & & \\
\hline & $24^{+}$ & 7487.5 & & & & \\
\hline & $26^{+}$ & 8622.0 & & & & \\
\hline \multirow[t]{11}{*}{${ }_{55}^{132} \mathrm{Cs}_{77}$} & $9^{+}$ & 1131 & & & & {$[24,94,132]$} \\
\hline & $10^{+}$ & 1282 & 1729 & & & \\
\hline & $11^{+}$ & 1683 & 1891 & & & \\
\hline & $12^{+}$ & 1982 & 2202 & $1.3\left(\begin{array}{l}+0.2 \\
-0.2\end{array}\right)$ & & \\
\hline & $13^{+}$ & 2410 & 2515 & $5.9\left(\begin{array}{c}+1.0 \\
-1.0\end{array}\right)$ & & \\
\hline & $14^{+}$ & 2865 & 2894 & $1.4\left(\begin{array}{l}+0.3 \\
-0.3\end{array}\right)$ & & \\
\hline & $15^{+}$ & 3311 & & $6.1\left({ }_{-0.7}^{+0.7}\right)$ & & \\
\hline & $16^{+}$ & 3789 & & $2.2\left(\begin{array}{l}+0.6 \\
-0.6\end{array}\right)$ & & \\
\hline & $17^{+}$ & 4241 & & $3.1\left(\begin{array}{l}+0.6 \\
-0.6\end{array}\right)$ & & \\
\hline & $18^{+}$ & 4665 & & $4.0\left(\begin{array}{l}+1.4 \\
-1.4\end{array}\right)$ & & \\
\hline & $19^{+}$ & 5074 & & & & \\
\hline \multirow[t]{9}{*}{${ }_{57}^{128} \mathrm{La}_{71}$} & $8^{+}$ & $0.0^{*}$ & & & & {$[95]$} \\
\hline & $9^{+}$ & $104.0^{*}$ & & & & \\
\hline & $10^{+}$ & $242.5^{*}$ & & $1.87487\left(\begin{array}{l}+0.37497 \\
-0.37497\end{array}\right)$ & & \\
\hline & $11^{+}$ & $477.7^{*}$ & 808.2 & $2.27708\left(\begin{array}{l}+0.45542 \\
-0.45542\end{array}\right)$ & & \\
\hline & $12^{+}$ & $700.0^{*}$ & 1078.7 & $2.03719\left(\begin{array}{l}+0.40744 \\
-0.40744\end{array}\right)$ & & \\
\hline & $13^{+}$ & $1035.0^{*}$ & 1465.8 & $2.39101\left(\begin{array}{l}+0.47820 \\
-0.47820\end{array}\right)$ & $1.38023\left({ }_{-0.82814}^{+0.82814}\right)$ & \\
\hline & $14^{+}$ & $1334.0^{*}$ & 1778.1 & $1.76221\left(\begin{array}{l}+0.35244 \\
-0.35244\end{array}\right)$ & $1.64005\left({ }_{-0.98403}^{+0.98403}\right)$ & \\
\hline & $15^{+}$ & $1752.6^{*}$ & 2209.9 & $1.97941\left(\begin{array}{l}+0.39588 \\
-0.39588\end{array}\right)$ & $1.50467\left({ }_{-0.90280}^{+0.90280}\right)$ & \\
\hline & $16^{+}$ & $2121.0^{*}$ & 2580.3 & $1.60661\left(\begin{array}{l}+0.32132 \\
-0.32132\end{array}\right)$ & $2.16842\left({ }_{-1.30105}^{+1.30105}\right)$ & \\
\hline
\end{tabular}


Table 1 (continued)

\begin{tabular}{|c|c|c|c|c|c|c|}
\hline \multirow{2}{*}{ Nuclei } & \multirow{2}{*}{$I^{\pi}(\hbar)$} & \multicolumn{2}{|c|}{$E(\mathrm{keV})$} & \multicolumn{2}{|c|}{$B(M 1) / B(E 2)\left(\mu_{N}^{2} / \mathrm{e}^{2} \mathrm{~b}^{2}\right)$} & \multirow{2}{*}{ References } \\
\hline & & yrast & side & yrast & side & \\
\hline & $17^{+}$ & $2611.4^{*}$ & 3129.4 & $1.25884\left(\begin{array}{l}+0.50354 \\
-0.50354\end{array}\right)$ & & \\
\hline & $18^{+}$ & $3044.2^{*}$ & 3551.3 & $1.23285\left(\begin{array}{l}+0.49314 \\
-0.49314\end{array}\right)$ & & \\
\hline & $19^{+}$ & $3592.9^{*}$ & 4144.4 & $1.32745\left(\begin{array}{l}+0.51771 \\
-0.51771\end{array}\right)$ & & \\
\hline & $20^{+}$ & $4090.1^{*}$ & 4593.3 & & & \\
\hline & $21^{+}$ & $4679.9^{*}$ & & & & \\
\hline & $22^{+}$ & 5243.4 & & & & \\
\hline & $23^{+}$ & 5855.3 & & & & \\
\hline & $24^{+}$ & 6486.4 & & & & \\
\hline \multirow[t]{11}{*}{${ }_{57}^{130} \mathrm{La}_{73}$} & 9 & 0 & & & & {$[96]$} \\
\hline & 10 & 138 & & & & \\
\hline & 11 & 417 & 693 & & & \\
\hline & 12 & 663 & 1050 & & & \\
\hline & 13 & 1037 & 1393 & & & \\
\hline & 14 & $1363^{*}$ & 1778 & & & \\
\hline & 15 & $1809^{*}$ & 2204 & & & \\
\hline & 16 & $2202^{*}$ & 2576 & & & \\
\hline & 17 & $2712^{*}$ & & & & \\
\hline & 18 & $3157^{*}$ & & & & \\
\hline & 20 & $4208^{*}$ & & & & \\
\hline \multirow[t]{12}{*}{${ }_{57}^{132} \mathrm{La}_{75}$} & $10^{+}$ & $936.4^{*}$ & & & & {$[23,86,92,97,129]$} \\
\hline & $11^{+}$ & $1229.4^{*}$ & 1558.4 & & & \\
\hline & $12^{+}$ & $1523.4^{*}$ & 1919.4 & $7.8\left({ }_{-0.4}^{+0.4}\right)$ & & \\
\hline & $13^{+}$ & $1915.4^{*}$ & 2299.4 & $7.5\left(\begin{array}{l}+0.4 \\
-0.4\end{array}\right)$ & & \\
\hline & $14^{+}$ & $2300.4^{*}$ & 2703.4 & $5.7\left(\begin{array}{l}+0.1 \\
-0.1\end{array}\right)$ & & \\
\hline & $15^{+}$ & $2753.4^{*}$ & 3130.4 & $5.7\left(\begin{array}{l}+0.2 \\
-0.2\end{array}\right)$ & & \\
\hline & $16^{+}$ & $3205.4^{*}$ & & $5.2\left(\begin{array}{l}+0.2 \\
-0.2\end{array}\right)$ & & \\
\hline & $17^{+}$ & $3712.4^{*}$ & & $6.8\left({ }_{-0.4}^{+0.4}\right)$ & & \\
\hline & $18^{+}$ & $4199.4^{*}$ & & $4.9\left(\begin{array}{l}+0.3 \\
-0.3\end{array}\right)$ & & \\
\hline & $19^{+}$ & $4758.4^{*}$ & & $3.4\left(\begin{array}{l}+0.4 \\
-0.4\end{array}\right)$ & & \\
\hline & $20^{+}$ & $5218.4^{*}$ & & & & \\
\hline & $22^{+}$ & 6337.4 & & & & \\
\hline \multirow[t]{3}{*}{${ }_{57}^{133} \mathrm{La}_{76}$} & $7.5^{+}$ & 2290 & & & & {$[98]$} \\
\hline & $8.5^{+}$ & 2367 & & & & \\
\hline & $9.5^{+}$ & 2502 & 2726 & $5.7\left(\begin{array}{l}+3.3 \\
-3.3\end{array}\right)$ & & \\
\hline
\end{tabular}


Table 1 (continued)

\begin{tabular}{|c|c|c|c|c|c|c|}
\hline \multirow{2}{*}{ Nuclei } & \multirow{2}{*}{$I^{\pi}(\hbar)$} & \multicolumn{2}{|c|}{$E(\mathrm{keV})$} & \multicolumn{2}{|c|}{$B(M 1) / B(E 2)\left(\mu_{N}^{2} / \mathrm{e}^{2} \mathrm{~b}^{2}\right)$} & \multirow{2}{*}{ References } \\
\hline & & yrast & side & yrast & side & \\
\hline & $10.5^{+}$ & 2681 & 2893 & $20.0\left(\begin{array}{c}+10.0 \\
-10.0\end{array}\right)$ & & \\
\hline & $11.5^{+}$ & 2927 & 3110 & $6.7\left(\begin{array}{l}+1.7 \\
-1.7\end{array}\right)$ & $4.2543\left(\begin{array}{l}+1.2181 \\
-1.2181\end{array}\right)$ & \\
\hline & $12.5^{+}$ & 3258 & 3382 & $4.7\left(\begin{array}{l}+1.1 \\
-1.1\end{array}\right)$ & $3.78366\left({ }_{-2.32055}^{+2.32055}\right)$ & \\
\hline & $13.5^{+}$ & 3614 & 3778 & $7.3\left({ }_{-0.7}^{+0.7}\right)$ & $4.7\left(\begin{array}{l}+2.2 \\
-2.2\end{array}\right)$ & \\
\hline & $14.5^{+}$ & 4030 & 4134 & $18.4\left(\begin{array}{l}+5.0 \\
-5.0\end{array}\right)$ & $10.5\left(\begin{array}{l}+4.0 \\
-4.0\end{array}\right)$ & \\
\hline & $15.5^{+}$ & 4475 & & $5.8\left(\begin{array}{l}+1.0 \\
-1.0\end{array}\right)$ & & \\
\hline & $16.5^{+}$ & 4906 & & $3.0\left(\begin{array}{l}+2.0 \\
-2.0\end{array}\right)$ & & \\
\hline & $17.5^{+}$ & 5352 & & $2.0\left(\begin{array}{c}+1.2 \\
-1.2\end{array}\right)$ & & \\
\hline & $19.5^{+}$ & 6283 & & & & \\
\hline \multirow[t]{15}{*}{${ }_{57}^{134} \mathrm{La}_{77}$} & $10^{+}$ & $813^{*}$ & & & & {$[99]$} \\
\hline & $11^{+}$ & $1195^{*}$ & 1412 & & & \\
\hline & $12^{+}$ & $1533^{*}$ & 1710 & $3.6\left(\begin{array}{l}+0.2 \\
-0.2\end{array}\right)$ & & \\
\hline & $13^{+}$ & $1969^{*}$ & 2051 & $12.6\left({ }_{-0.6}^{+0.6}\right)$ & & \\
\hline & $14^{+}$ & 2404 & 2418 & $3.4\left(\begin{array}{l}+0.2 \\
-0.2\end{array}\right)$ & & \\
\hline & $15^{+}$ & 2849 & & $10.3\left({ }_{-0.6}^{+0.6}\right)$ & & \\
\hline & $16^{+}$ & 3284 & & $10.0\left(\begin{array}{l}+0.8 \\
-0.8\end{array}\right)$ & & \\
\hline & $17^{+}$ & $3775^{*}$ & & $10.1\left(\begin{array}{l}+0.7 \\
-0.7\end{array}\right)$ & & \\
\hline & $18^{+}$ & $4120^{*}$ & & $1.1\left({ }_{-0.1}^{+0.1}\right)$ & & \\
\hline & $19^{+}$ & $4698^{*}$ & & $8.0\left(\begin{array}{l}+0.7 \\
-0.7\end{array}\right)$ & & \\
\hline & $20^{+}$ & $5079^{*}$ & & $1.0\left(\begin{array}{l}+0.2 \\
-0.2\end{array}\right)$ & & \\
\hline & $21^{+}$ & 5714 & & $4.6\left({ }_{-0.3}^{+0.3}\right)$ & & \\
\hline & $22^{+}$ & $6137^{*}$ & & & & \\
\hline & $23^{+}$ & $6741^{*}$ & & & & \\
\hline & $24^{+}$ & $7179^{*}$ & & & & \\
\hline \multirow[t]{10}{*}{${ }_{58}^{133} \mathrm{Ce}_{75}$} & $8.5^{+}$ & 2249 & & & & {$[53]$} \\
\hline & $9.5^{+}$ & 2379 & 2465 & & & \\
\hline & $10.5^{+}$ & 2585 & 2706 & & & \\
\hline & $11.5^{+}$ & 2809 & 2922 & $9.711\left({ }_{-0.153}^{+0.153}\right)$ & & \\
\hline & $12.5^{+}$ & 3093 & 3199 & $9.522\left(\begin{array}{l}+0.134 \\
-0.134\end{array}\right)$ & $9.393\left(\begin{array}{l}+0.102 \\
-0.102\end{array}\right)$ & \\
\hline & $13.5^{+}$ & 3399 & 3534 & $9.621\left(\begin{array}{l}+0.142 \\
-0.142\end{array}\right)$ & $9.834\left(\begin{array}{l}+0.191 \\
-0.191\end{array}\right)$ & \\
\hline & $14.5^{+}$ & 3745 & 3933 & $9.471\left({ }_{-0.136}^{+0.136}\right)$ & $9.337\left({ }_{-0.143}^{+0.143}\right)$ & \\
\hline & $15.5^{+}$ & 4177 & 4334 & $9.591\left(\begin{array}{l}+0.127 \\
-0.127\end{array}\right)$ & $9.459\left(\begin{array}{l}+0.154 \\
-0.154\end{array}\right)$ & \\
\hline & $16.5^{+}$ & 4623 & 4775 & $9.428\left(\left(_{-0.143}^{+0.143}\right)\right.$ & $9.435\left(\begin{array}{l}+0.152 \\
-0.152\end{array}\right)$ & \\
\hline & $11.5^{-}$ & 3198 & & & & {$[53]$} \\
\hline
\end{tabular}


Table 1 (continued)

\begin{tabular}{|c|c|c|c|c|c|c|}
\hline \multirow{2}{*}{ Nuclei } & \multirow{2}{*}{$I^{\pi}(\hbar)$} & \multicolumn{2}{|c|}{$E(\mathrm{keV})$} & \multicolumn{2}{|c|}{$B(M 1) / B(E 2)\left(\mu_{N}^{2} / \mathrm{e}^{2} \mathrm{~b}^{2}\right)$} & \multirow{2}{*}{ References } \\
\hline & & yrast & side & yrast & side & \\
\hline & $12.5^{-}$ & 3338 & & & & \\
\hline & $13.5^{-}$ & 3493 & & & & \\
\hline & $14.5^{-}$ & 3733 & 4162 & & & \\
\hline & $15.5^{-}$ & 4028 & 4445 & $11.8286\left({ }_{-0.1243}^{+0.1243}\right)$ & & \\
\hline & $16.5^{-}$ & 4370 & 4808 & $11.2305\left({ }_{-0.2352}^{+0.2352}\right)$ & & \\
\hline & $17.5^{-}$ & 4761 & 5175 & $11.6328\left({ }_{-0.1235}^{+0.1235}\right)$ & $10.8364\left(\begin{array}{l}+0.1235 \\
-0.1235\end{array}\right)$ & \\
\hline & $18.5^{-}$ & 5177 & 5617 & $11.0246\left(\begin{array}{l}+0.1253 \\
-0.1253\end{array}\right)$ & $10.4323\left(\begin{array}{l}+0.1253 \\
-0.1253\end{array}\right)$ & \\
\hline & $19.5^{-}$ & 5632 & 6040 & $10.9486\left({ }_{-0.2104}^{+0.2104}\right)$ & $10.5896\left({ }_{-0.2104}^{+0.2104}\right)$ & \\
\hline & $20.5^{-}$ & 6112 & 6508 & $10.9207\left(\begin{array}{l}+0.1345 \\
-0.1345\end{array}\right)$ & $10.3983\left(\begin{array}{l}+0.1345 \\
-0.1345\end{array}\right)$ & \\
\hline & $21.5^{-}$ & 6624 & 6999 & $10.6351\left({ }_{-0.1423}^{+0.1423}\right)$ & $10.4571\left({ }_{-0.1423}^{+0.1423}\right)$ & \\
\hline & $22.5^{-}$ & 7167 & 7557 & $10.7351\left(\begin{array}{l}+0.2134 \\
-0.2134\end{array}\right)$ & $10.2723\left(\begin{array}{l}+0.2134 \\
-0.2134\end{array}\right)$ & \\
\hline & $23.5^{-}$ & 7739 & & $10.4997\left({ }_{-0.2365}^{+0.2365}\right)$ & & \\
\hline & $24.5^{-}$ & 8339 & & & & \\
\hline \multirow[t]{11}{*}{${ }_{59}^{132} \operatorname{Pr}_{73}$} & 9 & 0 & & & & {$[96,133]$} \\
\hline & 10 & $131^{*}$ & & $3.3\left(\begin{array}{l}+0.5 \\
-0.5\end{array}\right)$ & & \\
\hline & 11 & $397^{*}$ & 727 & $2.3\left(\begin{array}{l}+0.3 \\
-0.3\end{array}\right)$ & & \\
\hline & 12 & $638^{*}$ & 1033 & $2.5\left(\begin{array}{l}+0.3 \\
-0.3\end{array}\right)$ & & \\
\hline & 13 & $1021^{*}$ & 1458 & $2.6\left(\begin{array}{l}+0.3 \\
-0.3\end{array}\right)$ & & \\
\hline & 14 & $1342^{*}$ & 1827 & $1.7\left(\begin{array}{l}+0.2 \\
-0.2\end{array}\right)$ & & \\
\hline & 15 & $1795^{*}$ & & $2.4\left({ }_{-0.1}^{+0.1}\right)$ & & \\
\hline & 16 & $2190^{*}$ & & $1.6\left(\begin{array}{l}+0.5 \\
-0.5\end{array}\right)$ & & \\
\hline & 17 & 2675 & & $2.0\left(\begin{array}{l}+1.0 \\
-1.0\end{array}\right)$ & & \\
\hline & 18 & $3133^{*}$ & & $1.3\left(\begin{array}{l}+0.3 \\
-0.3\end{array}\right)$ & & \\
\hline & 19 & 3608 & & $1.6\left(\begin{array}{l}+0.5 \\
-0.5\end{array}\right)$ & & \\
\hline \multirow[t]{10}{*}{${ }_{59}^{134} \operatorname{Pr}_{75}$} & $9^{+}$ & 559 & 797 & & & {$[26,86,100,129,134-137]$} \\
\hline & $10^{+}$ & 764 & 931 & & & \\
\hline & $11^{+}$ & 930 & 1142 & & & \\
\hline & $12^{+}$ & 1213 & 1409 & & & \\
\hline & $13^{+}$ & $1537^{*}$ & 1659 & $35.22\left({ }_{-0.11}^{+0.11}\right)$ & & \\
\hline & $14^{+}$ & 1874 & 2017 & $150.52\left({ }_{-0.43}^{+0.43}\right)$ & & \\
\hline & $15^{+}$ & $2243^{*}$ & 2456 & $37.11\left({ }_{-0.12}^{+0.12}\right)$ & $7.42\left(\begin{array}{c}+0.02 \\
-0.02\end{array}\right)$ & \\
\hline & $16^{+}$ & 2643 & 2901 & $7.89\left(\begin{array}{l}+0.02 \\
-0.02\end{array}\right)$ & $7.93\left(\begin{array}{l}+0.02 \\
-0.02\end{array}\right)$ & \\
\hline & $17^{+}$ & 3115 & 3347 & $8.59\left(\begin{array}{l}+0.02 \\
-0.02\end{array}\right)$ & $10.97\left({ }_{-0.04}^{+0.04}\right)$ & \\
\hline & $18^{+}$ & 3582 & 3858 & $4.20\left(\begin{array}{l}+0.01 \\
-0.01\end{array}\right)$ & $9.78\left(\begin{array}{l}+0.03 \\
-0.03\end{array}\right)$ & \\
\hline
\end{tabular}


Table 1 (continued)

\begin{tabular}{|c|c|c|c|c|c|c|}
\hline \multirow{2}{*}{ Nuclei } & \multirow{2}{*}{$I^{\pi}(\hbar)$} & \multicolumn{2}{|c|}{$E(\mathrm{keV})$} & \multicolumn{2}{|c|}{$B(M 1) / B(E 2)\left(\mu_{N}^{2} / \mathrm{e}^{2} \mathrm{~b}^{2}\right)$} & \multirow{2}{*}{ References } \\
\hline & & yrast & side & yrast & side & \\
\hline & $19^{+}$ & 4172 & 4338 & $3.08\left(\begin{array}{l}+0.01 \\
-0.01\end{array}\right)$ & $9.04\left(\begin{array}{l}+0.04 \\
-0.04\end{array}\right)$ & \\
\hline & $20^{+}$ & 4648 & & $2.12\left(\begin{array}{l}+0.01 \\
-0.01\end{array}\right)$ & & \\
\hline & $21^{+}$ & 5343 & & $4.57\left(\begin{array}{l}+0.01 \\
-0.01\end{array}\right)$ & & \\
\hline & $22^{+}$ & 5839 & & & & \\
\hline & $23^{+}$ & 6534 & & & & \\
\hline \multirow[t]{12}{*}{${ }_{60}^{135} \mathrm{Nd}_{75}$} & $11.5^{-}$ & 2819.4 & & & & {$[38,138]$} \\
\hline & $12.5^{-}$ & 2940.4 & & & & \\
\hline & $13.5^{-}$ & 3110.5 & 3607.4 & & & \\
\hline & $14.5^{-}$ & $3358.2^{*}$ & 3780.4 & $10.00\left(\begin{array}{l}+2.26 \\
-2.26\end{array}\right)$ & & \\
\hline & $15.5^{-}$ & $3649.5^{*}$ & 4006.4 & $7.81\left(\begin{array}{l}+1.35 \\
-1.35\end{array}\right)$ & $9.64\left(\begin{array}{l}+1.30 \\
-1.30\end{array}\right)$ & \\
\hline & $16.5^{-}$ & $4007.7^{*}$ & 4288.4 & $6.88\left(\begin{array}{l}+2.30 \\
-2.30\end{array}\right)$ & $7.50\left(\begin{array}{l}+2.11 \\
-2.11\end{array}\right)$ & \\
\hline & $17.5^{-}$ & $4413.7^{*}$ & 4597.4 & $7.50\left(\begin{array}{l}+1.33 \\
-1.33\end{array}\right)$ & $7.86\left({ }_{-2.61}^{+2.61}\right)$ & \\
\hline & $18.5^{-}$ & $4852.7^{*}$ & 4969.4 & $5.31\left(\begin{array}{l}+1.31 \\
-1.31\end{array}\right)$ & $5.86\left(\begin{array}{l}+2.23 \\
-2.23\end{array}\right)$ & \\
\hline & $19.5^{-}$ & $5315.7^{*}$ & 5409.4 & $16.15\left({ }_{-0.82}^{+0.82}\right)$ & $17.27\left(\begin{array}{l}+0.73 \\
-0.73\end{array}\right)$ & \\
\hline & $20.5^{-}$ & $5787.7^{*}$ & 5921.4 & $11.05\left({ }_{-0.94}^{+0.94}\right)$ & & \\
\hline & $21.5^{-}$ & $6281.7^{*}$ & & $9.52\left(\begin{array}{l}+1.13 \\
-1.13\end{array}\right)$ & & \\
\hline & $22.5^{-}$ & 6799.7 & & & & \\
\hline \multirow[t]{17}{*}{${ }_{60}^{136} \mathrm{Nd}_{76}$} & $11^{+}$ & 4445 & & & & {$[56]$} \\
\hline & $12^{+}$ & 4665 & & & & \\
\hline & $13^{+}$ & 4919 & & & & \\
\hline & $14^{+}$ & 5213 & & & & \\
\hline & $15^{+}$ & 5558 & 5635 & $4.0\left(\begin{array}{l}+1.3 \\
-1.3\end{array}\right)$ & & \\
\hline & $16^{+}$ & 5969 & 6053 & $31\left(\begin{array}{l}+17 \\
-17\end{array}\right)$ & & \\
\hline & $17^{+}$ & 6423 & 6485 & $7.0\left(\begin{array}{l}+4.8 \\
-4.8\end{array}\right)$ & & \\
\hline & $18^{+}$ & 6908 & 6989 & & & \\
\hline & $19^{+}$ & 7502 & 7468 & & & \\
\hline & $20^{+}$ & 8115 & & & & \\
\hline & $15^{+}$ & 6229 & & & & {$[56]$} \\
\hline & $16^{+}$ & 6347 & & & & \\
\hline & $17^{+}$ & 6578 & & 38 & & \\
\hline & $18^{+}$ & 6883 & & 14 & & \\
\hline & $19^{+}$ & 7292 & & 39.7 & & \\
\hline & $20^{+}$ & 7668 & & $93\left(\begin{array}{l}+28 \\
-28\end{array}\right)$ & & \\
\hline & $21^{+}$ & 8049 & 8412 & 453.2 & & \\
\hline
\end{tabular}


Table 1 (continued)

\begin{tabular}{|c|c|c|c|c|c|c|}
\hline \multirow{2}{*}{ Nuclei } & \multirow{2}{*}{$I^{\pi}(\hbar)$} & \multicolumn{2}{|c|}{$E(\mathrm{keV})$} & \multicolumn{2}{|c|}{$B(M 1) / B(E 2)\left(\mu_{N}^{2} / \mathrm{e}^{2} \mathrm{~b}^{2}\right)$} & \multirow{2}{*}{ References } \\
\hline & & yrast & side & yrast & side & \\
\hline & $22^{+}$ & 8465 & 8838 & 197.7 & & \\
\hline & $23^{+}$ & 8946 & 9247 & 115 & & \\
\hline & $24^{+}$ & 9489 & 9694 & & & \\
\hline & $25^{+}$ & 10089 & 10204 & & & \\
\hline & $26^{+}$ & 10761 & 10657 & & & \\
\hline & $27^{+}$ & & 11256 & & & \\
\hline & $13^{-}$ & 5348 & & & & {$[56]$} \\
\hline & $14^{-}$ & 5531 & & & & \\
\hline & $15^{-}$ & 5731 & & $7.37\left(\begin{array}{l}+1.72 \\
-1.83\end{array}\right)$ & & \\
\hline & $16^{-}$ & 5980 & & $3.73\left(\begin{array}{c}+1.59 \\
-1.60\end{array}\right)$ & & \\
\hline & $17^{-}$ & 6325 & & $11.00\left(\begin{array}{c}+1.49 \\
-1.50\end{array}\right)$ & & \\
\hline & $18^{-}$ & 6759 & & & & \\
\hline & $19^{-}$ & 7225 & 7270 & & & \\
\hline & $20^{-}$ & 7720 & 7721 & $54.30\left({ }_{-24.63}^{+23.20}\right)$ & & \\
\hline & $21^{-}$ & 8169 & 8214 & $41.84\left({ }_{-9.27}^{+9.15}\right)$ & & \\
\hline & $22^{-}$ & 8689 & 8751 & & & \\
\hline & $23^{-}$ & 9231 & & & & \\
\hline & $24^{-}$ & 9786 & & $8.69\left(\begin{array}{l}+5.05 \\
-5.06\end{array}\right)$ & & \\
\hline & $14^{-}$ & 5418 & & & & {$[56,139,140]$} \\
\hline & $15^{-}$ & 5648 & & & & \\
\hline & $16^{-}$ & 5957 & & $7.51\left({ }_{-1.54}^{+1.52}\right)$ & & \\
\hline & $17^{-}$ & 6314 & & $14.93\left({ }_{-1.62}^{+1.49}\right)$ & & \\
\hline & $18^{-}$ & 6703 & & $20.13\left({ }_{-4.23}^{+4.07}\right)$ & & \\
\hline & $19^{-}$ & 7139 & 7259 & $48.43\left({ }_{-8.92}^{+9.08}\right)$ & & \\
\hline & $20^{-}$ & 7566 & 7725 & $43.19\left({ }_{-11.75}^{+11.42}\right)$ & & \\
\hline & $21^{-}$ & 8011 & 8199 & $34.79\left(\begin{array}{c}+5.48 \\
-4.92\end{array}\right)$ & & \\
\hline & $22^{-}$ & 8499 & 8616 & $18.65\left({ }_{-2.20}^{+2.03}\right)$ & & \\
\hline & $23^{-}$ & 9009 & 9080 & $5.94\left(\begin{array}{l}+3.21 \\
-3.27\end{array}\right)$ & & \\
\hline & $24^{-}$ & 9559 & 9671 & $3.20\left(\begin{array}{l}+0.96 \\
-0.96\end{array}\right)$ & & \\
\hline & $25^{-}$ & 10190 & & & & \\
\hline & $15^{+}$ & 5826 & & & & {$[56]$} \\
\hline & $16^{+}$ & 6006 & & & & \\
\hline & $17^{+}$ & $6238^{*}$ & & $4.00\left({ }_{-2.00}^{+2.00}\right)$ & & \\
\hline & $18^{+}$ & 6522 & 6932 & $17.36\left(\begin{array}{l}+9.00 \\
-9.00\end{array}\right)$ & & \\
\hline
\end{tabular}


Table 1 (continued)

\begin{tabular}{|c|c|c|c|c|c|c|}
\hline \multirow{2}{*}{ Nuclei } & \multirow{2}{*}{$I^{\pi}(\hbar)$} & \multicolumn{2}{|c|}{$E(\mathrm{keV})$} & \multicolumn{2}{|c|}{$B(M 1) / B(E 2)\left(\mu_{N}^{2} / \mathrm{e}^{2} \mathrm{~b}^{2}\right)$} & \multirow{2}{*}{ References } \\
\hline & & yrast & side & yrast & side & \\
\hline & $19^{+}$ & $6867^{*}$ & 7213 & $7.57\left(\begin{array}{l}+0.97 \\
-0.97\end{array}\right)$ & & \\
\hline & $20^{+}$ & $7255^{*}$ & 7543 & $7.87\left(\begin{array}{l}+2.10 \\
-2.10\end{array}\right)$ & $3.22\left({ }_{-2.04}^{+2.00}\right)$ & \\
\hline & $21^{+}$ & $7685^{*}$ & 7927 & $5.63\left(\begin{array}{l}+0.75 \\
-0.79\end{array}\right)$ & $7.04\left(\begin{array}{l}+2.98 \\
-3.07\end{array}\right)$ & \\
\hline & $22^{+}$ & $8148^{*}$ & 8355 & $6.35\left(\begin{array}{l}+0.76 \\
-0.74\end{array}\right)$ & $7.67\left({ }_{-5.08}^{+4.70}\right)$ & \\
\hline & $23^{+}$ & $8652^{*}$ & 8828 & $6.48\left(\begin{array}{l}+1.30 \\
-1.30\end{array}\right)$ & $6.46\left(\begin{array}{l}+2.92 \\
-2.98\end{array}\right)$ & \\
\hline & $24^{+}$ & $9178^{*}$ & 9347 & $6.60\left(\begin{array}{l}+3.00 \\
-3.00\end{array}\right)$ & $5.98\left(\begin{array}{l}+4.03 \\
-0.17\end{array}\right)$ & \\
\hline & $25^{+}$ & $9745^{*}$ & 9911 & $3.70\left({ }_{-2.00}^{+2.00}\right)$ & & \\
\hline & $26^{+}$ & $10345^{*}$ & 10504 & $2.69\left(\begin{array}{c}+1.90 \\
-1.90\end{array}\right)$ & & \\
\hline & $27^{+}$ & $10967^{*}$ & 11117 & & & \\
\hline & $28^{+}$ & $11651^{*}$ & & & & \\
\hline & $29^{+}$ & $12335^{*}$ & & & & \\
\hline \multirow[t]{13}{*}{${ }_{60}^{137} \mathrm{Nd}_{77}$} & $13.5^{-}$ & 3895 & & & & {$[101]$} \\
\hline & $14.5^{-}$ & 4159 & & & & \\
\hline & $15.5^{-}$ & 4513 & 4821 & 15.1 & & \\
\hline & $16.5^{-}$ & $4909^{*}$ & 5107 & $13.7\left(\begin{array}{l}+4.9 \\
-4.9\end{array}\right)$ & & \\
\hline & $17.5^{-}$ & $5372^{*}$ & 5415 & $21.7\left(\begin{array}{l}+6.5 \\
-6.5\end{array}\right)$ & & \\
\hline & $18.5^{-}$ & 5812 & $5787^{*}$ & $39.2\left(\begin{array}{l}+15.7 \\
-15.7\end{array}\right)$ & & \\
\hline & $19.5^{-}$ & 6193 & 6262 & $20.9\left(\begin{array}{l}+6.8 \\
-6.8\end{array}\right)$ & & \\
\hline & $20.5^{-}$ & $6668^{*}$ & 6794 & $9.0\left(\begin{array}{l}+4.7 \\
-4.7\end{array}\right)$ & & \\
\hline & $21.5^{-}$ & $7099^{*}$ & 7313 & $5.8\left({ }_{-2.1}^{+2.1}\right)$ & & \\
\hline & $22.5^{-}$ & $7650^{*}$ & 7701 & $5.7\left(\begin{array}{l}+3.4 \\
-3.4\end{array}\right)$ & & \\
\hline & $23.5^{-}$ & 8347 & 8196 & $9.4\left(\begin{array}{l}+5.6 \\
-5.6\end{array}\right)$ & & \\
\hline & $24.5^{-}$ & & 8744 & & & \\
\hline & $25.5^{-}$ & & $9336^{*}$ & & & \\
\hline \multirow[t]{10}{*}{${ }_{60}^{138} \mathrm{Nd}_{78}$} & $10^{+}$ & & 4344 & & & {$[30,63]$} \\
\hline & $11^{+}$ & 4381 & 4546 & & & \\
\hline & $12^{+}$ & 4737 & 4779 & & & \\
\hline & $13^{+}$ & & 5069 & & & \\
\hline & $14^{+}$ & & 5363 & & & \\
\hline & $15^{+}$ & & 5678 & & & \\
\hline & $16^{+}$ & & 6179 & & & \\
\hline & $17^{+}$ & & 6760 & & & \\
\hline & $13^{-}$ & 5493 & & & & {$[63]$} \\
\hline & $14^{-}$ & 5577 & & & & \\
\hline
\end{tabular}


Table 1 (continued)

\begin{tabular}{|c|c|c|c|c|c|c|}
\hline \multirow{2}{*}{ Nuclei } & \multirow{2}{*}{$I^{\pi}(\hbar)$} & \multicolumn{2}{|c|}{$E(\mathrm{keV})$} & \multicolumn{2}{|c|}{$B(M 1) / B(E 2)\left(\mu_{N}^{2} / \mathrm{e}^{2} \mathrm{~b}^{2}\right)$} & \multirow{2}{*}{ References } \\
\hline & & yrast & side & yrast & side & \\
\hline & $15^{-}$ & 5770 & 6018 & 6.5 & & \\
\hline & $16^{-}$ & 6001 & 6285 & 14.1 & & \\
\hline & $17^{-}$ & 6287 & 6560 & 11.2 & & \\
\hline & $18^{-}$ & 6668 & 6909 & 31.6 & & \\
\hline & $19^{-}$ & $7047^{*}$ & 7415 & 51.9 & & \\
\hline & $20^{-}$ & 7564 & & 48.5 & & \\
\hline & $21^{-}$ & $8013^{*}$ & & 42.9 & & \\
\hline \multirow[t]{14}{*}{${ }_{61}^{136} \mathrm{Pm}_{75}$} & $8^{+}$ & $0^{*}$ & & & & {$[86,102,104,129,141,142]$} \\
\hline & $9^{+}$ & $99^{*}$ & & & & \\
\hline & $10^{+}$ & $267^{*}$ & & & & \\
\hline & $11^{+}$ & $552^{*}$ & 858 & $9.8\left(\begin{array}{l}+4.3 \\
-4.3\end{array}\right)$ & & \\
\hline & $12^{+}$ & $844^{*}$ & 1146 & $5.5\left(\begin{array}{l}+0.7 \\
-0.7\end{array}\right)$ & & \\
\hline & $13^{+}$ & $1252^{*}$ & 1510 & $5.1\left({ }_{-0.7}^{+0.7}\right)$ & $6.1\left({ }_{-1.7}^{+1.7}\right)$ & \\
\hline & $14^{+}$ & $1608^{*}$ & 1936 & $2.6\left(\begin{array}{l}+0.3 \\
-0.3\end{array}\right)$ & $5.7\left(\begin{array}{c}+1.5 \\
-1.5\end{array}\right)$ & \\
\hline & $15^{+}$ & $2086^{*}$ & 2360 & $3.8\left(\begin{array}{l}+0.9 \\
-0.9\end{array}\right)$ & & \\
\hline & $16^{+}$ & $2504^{*}$ & 2819 & $1.0\left(\begin{array}{l}+0.4 \\
-0.4\end{array}\right)$ & & \\
\hline & $17^{+}$ & $3010^{*}$ & 3254 & $1.8\left({ }_{-0.6}^{+0.6}\right)$ & & \\
\hline & $18^{+}$ & $3479^{*}$ & 3654 & $2.1\left(\begin{array}{l}+3.6 \\
-3.6\end{array}\right)$ & & \\
\hline & $19^{+}$ & $3935^{*}$ & 4088 & & & \\
\hline & $20^{+}$ & $4377^{*}$ & 4469 & & & \\
\hline & $21^{+}$ & $4814^{*}$ & 4872 & & & \\
\hline \multirow[t]{10}{*}{${ }_{61}^{138} \mathrm{Pm}_{77}$} & $9^{+}$ & $585^{*}$ & & & & {$[103]$} \\
\hline & $10^{+}$ & $706^{*}$ & & & & \\
\hline & $11^{+}$ & $1063^{*}$ & 1319 & & & \\
\hline & $12^{+}$ & $1413^{*}$ & 1619 & $3.46\left(\begin{array}{l}+0.38 \\
-0.38\end{array}\right)$ & & \\
\hline & $13^{+}$ & $1890^{*}$ & 2097 & $3.81\left({ }_{-0.34}^{+0.34}\right)$ & $3.2\left({ }_{-0.5}^{+0.5}\right)$ & \\
\hline & $14^{+}$ & $2283^{*}$ & 2506 & $2.90\left(\begin{array}{l}+0.38 \\
-0.38\end{array}\right)$ & $2.4\left(\begin{array}{l}+0.5 \\
-0.5\end{array}\right)$ & \\
\hline & $15^{+}$ & $2829^{*}$ & 3077 & $2.35\left({ }_{-0.33}^{+0.33}\right)$ & $2.5\left(\begin{array}{c}+0.9 \\
-0.9\end{array}\right)$ & \\
\hline & $16^{+}$ & 3279 & & $1.74\left(\begin{array}{l}+0.40 \\
-0.40\end{array}\right)$ & & \\
\hline & $17^{+}$ & 3855 & & $1.83\left({ }_{-0.55}^{+0.55}\right)$ & & \\
\hline & $18^{+}$ & 4340 & & & & \\
\hline \multirow[t]{3}{*}{${ }_{63}^{138} \mathrm{Eu}_{75}$} & $8^{+}$ & 0 & & & & {$[104,141,142]$} \\
\hline & $9^{+}$ & $104^{*}$ & & & & \\
\hline & $10^{+}$ & $271^{*}$ & 628 & & & \\
\hline
\end{tabular}


Table 1 (continued)

\begin{tabular}{|c|c|c|c|c|c|c|}
\hline \multirow{2}{*}{ Nuclei } & \multirow{2}{*}{$I^{\pi}(\hbar)$} & \multicolumn{2}{|c|}{$E(\mathrm{keV})$} & \multicolumn{2}{|c|}{$B(M 1) / B(E 2)\left(\mu_{N}^{2} / \mathrm{e}^{2} \mathrm{~b}^{2}\right)$} & \multirow{2}{*}{ References } \\
\hline & & yrast & side & yrast & side & \\
\hline & $11^{+}$ & $544^{*}$ & 792 & $3.1\left(\begin{array}{l}+0.4 \\
-0.4\end{array}\right)$ & & \\
\hline & $12^{+}$ & $806^{*}$ & 1091 & $2.3\left({ }_{-0.1}^{+0.1}\right)$ & $3.1\left(\left(_{-0.2}^{+0.2}\right)\right.$ & \\
\hline & $13^{+}$ & $1168^{*}$ & 1442 & $2.5\left(\begin{array}{l}+0.3 \\
-0.3\end{array}\right)$ & $4.0\left(\begin{array}{l}+1.0 \\
-1.0\end{array}\right)$ & \\
\hline & $14^{+}$ & $1488^{*}$ & 1847 & $1.6\left(\begin{array}{l}+0.2 \\
-0.2\end{array}\right)$ & & \\
\hline & $15^{+}$ & $1916^{*}$ & 2230 & $2.1\left(\begin{array}{l}+0.6 \\
-0.6\end{array}\right)$ & & \\
\hline & $16^{+}$ & $2298^{*}$ & 2708 & $1.3\left({ }_{-0.2}^{+0.2}\right)$ & & \\
\hline & $17^{+}$ & $2761^{*}$ & 3128 & $2.2\left(\begin{array}{l}+0.5 \\
-0.5\end{array}\right)$ & & \\
\hline & $18^{+}$ & $3177^{*}$ & & $1.4\left(\begin{array}{c}+0.4 \\
-0.4\end{array}\right)$ & & \\
\hline & $19^{+}$ & $3589^{*}$ & & $3.4\left(\begin{array}{l}+0.9 \\
-0.9\end{array}\right)$ & & \\
\hline & $20^{+}$ & $4015^{*}$ & & $3.4\left(\begin{array}{l}+0.7 \\
-0.7\end{array}\right)$ & & \\
\hline & $21^{+}$ & $4456^{*}$ & & $3.8\left(\begin{array}{l}+0.7 \\
-0.7\end{array}\right)$ & & \\
\hline & $22^{+}$ & $4982^{*}$ & & $1.7\left(\begin{array}{l}+0.8 \\
-0.8\end{array}\right)$ & & \\
\hline & $23^{+}$ & 5486 & & & & \\
\hline & $24^{+}$ & $6025^{*}$ & & & & \\
\hline & $25^{+}$ & $6632^{*}$ & & & & \\
\hline \multirow[t]{19}{*}{${ }_{63}^{140} \mathrm{Eu}_{77}$} & $5^{-}$ & $0.0^{*}$ & & & & {$[105,142]$} \\
\hline & $6^{-}$ & $170.6^{*}$ & 285.4 & & & \\
\hline & $7^{-}$ & $361.7^{*}$ & & $0.94\left(\begin{array}{l}+0.17 \\
-0.17\end{array}\right)$ & & \\
\hline & $8^{-}$ & 654.6 & 763.1 & $0.00\left(\begin{array}{l}+0.08 \\
-0.08\end{array}\right)$ & & \\
\hline & $9^{-}$ & 898.6 & & $0.41\left({ }_{-0.31}^{+0.31}\right)$ & & \\
\hline & $10^{-}$ & 1376.6 & 1364.8 & & & \\
\hline & $11^{-}$ & 1614.2 & & & & \\
\hline & $12^{-}$ & 1959.6 & 2197.1 & & & \\
\hline & $13^{-}$ & 2444.2 & 2427.5 & & & \\
\hline & $14^{-}$ & 2884.6 & 2597.5 & & & \\
\hline & $15^{-}$ & & 2970.4 & & & \\
\hline & $16^{-}$ & & 3424.4 & & & \\
\hline & $17^{-}$ & & 3884.4 & & & \\
\hline & $18^{-}$ & & 4264.0 & & & \\
\hline & $8^{+}$ & & 53.0 & & & {$[105,142]$} \\
\hline & $9^{+}$ & 0.0 & 147.9 & & & \\
\hline & $10^{+}$ & 71.0 & & & & \\
\hline & $11^{+}$ & 436.8 & 554.5 & & & \\
\hline & $12^{+}$ & 711.5 & & $0.40\left(\begin{array}{l}+0.11 \\
-0.11\end{array}\right)$ & & \\
\hline
\end{tabular}


Table 1 (continued)

\begin{tabular}{|c|c|c|c|c|c|c|}
\hline \multirow{2}{*}{ Nuclei } & \multirow{2}{*}{$I^{\pi}(\hbar)$} & \multicolumn{2}{|c|}{$E(\mathrm{keV})$} & \multicolumn{2}{|c|}{$B(M 1) / B(E 2)\left(\mu_{N}^{2} / \mathrm{e}^{2} \mathrm{~b}^{2}\right)$} & \multirow{2}{*}{ References } \\
\hline & & yrast & side & yrast & side & \\
\hline & $13^{+}$ & 1157.5 & 1201.9 & $3.42\left(\begin{array}{l}+0.90 \\
-0.90\end{array}\right)$ & & \\
\hline & $14^{+}$ & 1518.8 & & $1.53\left({ }_{-0.89}^{+0.89}\right)$ & & \\
\hline & $15^{+}$ & 1989.3 & 2020.9 & $1.97\left(\begin{array}{l}+0.39 \\
-0.39\end{array}\right)$ & & \\
\hline & $16^{+}$ & 2438.8 & & & & \\
\hline & $17^{+}$ & 2500.2 & 2636.9 & & & \\
\hline & $19^{+}$ & 3147.9 & & & & \\
\hline & $21^{+}$ & 3902.1 & & & & \\
\hline & $23^{+}$ & 4809.5 & & & & \\
\hline & $25^{+}$ & 5801.2 & & & & \\
\hline \multirow[t]{9}{*}{${ }_{77}^{188} \operatorname{Ir}_{111}$} & $9^{-}$ & $923.7^{*}$ & & & & {$[106]$} \\
\hline & $10^{-}$ & $1222.3^{*}$ & & & & \\
\hline & $11^{-}$ & $1398.9^{*}$ & & $0.90\left({ }_{-0.04}^{+0.04}\right)$ & & \\
\hline & $12^{-}$ & $1711.2^{*}$ & & $1.80\left(\begin{array}{l}+0.05 \\
-0.05\end{array}\right)$ & & \\
\hline & $13^{-}$ & $1922.8^{*}$ & 2168.2 & $0.64\left(\begin{array}{l}+0.03 \\
-0.03\end{array}\right)$ & & \\
\hline & $14^{-}$ & $2290.1^{*}$ & 2443.6 & $1.13\left(\begin{array}{l}+0.05 \\
-0.05\end{array}\right)$ & & \\
\hline & $15^{-}$ & $2556.7^{*}$ & 2679.7 & $0.58\left(\begin{array}{l}+0.03 \\
-0.03\end{array}\right)$ & $1.25\left(\begin{array}{c}+0.12 \\
-0.12\end{array}\right)$ & \\
\hline & $16^{-}$ & 2990.5 & 2945.3 & $0.77\left(\begin{array}{l}+0.06 \\
-0.06\end{array}\right)$ & $1.28\left(\begin{array}{c}+0.18 \\
-0.18\end{array}\right)$ & \\
\hline & $17^{-}$ & & 3201.7 & & & \\
\hline \multirow[t]{8}{*}{${ }_{81}^{193} \mathrm{Tl}_{112}$} & $13.5^{-}$ & 3092 & & & & {$[107]$} \\
\hline & $14.5^{-}$ & 3251 & 3402 & & & \\
\hline & $15.5^{-}$ & 3402 & 3684 & & & \\
\hline & $16.5^{-}$ & 3624 & 3883 & & & \\
\hline & $17.5^{-}$ & 3862 & 4253 & & & \\
\hline & $18.5^{-}$ & 4188 & 4505 & & & \\
\hline & $19.5^{-}$ & 4525 & 4874 & & & \\
\hline & $20.5^{-}$ & 4868 & 5230 & & & \\
\hline \multirow[t]{8}{*}{${ }_{81}^{194} \mathrm{Tl}_{113}$} & $8^{-}$ & 293 & & & & {$[108,143,144]$} \\
\hline & $9^{-}$ & 338 & & & & \\
\hline & $10^{-}$ & 434 & & & & \\
\hline & $11^{-}$ & 712 & 1175 & & & \\
\hline & $12^{-}$ & 957 & 1481 & & & \\
\hline & $13^{-}$ & 1361 & 1738 & & & \\
\hline & $14^{-}$ & 1644 & 2001 & & & \\
\hline & $15^{-}$ & 2112 & 2344 & & & \\
\hline
\end{tabular}


Table 1 (continued)

\begin{tabular}{|c|c|c|c|c|c|c|}
\hline \multirow{2}{*}{ Nuclei } & \multirow{2}{*}{$I^{\pi}(\hbar)$} & \multicolumn{2}{|c|}{$E(\mathrm{keV})$} & \multicolumn{2}{|c|}{$B(M 1) / B(E 2)\left(\mu_{N}^{2} / \mathrm{e}^{2} \mathrm{~b}^{2}\right)$} & \multirow{2}{*}{ References } \\
\hline & & yrast & side & yrast & side & \\
\hline & $16^{-}$ & 2404 & 2682 & & & \\
\hline & $17^{-}$ & 2882 & 3003 & & & \\
\hline & $18^{-}$ & 3130 & 3257 & & & \\
\hline & $19^{-}$ & 3381 & 3427 & & & \\
\hline & $20^{-}$ & 3518 & 3628 & & & \\
\hline & $21^{-}$ & 3840 & 3877 & $4.45\left(\begin{array}{l}+2.92 \\
-1.83\end{array}\right)$ & & \\
\hline & $22^{-}$ & 4081 & 4181 & $2.46\left({ }_{-0.77}^{+1.39}\right)$ & $6.17\left(\begin{array}{l}+5.01 \\
-2.34\end{array}\right)$ & \\
\hline & $23^{-}$ & 4462 & 4560 & $7.18\left(\begin{array}{l}+5.89 \\
-2.61\end{array}\right)$ & 4.03 & \\
\hline & $24^{-}$ & 4824 & & $3.66\left(\begin{array}{l}+3.61 \\
-2.06\end{array}\right)$ & & \\
\hline \multirow[t]{11}{*}{${ }_{81}^{198} \mathrm{Tl}_{117}$} & $8^{-}$ & 391 & & & & {$[109,145]$} \\
\hline & $9^{-}$ & 463 & & & & \\
\hline & $10^{-}$ & 585 & 1111 & & & \\
\hline & $11^{-}$ & $844^{*}$ & 1293 & $3.8\left({ }_{-0.4}^{+0.4}\right)$ & & \\
\hline & $12^{-}$ & $1091^{*}$ & 1541 & $4.1\left(\begin{array}{c}+0.5 \\
-0.5\end{array}\right)$ & & \\
\hline & $13^{-}$ & $1493^{*}$ & 1857 & $3.9\left(\begin{array}{l}+0.5 \\
-0.5\end{array}\right)$ & $4.4\left(\begin{array}{l}+1.4 \\
-1.4\end{array}\right)$ & \\
\hline & $14^{-}$ & $1790^{*}$ & 2294 & $3.1\left({ }_{-0.6}^{+0.6}\right)$ & $2.5\left(\begin{array}{l}+1.2 \\
-1.2\end{array}\right)$ & \\
\hline & $15^{-}$ & 2278 & & $4.3\left(\begin{array}{l}+0.9 \\
-0.9\end{array}\right)$ & & \\
\hline & $16^{-}$ & 2552 & & $6.4\left(\begin{array}{l}+2.1 \\
-2.1\end{array}\right)$ & & \\
\hline & $17^{-}$ & 2947 & & $1.7\left(\begin{array}{l}+0.8 \\
-0.8\end{array}\right)$ & & \\
\hline & $18^{-}$ & 3219 & & & & \\
\hline
\end{tabular}




\section{Table 2}

Chiral doublet bands with $B(M 1)$ and $B(E 2)$ values. See page 44 for Explanation of Tables for details.

\begin{tabular}{|c|c|c|c|c|c|c|c|c|}
\hline \multirow{2}{*}{ Nuclei } & \multirow{2}{*}{$I^{\pi}(\hbar)$} & \multicolumn{2}{|c|}{$E(\mathrm{keV})$} & \multicolumn{2}{|c|}{$B(M 1)\left(\mu_{N}^{2}\right)$} & \multicolumn{2}{|c|}{$B(E 2)\left(\mathrm{e}^{2} \mathrm{~b}^{2}\right)$} & \multirow{2}{*}{ References } \\
\hline & & yrast & side & yrast & side & yrast & side & \\
\hline \multirow[t]{32}{*}{${ }_{45}^{103} \mathrm{Rh}_{58}$} & $10.5^{+}$ & 3238 & & & & & & {$[54,117,118]$} \\
\hline & $11.5^{+}$ & 3357 & & & & & & \\
\hline & $12.5^{+}$ & 3591 & & & & & & \\
\hline & $13.5^{+}$ & 3899 & 4445 & & & & & \\
\hline & $14.5^{+}$ & 4281 & 4789 & & & & & \\
\hline & $15.5^{+}$ & 4665 & 5166 & & & & & \\
\hline & $16.5^{+}$ & 5156 & 5616 & & & & & \\
\hline & $17.5^{+}$ & 5622 & 6062 & & & & & \\
\hline & $18.5^{+}$ & 6163 & 6528 & & & & & \\
\hline & $19.5^{+}$ & 6706 & 7074 & & & & & \\
\hline & $20.5^{+}$ & 7317 & & & & & & \\
\hline & $21.5^{+}$ & 7952 & & & & & & \\
\hline & $6.5^{-}$ & 2033 & & & & & & {$[54,117,118]$} \\
\hline & $7.5^{-}$ & 2219 & & & & & & \\
\hline & $8.5^{-}$ & 2343 & & & & & & \\
\hline & $9.5^{-}$ & 2538 & 2744 & & & & & \\
\hline & $10.5^{-}$ & 2751 & 2934 & & & & & \\
\hline & $11.5^{-}$ & $3011^{*}$ & 3273 & & & & & \\
\hline & $12.5^{-}$ & $3327^{*}$ & 3615 & $2.3\left(\begin{array}{c}+0.4 \\
-0.4\end{array}\right)$ & & $0.077\left({ }_{-0.014}^{+0.014}\right)$ & & \\
\hline & $13.5^{-}$ & $3769^{*}$ & 4080 & $1.8\left({ }_{-0.2}^{+0.2}\right)$ & & $0.14\left(\begin{array}{l}+0.03 \\
-0.03\end{array}\right)$ & & \\
\hline & $14.5^{-}$ & 4195 & 4559 & $1.2\left(\begin{array}{c}+0.4 \\
-0.4\end{array}\right)$ & & $0.11\left(\begin{array}{l}+0.04 \\
-0.04\end{array}\right)$ & & \\
\hline & $15.5^{-}$ & 4763 & 5091 & & & & & \\
\hline & $16.5^{-}$ & 5297 & 5685 & & & & & \\
\hline & $17.5^{-}$ & 5923 & & & & & & \\
\hline & $18.5^{-}$ & 6586 & & & & & & \\
\hline & $19.5^{-}$ & 7186 & & & & & & \\
\hline & $6.5^{-}$ & 2228 & & & & & & {$[54,117,118]$} \\
\hline & $7.5^{-}$ & 2366 & 2443 & & & & & \\
\hline & $8.5^{-}$ & 2520 & 2643 & & & & & \\
\hline & $9.5^{-}$ & 2699 & 2871 & & & & & \\
\hline & $10.5^{-}$ & 2915 & 3090 & & & & & \\
\hline & $11.5^{-}$ & 3227 & 3416 & & & & & \\
\hline
\end{tabular}


Table 2 (continued)

\begin{tabular}{|c|c|c|c|c|c|c|c|c|}
\hline \multirow{2}{*}{ Nuclei } & \multirow{2}{*}{$I^{\pi}(\hbar)$} & \multicolumn{2}{|c|}{$E(\mathrm{keV})$} & \multicolumn{2}{|c|}{$B(M 1)\left(\mu_{N}^{2}\right)$} & \multicolumn{2}{|c|}{$B(E 2)\left(\mathrm{e}^{2} \mathrm{~b}^{2}\right)$} & \multirow{2}{*}{ References } \\
\hline & & yrast & side & yrast & side & yrast & side & \\
\hline & $12.5^{-}$ & 3668 & 3778 & & & & & \\
\hline & $13.5^{-}$ & 4106 & 4210 & & & & & \\
\hline & $14.5^{-}$ & 4606 & 4659 & & & & & \\
\hline & $15.5^{-}$ & 5061 & 5059 & & & & & \\
\hline & $16.5^{-}$ & 5577 & & & & & & \\
\hline & $17.5^{-}$ & 6143 & & & & & & \\
\hline \multirow[t]{9}{*}{${ }_{45}^{104} \mathrm{Rh}_{59}$} & $9^{-}$ & $483^{*}$ & & $2.3\left(\begin{array}{l}+0.2 \\
-0.2\end{array}\right)$ & & & & {$[80,118]$} \\
\hline & $10^{-}$ & $840^{*}$ & 1228 & $0.95\left(\begin{array}{l}+0.08 \\
-0.08\end{array}\right)$ & & $0.063\left({ }_{-0.017}^{+0.017}\right)$ & & \\
\hline & $11^{-}$ & $1168^{*}$ & 1582 & $0.91\left(\begin{array}{l}+0.12 \\
-0.12\end{array}\right)$ & & $0.093\left({ }_{-0.020}^{+0.020}\right)$ & & \\
\hline & $12^{-}$ & $1636^{*}$ & 1971 & $0.44\left(\begin{array}{l}+0.06 \\
-0.06\end{array}\right)$ & & $0.039\left({ }_{-0.010}^{+0.010}\right)$ & & \\
\hline & $13^{-}$ & $2111^{*}$ & 2370 & $0.42\left(\begin{array}{l}+0.15 \\
-0.15\end{array}\right)$ & & $0.067\left({ }_{-0.025}^{+0.025}\right)$ & & \\
\hline & $14^{-}$ & $2639^{*}$ & 2834 & & & & & \\
\hline & $15^{-}$ & $3229^{*}$ & 3320 & & & & & \\
\hline & $16^{-}$ & $3800^{*}$ & 3876 & & & & & \\
\hline & $17^{-}$ & 4406 & 4406 & & & & & \\
\hline \multirow[t]{13}{*}{${ }_{47}^{106} \mathrm{Ag}_{59}$} & $10^{-}$ & 2271.7 & 3203.8 & & & & & {$[85,120-124]$} \\
\hline & $11^{-}$ & 2441.4 & 3423.5 & & & & & \\
\hline & $12^{-}$ & 2660.4 & 3676.0 & & & & & \\
\hline & $13^{-}$ & 2930.2 & 3941.6 & & & & & \\
\hline & $14^{-}$ & 3256.7 & 4263.7 & $1.335\left({ }_{-0.397}^{+0.397}\right)$ & & $0.100\left({ }_{-0.043}^{+0.043}\right)$ & & \\
\hline & $15^{-}$ & 3686.1 & 4636.6 & $0.968\left({ }_{-0.211}^{+0.211}\right)$ & $3.164\left({ }_{-0.873}^{+0.873}\right)$ & $0.106\left({ }_{-0.020}^{+0.020}\right)$ & $0.271\left({ }_{-0.084}^{+0.084}\right)$ & \\
\hline & $16^{-}$ & $4223.3^{*}$ & 5051.7 & $0.539\left({ }_{-0.096}^{+0.096}\right)$ & $2.041\left({ }_{-0.317}^{+0.317}\right)$ & $0.099\left({ }_{-0.015}^{+0.015}\right)$ & $0.232\left({ }_{-0.050}^{+0.050}\right)$ & \\
\hline & $17^{-}$ & $4742.6^{*}$ & 5561.2 & $0.463 \quad\left(\begin{array}{l}+0.010 \\
-0.010\end{array}\right)$ & $1.050\left({ }_{-0.221}^{+0.221}\right)$ & $0.101\left({ }_{-0.019}^{+0.019}\right)$ & $0.153\left({ }_{-0.039}^{+0.039}\right)$ & \\
\hline & $18^{-}$ & $5414.9^{*}$ & 6065.8 & $0.635\left({ }_{-0.251}^{+0.251}\right)$ & $1.291\left({ }_{-0.327}^{+0.327}\right)$ & $0.085\left({ }_{-0.032}^{+0.032}\right)$ & $0.202\left({ }_{-0.043}^{+0.043}\right)$ & \\
\hline & $19^{-}$ & $6026.8^{*}$ & 6691.0 & $0.290\left({ }_{-0.054}^{+0.054}\right)$ & $0.739\left({ }_{-0.202}^{+0.202}\right)$ & $0.051\left({ }_{-0.009}^{+0.009}\right)$ & $0.136\left({ }_{-0.034}^{+0.034}\right)$ & \\
\hline & $20^{-}$ & $6760.7^{*}$ & 7276.7 & $0.289\left({ }_{-0.072}^{+0.072}\right)$ & $0.517\left(\left(_{-0.167}^{+0.167}\right)\right.$ & $0.043\left({ }_{-0.011}^{+0.011}\right)$ & $0.079\left({ }_{-0.026}^{+0.026}\right)$ & \\
\hline & $21^{-}$ & 7531.7 & 7945.6 & $0.065\left(\begin{array}{l}+0.028 \\
-0.028\end{array}\right)$ & & $0.035\left({ }_{-0.014}^{+0.014}\right)$ & & \\
\hline & $22^{-}$ & 8192.5 & & & & $0.047\left({ }_{-0.010}^{+0.010}\right)$ & & \\
\hline \multirow[t]{6}{*}{${ }_{47}^{107} \mathrm{Ag}_{60}$} & $10.5^{-}$ & $2620.8^{*}$ & 2901.6 & & & & & {$[60,125,126]$} \\
\hline & $11.5^{-}$ & $2928.7^{*}$ & 3091.8 & $1.25\left(\begin{array}{l}+0.36 \\
-0.36\end{array}\right)$ & & $0.18\left({ }_{-0.06}^{+0.06}\right)$ & & \\
\hline & $12.5^{-}$ & $3338.7^{*}$ & 3392.0 & $0.82\left(\begin{array}{c}+0.18 \\
-0.18\end{array}\right)$ & & $0.09\left({ }_{-0.02}^{+0.02}\right)$ & & \\
\hline & $13.5^{-}$ & 3800.0 & 3897.5 & $0.85\left(\begin{array}{l}+0.17 \\
-0.17\end{array}\right)$ & & $0.08\left(\begin{array}{l}+0.02 \\
-0.02\end{array}\right)$ & & \\
\hline & $14.5^{-}$ & 4270.0 & 4349.6 & $>0.39$ & & $>0.06$ & & \\
\hline & $15.5^{-}$ & 4878.8 & 4932.0 & & & & & \\
\hline
\end{tabular}


Table 2 (continued)

\begin{tabular}{|c|c|c|c|c|c|c|c|c|}
\hline \multirow{2}{*}{ Nuclei } & \multirow{2}{*}{$I^{\pi}(\hbar)$} & \multicolumn{2}{|c|}{$E(\mathrm{keV})$} & \multicolumn{2}{|c|}{$B(M 1)\left(\mu_{N}^{2}\right)$} & \multicolumn{2}{|c|}{$B(E 2)\left(\mathrm{e}^{2} \mathrm{~b}^{2}\right)$} & \multirow{2}{*}{ References } \\
\hline & & yrast & side & yrast & side & yrast & side & \\
\hline & $16.5^{-}$ & 5437.3 & & & & & & \\
\hline & $11.5^{+}$ & 3334.8 & & & & & & {$[60,125,126]$} \\
\hline & $12.5^{+}$ & 3556.7 & & $5.2\left(\begin{array}{l}+2.3 \\
-2.3\end{array}\right)$ & & $0.15\left(\begin{array}{l}+0.07 \\
-0.07\end{array}\right)$ & & \\
\hline & $13.5^{+}$ & 3851.3 & & $2.7\left(\begin{array}{l}+0.6 \\
-0.6\end{array}\right)$ & & $0.23\left(\begin{array}{l}+0.05 \\
-0.05\end{array}\right)$ & & \\
\hline & $14.5^{+}$ & $4230.0^{*}$ & 4841.7 & $1.8\left(\begin{array}{l}+0.3 \\
-0.3\end{array}\right)$ & & $0.15\left(\begin{array}{l}+0.03 \\
-0.03\end{array}\right)$ & & \\
\hline & $15.5^{+}$ & $4626.4^{*}$ & 5131.1 & $1.9\left(\begin{array}{l}+0.3 \\
-0.3\end{array}\right)$ & & $0.14\left(\begin{array}{l}+0.03 \\
-0.03\end{array}\right)$ & & \\
\hline & $16.5^{+}$ & $5120.5^{*}$ & 5448.9 & $1.9\left(\begin{array}{l}+0.4 \\
-0.4\end{array}\right)$ & & $0.23\left(\begin{array}{l}+0.06 \\
-0.06\end{array}\right)$ & & \\
\hline & $17.5^{+}$ & $5621.5^{*}$ & 5818.4 & $>1.25$ & & $>0.16$ & & \\
\hline & $18.5^{+}$ & $6192.8^{*}$ & 6250.2 & & & & & \\
\hline & $19.5^{+}$ & 6785.9 & $6761.3^{*}$ & & & & & \\
\hline & $20.5^{+}$ & 7441.1 & $7315.5^{*}$ & & & & & \\
\hline & $21.5^{+}$ & & $7919.9^{*}$ & & & & & \\
\hline & $22.5^{+}$ & & $8591.2^{*}$ & & & & & \\
\hline \multirow[t]{18}{*}{${ }_{55}^{124} \mathrm{Cs}_{69}$} & $8^{+}$ & $561.9^{*}$ & & & & & & {$[90]$} \\
\hline & $9^{+}$ & $619.9^{*}$ & & & & & & \\
\hline & $10^{+}$ & $743.9^{*}$ & & & & & & \\
\hline & $11^{+}$ & $1055.9^{*}$ & 1260.2 & & & & & \\
\hline & $12^{+}$ & $1275.9^{*}$ & 1631.2 & & & & & \\
\hline & $13^{+}$ & $1673.9^{*}$ & 1893.2 & & & & & \\
\hline & $14^{+}$ & $1989.9^{*}$ & 2265.2 & $0.27\left(\begin{array}{l}+0.05 \\
-0.07\end{array}\right)$ & $0.16\left(\begin{array}{l}+0.05 \\
-0.05\end{array}\right)$ & $0.31\left(\begin{array}{l}+0.06 \\
-0.08\end{array}\right)$ & $0.12\left(\begin{array}{l}+0.03 \\
-0.04\end{array}\right)$ & \\
\hline & $15^{+}$ & $2446.9^{*}$ & 2666.2 & $0.41\left(\begin{array}{l}+0.09 \\
-0.13\end{array}\right)$ & $0.66\left(\begin{array}{c}+0.16 \\
-0.14\end{array}\right)$ & $0.12\left(\begin{array}{c}+0.03 \\
-0.04\end{array}\right)$ & $0.25\left(\begin{array}{l}+0.07 \\
-0.06\end{array}\right)$ & \\
\hline & $16^{+}$ & $2858.9^{*}$ & 3090.2 & $\leq 0.05$ & $\leq 0.05$ & $0.24\left(\begin{array}{l}+0.06 \\
-0.06\end{array}\right)$ & $0.13\left(\begin{array}{l}+0.05 \\
-0.05\end{array}\right)$ & \\
\hline & $17^{+}$ & $3344.9^{*}$ & 3574.2 & $0.41\left(\begin{array}{l}+0.11 \\
-0.07\end{array}\right)$ & $0.34\left(\begin{array}{l}+0.11 \\
-0.09\end{array}\right)$ & $0.14\left({ }_{-0.03}^{+0.04}\right)$ & $0.21\left(\begin{array}{l}+0.07 \\
-0.06\end{array}\right)$ & \\
\hline & $18^{+}$ & $3832.9^{*}$ & & $\leq 0.05$ & & $0.19\left(\begin{array}{l}+0.04 \\
-0.04\end{array}\right)$ & & \\
\hline & $19^{+}$ & $4342.9^{*}$ & & $0.47\left(\begin{array}{l}+0.07 \\
-0.09\end{array}\right)$ & & $0.15\left(\begin{array}{l}+0.03 \\
-0.03\end{array}\right)$ & & \\
\hline & $20^{+}$ & $4906.9^{*}$ & & $\leq 0.05$ & & $0.19\left(\begin{array}{l}+0.05 \\
-0.05\end{array}\right)$ & & \\
\hline & $21^{+}$ & $5424.9^{*}$ & & $0.38\left(\begin{array}{l}+0.16 \\
-0.09\end{array}\right)$ & & $0.15\left(\begin{array}{l}+0.04 \\
-0.06\end{array}\right)$ & & \\
\hline & $22^{+}$ & $6086.9^{*}$ & & $\leq 0.02$ & & $0.06\left({ }_{-0.01}^{+0.01}\right)$ & & \\
\hline & $23^{+}$ & 6550.9 & & $0.16\left(\begin{array}{l}+0.04 \\
-0.04\end{array}\right)$ & & $0.07\left(\begin{array}{l}+0.01 \\
-0.01\end{array}\right)$ & & \\
\hline & $24^{+}$ & 7357.9 & & & & & & \\
\hline & $25^{+}$ & 7649.9 & & & & & & \\
\hline \multirow[t]{3}{*}{${ }_{55}^{126} \mathrm{Css}_{71}$} & $9^{+}$ & 0 & & & & & & {$[91,127,128]$} \\
\hline & $10^{+}$ & 140 & & & & & & \\
\hline & $11^{+}$ & 477 & 637 & & & & & \\
\hline
\end{tabular}


Table 2 (continued)

\begin{tabular}{|c|c|c|c|c|c|c|c|c|}
\hline \multirow{2}{*}{ Nuclei } & \multirow{2}{*}{$I^{\pi}(\hbar)$} & \multicolumn{2}{|c|}{$E(\mathrm{keV})$} & \multicolumn{2}{|c|}{$B(M 1)\left(\mu_{N}^{2}\right)$} & \multicolumn{2}{|c|}{$B(E 2)\left(\mathrm{e}^{2} \mathrm{~b}^{2}\right)$} & \multirow{2}{*}{ References } \\
\hline & & yrast & side & yrast & side & yrast & side & \\
\hline & $12^{+}$ & 732 & 999 & & & & & \\
\hline & $13^{+}$ & 1128 & 1326 & & & & & \\
\hline & $14^{+}$ & 1471 & 1671 & $0.14\left(\begin{array}{l}+0.07 \\
-0.02\end{array}\right)$ & $0.16\left(\begin{array}{l}+0.05 \\
-0.02\end{array}\right)$ & $0.23\left({ }_{-0.03}^{+0.11}\right)$ & $0.13\left(\begin{array}{l}+0.05 \\
-0.02\end{array}\right)$ & \\
\hline & $15^{+}$ & 1935 & 2097 & $0.47\left(\begin{array}{l}+0.27 \\
-0.07\end{array}\right)$ & $0.25\left(\begin{array}{l}+0.16 \\
-0.05\end{array}\right)$ & $0.11\left({ }_{-0.02}^{+0.06}\right)$ & $0.08\left(\begin{array}{l}+0.05 \\
-0.02\end{array}\right)$ & \\
\hline & $16^{+}$ & 2350 & 2572 & $\leq 0.04$ & $\leq 0.07$ & $0.14\left(\begin{array}{l}+0.05 \\
-0.02\end{array}\right)$ & $0.05\left(\begin{array}{l}+0.03 \\
-0.01\end{array}\right)$ & \\
\hline & $17^{+}$ & 2846 & 3033 & $0.23\left(\begin{array}{l}+0.11 \\
-0.04\end{array}\right)$ & $0.11\left(\begin{array}{l}+0.05 \\
-0.02\end{array}\right)$ & $0.08\left({ }_{-0.01}^{+0.04}\right)$ & $0.12\left(\begin{array}{l}+0.05 \\
-0.02\end{array}\right)$ & \\
\hline & $18^{+}$ & 3311 & & $\leq 0.07$ & & $0.15\left(\begin{array}{l}+0.05 \\
-0.02\end{array}\right)$ & & \\
\hline & $19^{+}$ & 3839 & 4040 & $0.34\left(\begin{array}{l}+0.23 \\
-0.07\end{array}\right)$ & & $0.09\left({ }_{-0.02}^{+0.06}\right)$ & $0.07\left({ }_{-0.01}^{+0.03}\right)$ & \\
\hline & $20^{+}$ & 4357 & & $\leq 0.07$ & & $0.09\left(\begin{array}{l}+0.04 \\
-0.01\end{array}\right)$ & & \\
\hline & $21^{+}$ & 4911 & & $0.18\left(\begin{array}{l}+0.18 \\
-0.07\end{array}\right)$ & & $0.07\left(\begin{array}{l}+0.07 \\
-0.02\end{array}\right)$ & & \\
\hline \multirow[t]{16}{*}{${ }_{55}^{130} \mathrm{Cs}_{75}$} & $10^{+}$ & $959.7^{*}$ & & & & & & {$[24,86,93,129-131]$} \\
\hline & $11^{+}$ & $1312.7^{*}$ & 1506.4 & & & & & \\
\hline & $12^{+}$ & $1602.4^{*}$ & 1907.7 & $0.70\left(\begin{array}{l}+0.22 \\
-0.17\end{array}\right)$ & & $0.17\left(\left(_{-0.04}^{+0.05}\right)\right.$ & & \\
\hline & $13^{+}$ & $2019.0^{*}$ & 2280.7 & $0.54\left(\begin{array}{l}+0.11 \\
-0.12\end{array}\right)$ & $0.32\left(\begin{array}{l}+0.15 \\
-0.11\end{array}\right)$ & $0.08\left({ }_{-0.02}^{+0.02}\right)$ & $0.15\left(\begin{array}{l}+0.07 \\
-0.05\end{array}\right)$ & \\
\hline & $14^{+}$ & $2445.1^{*}$ & 2632.0 & $0.24\left(\begin{array}{l}+0.10 \\
-0.07\end{array}\right)$ & $0.20\left(\begin{array}{c}+0.04 \\
-0.04\end{array}\right)$ & $0.14\left(\begin{array}{l}+0.05 \\
-0.04\end{array}\right)$ & $0.08\left(\begin{array}{l}+0.02 \\
-0.02\end{array}\right)$ & \\
\hline & $15^{+}$ & $2913.5^{*}$ & 3085.0 & $0.92\left(\begin{array}{c}+0.54 \\
-0.40\end{array}\right)$ & $1.49\left(\begin{array}{l}+1.05 \\
-0.69\end{array}\right)$ & $0.19\left(\begin{array}{l}+0.11 \\
-0.08\end{array}\right)$ & $0.19\left(\begin{array}{c}+0.13 \\
-0.08\end{array}\right)$ & \\
\hline & $16^{+}$ & $3379.1^{*}$ & 3563.4 & $0.12\left(\begin{array}{l}+0.02 \\
-0.02\end{array}\right)$ & & $0.12\left(\begin{array}{l}+0.02 \\
-0.02\end{array}\right)$ & & \\
\hline & $17^{+}$ & $3871.0^{*}$ & 4038.4 & $1.98\left(\begin{array}{l}+1.11 \\
-0.81\end{array}\right)$ & $>1.01$ & $0.35\left(\begin{array}{l}+0.20 \\
-0.14\end{array}\right)$ & $>0.25$ & \\
\hline & $18^{+}$ & 4320.8 & & $>0.39$ & & $>0.12$ & & \\
\hline & $19^{+}$ & 4859.0 & 5105.1 & & & & & \\
\hline & $20^{+}$ & 5338.4 & & & & & & \\
\hline & $21^{+}$ & 5899.3 & & & & & & \\
\hline & $22^{+}$ & 6450.3 & & & & & & \\
\hline & $23^{+}$ & 6930.6 & & & & & & \\
\hline & $24^{+}$ & 7487.5 & & & & & & \\
\hline & $26^{+}$ & 8622.0 & & & & & & \\
\hline \multirow[t]{8}{*}{${ }_{59}^{134} \operatorname{Pr}_{75}$} & $9^{+}$ & 559 & 797 & & & & & {$[26,86,100,129,134-137]$} \\
\hline & $10^{+}$ & 764 & 931 & & & & & \\
\hline & $11^{+}$ & 930 & 1142 & & & & & \\
\hline & $12^{+}$ & 1213 & 1409 & & & & & \\
\hline & $13^{+}$ & $1537^{*}$ & 1659 & $0.804\left(\begin{array}{l}+0.090 \\
-0.090\end{array}\right)$ & & & & \\
\hline & $14^{+}$ & 1874 & 2017 & $0.678\left({ }_{-0.100}^{+0.100}\right)$ & & $0.039\left({ }_{-0.019}^{+0.019}\right)$ & & \\
\hline & $15^{+}$ & $2243^{*}$ & 2456 & $0.472\left(\begin{array}{l}+0.090 \\
-0.090\end{array}\right)$ & & $0.089\left(\begin{array}{l}+0.020 \\
-0.020\end{array}\right)$ & & \\
\hline & $16^{+}$ & 2643 & 2901 & $0.333\left({ }_{-0.075}^{+0.075}\right)$ & & $0.050\left({ }_{-0.018}^{+0.018}\right)$ & & \\
\hline
\end{tabular}


Table 2 (continued)

\begin{tabular}{|c|c|c|c|c|c|c|c|c|}
\hline \multirow{2}{*}{ Nuclei } & \multirow{2}{*}{$I^{\pi}(\hbar)$} & \multicolumn{2}{|c|}{$E(\mathrm{keV})$} & \multicolumn{2}{|c|}{$B(M 1)\left(\mu_{N}^{2}\right)$} & \multicolumn{2}{|c|}{$B(E 2)\left(\mathrm{e}^{2} \mathrm{~b}^{2}\right)$} & \multirow{2}{*}{ References } \\
\hline & & yrast & side & yrast & side & yrast & side & \\
\hline & $17^{+}$ & 3115 & 3347 & $0.545\left({ }_{-0.067}^{+0.067}\right)$ & & $0.047\left({ }_{-0.017}^{+0.017}\right)$ & & \\
\hline & $18^{+}$ & 3582 & 3858 & & & & & \\
\hline & $19^{+}$ & 4172 & 4338 & & & & & \\
\hline & $20^{+}$ & 4648 & & & & & & \\
\hline & $21^{+}$ & 5343 & & & & & & \\
\hline & $22^{+}$ & 5839 & & & & & & \\
\hline & $23^{+}$ & 6534 & & & & & & \\
\hline \multirow{12}{*}{${ }_{60}^{135} \mathrm{Nd}_{75}$} & $11.5^{-}$ & 2819.4 & & & & & & {$[38,138]$} \\
\hline & $12.5^{-}$ & 2940.4 & & & & & & \\
\hline & $13.5^{-}$ & 3110.5 & 3607.4 & & & & & \\
\hline & $14.5^{-}$ & $3358.2^{*}$ & 3780.4 & $3.2\left(\begin{array}{c}+0.2 \\
-0.2\end{array}\right)$ & & $0.32\left(\begin{array}{l}+0.02 \\
-0.02\end{array}\right)$ & & \\
\hline & $15.5^{-}$ & $3649.5^{*}$ & 4006.4 & $2.5\left(\begin{array}{l}+0.3 \\
-0.3\end{array}\right)$ & $2.7\left(\begin{array}{l}+0.3 \\
-0.3\end{array}\right)$ & $0.32\left({ }_{-0.03}^{+0.03}\right)$ & $0.28\left({ }_{-0.03}^{+0.03}\right)$ & \\
\hline & $16.5^{-}$ & $4007.7^{*}$ & 4288.4 & $2.2\left(\begin{array}{l}+0.2 \\
-0.2\end{array}\right)$ & $2.1\left(\begin{array}{l}+0.2 \\
-0.2\end{array}\right)$ & $0.32\left({ }_{-0.03}^{+0.03}\right)$ & $0.28\left({ }_{-0.03}^{+0.03}\right)$ & \\
\hline & $17.5^{-}$ & $4413.7^{*}$ & 4597.4 & $2.4\left(\begin{array}{l}+0.3 \\
-0.3\end{array}\right)$ & $2.2\left(\begin{array}{l}+0.2 \\
-0.2\end{array}\right)$ & $0.32\left(\begin{array}{l}+0.03 \\
-0.03\end{array}\right)$ & $0.28\left(\begin{array}{c}+0.04 \\
-0.04\end{array}\right)$ & \\
\hline & $18.5^{-}$ & $4852.7^{*}$ & 4969.4 & $1.7\left({ }_{-0.3}^{+0.3}\right)$ & $1.7\left(\begin{array}{l}+0.2 \\
-0.2\end{array}\right)$ & $0.32\left({ }_{-0.04}^{+0.04}\right)$ & $0.29\left({ }_{-0.04}^{+0.04}\right)$ & \\
\hline & $19.5^{-}$ & $5315.7^{*}$ & 5409.4 & $2.1\left({ }_{-0.3}^{+0.3}\right)$ & $1.9\left(\begin{array}{l}+0.3 \\
-0.3\end{array}\right)$ & $0.13\left(\begin{array}{l}+0.03 \\
-0.03\end{array}\right)$ & $0.11\left(\begin{array}{l}+0.03 \\
-0.03\end{array}\right)$ & \\
\hline & $20.5^{-}$ & $5787.7^{*}$ & 5921.4 & $2.1\left({ }_{-0.3}^{+0.3}\right)$ & & $0.19\left(\begin{array}{l}+0.03 \\
-0.03\end{array}\right)$ & & \\
\hline & $21.5^{-}$ & $6281.7^{*}$ & & $2.0\left(\begin{array}{l}+0.3 \\
-0.3\end{array}\right)$ & & $0.21\left(\begin{array}{l}+0.04 \\
-0.04\end{array}\right)$ & & \\
\hline & $22.5^{-}$ & 6799.7 & & & & & & \\
\hline \multirow[t]{15}{*}{${ }_{81}^{194} \mathrm{Tl}_{113}$} & $8^{-}$ & 293 & & & & & & {$[108,143,144]$} \\
\hline & $9^{-}$ & 338 & & & & & & \\
\hline & $10^{-}$ & 434 & & & & & & \\
\hline & $11^{-}$ & 712 & 1175 & & & & & \\
\hline & $12^{-}$ & 957 & 1481 & & & & & \\
\hline & $13^{-}$ & 1361 & 1738 & & & & & \\
\hline & $14^{-}$ & 1644 & 2001 & & & & & \\
\hline & $15^{-}$ & 2112 & 2344 & & & & & \\
\hline & $16^{-}$ & 2404 & 2682 & & & & & \\
\hline & $17^{-}$ & 2882 & 3003 & & & & & \\
\hline & $18^{-}$ & 3130 & 3257 & & & & & \\
\hline & $19^{-}$ & 3381 & 3427 & & $<1.25$ & & & \\
\hline & $20^{-}$ & 3518 & 3628 & & $<1.61$ & & & \\
\hline & $21^{-}$ & 3840 & 3877 & $0.95\left(\begin{array}{c}+0.32 \\
-0.21\end{array}\right)$ & $1.31\left(\begin{array}{c}+0.54 \\
-0.30\end{array}\right)$ & $0.21\left(\begin{array}{l}+0.12 \\
-0.07\end{array}\right)$ & & \\
\hline & $22^{-}$ & 4081 & 4181 & $0.97\left(\begin{array}{l}+0.39 \\
-0.21\end{array}\right)$ & $1.24\left(\begin{array}{l}+0.43 \\
-0.29\end{array}\right)$ & $0.39\left(\begin{array}{l}+0.15 \\
-0.09\end{array}\right)$ & $0.20\left(\begin{array}{l}+0.15 \\
-0.06\end{array}\right)$ & \\
\hline
\end{tabular}


Table 2 (continued)

\begin{tabular}{|c|c|c|c|c|c|c|c|c|}
\hline \multirow{2}{*}{ Nuclei } & \multirow{2}{*}{$I^{\pi}(\hbar)$} & \multicolumn{2}{|c|}{$E(\mathrm{keV})$} & \multicolumn{2}{|c|}{$B(M 1)\left(\mu_{N}^{2}\right)$} & \multicolumn{2}{|c|}{$B(E 2)\left(\mathrm{e}^{2} \mathrm{~b}^{2}\right)$} & \multirow{2}{*}{ References } \\
\hline & & yrast & side & yrast & side & yrast & side & \\
\hline & $23^{-}$ & 4462 & 4560 & $0.34\left(\begin{array}{l}+0.13 \\
-0.07\end{array}\right)$ & $<0.27$ & $0.05\left(\begin{array}{c}+0.03 \\
-0.01\end{array}\right)$ & $<0.07$ & \\
\hline & $24^{-}$ & 4824 & & $0.27\left(\begin{array}{l}+0.18 \\
-0.09\end{array}\right)$ & & $0.07\left({ }_{-0.03}^{+0.05}\right)$ & & \\
\hline
\end{tabular}

